\title{
Connection Formulae for Asymptotics of Solutions of the Degenerate Third Painlevé Equation: II
}

\author{
A. V. Kitaev* \\ Steklov Mathematical Institute \\ Fontanka 27 \\ St. Petersburg 191023 \\ Russia
}

\author{
A. Vartanian \\ Department of Mathematics \\ College of Charleston \\ Charleston, South Carolina 29424 \\ U. S. A.
}

May 25, 2022

\begin{abstract}
The degenerate third Painlevé equation, $u^{\prime \prime}(\tau)=\frac{\left(u^{\prime}(\tau)\right)^{2}}{u(\tau)}-\frac{u^{\prime}(\tau)}{\tau}+\frac{1}{\tau}\left(-8 \epsilon u^{2}(\tau)+2 a b\right)+\frac{b^{2}}{u(\tau)}$, where $\epsilon= \pm 1, b \in \mathbb{R} \backslash\{0\}$, and $a \in \mathbb{C}$, is studied via the Isomonodromy Deformation Method. Asymptotics of general regular and singular solutions as $\tau \rightarrow \pm \infty$ and $\tau \rightarrow \pm \mathrm{i} \infty$ are derived and parametrized in terms of the monodromy data of the associated $2 \times 2$ linear auxiliary problem introduced in [1]. Using these results, three-real-parameter families of solutions that have infinite sequences of zeroes and poles that are asymptotically located along the real and imaginary axes are distinguished: asymptotics of these zeroes and poles are also obtained.
\end{abstract}

2000 Mathematics Subject Classification. 33E17, 34M40, 34M50, 34M55, 34M60

Abbreviated Title. Degenerate Third Painlevé Equation: II

Key Words. Asymptotics, Painlevé transcendents, isomonodromy deformations, WKB method, Stokes phenomena

*E-mail: kitaev@pdmi.ras.ru 


\section{Introduction and the Manifold of Monodromy Data}

In this paper we continue our study [1] of the degenerate third Painlevé equation,

$$
u^{\prime \prime}(\tau)=\frac{\left(u^{\prime}(\tau)\right)^{2}}{u(\tau)}-\frac{u^{\prime}(\tau)}{\tau}+\frac{1}{\tau}\left(-8 \epsilon u^{2}(\tau)+2 a b\right)+\frac{b^{2}}{u(\tau)}, \quad \epsilon= \pm 1,
$$

where the prime denotes differentiation with respect to $\tau$, and $b \in \mathbb{R} \backslash\{0\}$ and $a \in \mathbb{C}$ are parameters. For other Painlevé equations, there exist families of solutions that have zeroes and poles accumulating at the point at infinity (see, for example, [2]). In Part I [1], such solutions were not distinguished; therefore, the main purpose of this work (Part II) is to determine whether or not such solutions exist, and if so, to find their asymptotics.

As shown in [1], Equation (1.1) can be presented as a Hamiltonian system, with the Hamiltonian function, $\mathcal{H}(\tau)$, given by

$$
\mathcal{H}(\tau):=\left(a-\frac{\mathrm{i}}{2}\right) \frac{b}{u(\tau)}+\frac{1}{2 \tau}\left(a-\frac{\mathrm{i}}{2}\right)^{2}+\frac{\tau}{4 u^{2}(\tau)}\left(\left(u^{\prime}(\tau)\right)^{2}+b^{2}\right)+4 \epsilon u(\tau)
$$

In [1] we introduced the auxiliary function

$$
f(\tau):=\frac{\tau\left(u^{\prime}(\tau)-\mathrm{i} b\right)}{4 u(\tau)}-\frac{1}{2}\left(\mathrm{i} a+\frac{1}{2}\right)
$$

which defines Bäcklund transformations for Equation (1.1):

$$
\frac{\mathrm{d}(\tau \mathcal{H}(\tau))}{\mathrm{d} \tau}=4 \epsilon q(\tau)+\mathrm{i} b p(\tau), \quad 2 f(\tau)=q(\tau) p(\tau), \quad-\tau^{2} \frac{\mathrm{d} \mathcal{H}(\tau)}{\mathrm{d} \tau}=\left(2 f(\tau)+\mathrm{i} a+\frac{1}{2}\right)^{2}-\frac{1}{2}\left(\mathrm{i} a+\frac{1}{2}\right)^{2},
$$

where $q(\tau)=u(\tau)$ and $p(\tau)$ is a Bäcklund transformation of $u(\tau)$. In the Hamiltonian setting, the functions $q(\tau)$ and $p(\tau)$ are, respectively, the generalized coordinate and impulse. (More detailed information about the functions $\mathcal{H}(\tau)$ and $f(\tau)$, in particular, the corresponding ODEs they satisfy, can be found in Proposition 1.3 and Remark 1.3 of [1.) In this work, these functions play an important role in the study of the zeroes and poles of $u(\tau)$.

Section 1 of [1] contains a review of the literature on the theory and applications of Equation (1.1); so here we mention only those papers that are related to Equation (1.1) and which have appeared since the publication of [1]. According to the algebro-geometric classification scheme given in [3], the space of initial conditions of Equation (1.1) can be characterized by the extended Dynkin diagram of type $D_{7}$. There is another case of the degenerate third Painlevé equation which can be obtained by a similarity reduction of the well-known Sine-Gordon equation: in the classification scheme of [3], this equation is of type $D_{8}$. The latter equation is known to be equivalent, via a quadratic transformation, to a special case of the "complete" third Painlevé equation (type $D_{6}$ in the classification of [3); therefore, we use the term "degenerate" to specify only Equation (1.1), or its equivalent forms. Asymptotics of solutions of the $D_{8}$ equation were studied via the Isomonodromy Deformation Method in 2. Recently, asymptotics of the so-called instanton solutions of the $D_{8}$ equation were obtained in [4 via the exact WKB analysis. We also mention the recent work [5], where a class of semi-flat Calabi-Yau metrics is obtained in terms of real solutions of Equation (1.1) with $a=0$.

In this work we apply the Isomonodromy Deformation Method: the reader can find some basic ideas and references concerning this method in Sections 1 and 2 of [1]. We also mention the new monograph [6], which reflects some recent developments of this method and of a closely related technique based on a steepest-descent-type analysis of the associated Riemann-Hilbert problem [7. Although Equation (1.1) resembles one of the standard representatives of the list of the Painlevé equations, its asymptotic study via the Isomonodromy Deformation Method contains, in contrast to the other Painlevé equations, additional technical difficulties: the problem is that the corresponding FuchsGarnier (or Lax) pair is degenerate (see 1 for details); therefore, in contrast to the non-degenerate versions of the Painlevé equations, its associated WKB analysis requires a much more careful evaluation of the correction terms. In fact, this is one of the reasons why the present work took 6 years to complete since the appearance of [1].

In order to make the presentation as self-contained as possible, we now embark on succinctly reminding the reader about some of the basic facts introduced in Sections 1 and 2 of [1]. 
Our study of Equation (1.1) is based on the following Fuchs-Garnier (or Lax) pair (see Proposition 2.1 of [1]):

$$
\partial_{\mu} \Psi(\mu, \tau)=\widetilde{\mathcal{U}}(\mu, \tau) \Psi(\mu, \tau), \quad \partial_{\tau} \Psi(\mu, \tau)=\widetilde{\mathcal{V}}(\mu, \tau) \Psi(\mu, \tau),
$$

where

$$
\begin{aligned}
\tilde{U}(\mu, \tau) & =-2 \mathrm{i} \tau \mu \sigma_{3}+2 \tau\left(\begin{array}{cc}
0 & \frac{2 \mathrm{i} A(\tau)}{\sqrt{-A(\tau) B(\tau)}} \\
-D(\tau) & 0
\end{array}\right)-\frac{1}{\mu}\left(\mathrm{i} a+\frac{2 \tau A(\tau) D(\tau)}{\sqrt{-A(\tau) B(\tau)}}+\frac{1}{2}\right) \sigma_{3} \\
& +\frac{1}{\mu^{2}}\left(\begin{array}{cc}
0 & \widetilde{\alpha}(\tau) \\
\mathrm{i} \tau B(\tau) & 0
\end{array}\right), \\
\widetilde{\mathcal{V}}(\mu, \tau) & =-\mathrm{i} \mu^{2} \sigma_{3}+\mu\left(\begin{array}{c}
0 \\
-D(\tau)
\end{array}\right. \\
& -\frac{1}{2 \tau \mu}\left(\begin{array}{cc}
0 & \widetilde{\alpha}(\tau) \\
\mathrm{i} \tau B(\tau) & 0
\end{array}\right),
\end{aligned}
$$

with $\sigma_{3}=\left(\begin{array}{cc}1 & 0 \\ 0 & -1\end{array}\right)$, and

$$
\widetilde{\alpha}(\tau):=-\frac{2}{B(\tau)}(\mathrm{i} a \sqrt{-A(\tau) B(\tau)}+\tau(A(\tau) D(\tau)+B(\tau) C(\tau)))
$$

Proposition 1.1. The Frobenius compatibility condition of System (1.4) for arbitrary values of $\mu \in \mathbb{C}$ and for differentiable, scalar-valued functions $A(\tau), B(\tau), C(\tau)$, and $D(\tau)$ is that these functions satisfy the following system of isomonodromy deformations:

$$
\begin{array}{cc}
A^{\prime}(\tau)=4 C(\tau) \sqrt{-A(\tau) B(\tau)}, & B^{\prime}(\tau)=-4 D(\tau) \sqrt{-A(\tau) B(\tau)}, \\
(\tau C(\tau))^{\prime}=2 \mathrm{i} a C(\tau)-2 \tau A(\tau), & (\tau D(\tau))^{\prime}=-2 \mathrm{i} a D(\tau)+2 \tau B(\tau), \\
(\sqrt{-A(\tau) B(\tau)})^{\prime}=2(A(\tau) D(\tau)-B(\tau) C(\tau)) .
\end{array}
$$

Remark 1.1. Hereafter, all explicit $\tau$ dependencies are suppressed, except where confusion may arise.

A relation between the Fuchs-Garnier pair (1.4) and the degenerate third Painlevé equation (1.1) is given by

Proposition $1.2([1])$. Let $u=u(\tau)$ and $\varphi=\varphi(\tau)$ solve the system

$$
\begin{gathered}
u^{\prime \prime}=\frac{\left(u^{\prime}\right)^{2}}{u}-\frac{u^{\prime}}{\tau}+\frac{1}{\tau}\left(-8 \epsilon u^{2}+2 a b\right)+\frac{b^{2}}{u}, \\
\varphi^{\prime}=\frac{2 a}{\tau}+\frac{b}{u},
\end{gathered}
$$

where $\epsilon= \pm 1$, and $a, b \in \mathbb{C}$ are independent of $\tau$. Then,

$$
A(\tau):=\frac{u(\tau)}{\tau} \mathrm{e}^{\mathrm{i} \varphi(\tau)}, \quad B(\tau):=-\frac{u(\tau)}{\tau} \mathrm{e}^{-\mathrm{i} \varphi(\tau)}, \quad C(\tau):=\frac{\epsilon \tau A^{\prime}(\tau)}{4 u(\tau)}, \quad D(\tau):=-\frac{\epsilon \tau B^{\prime}(\tau)}{4 u(\tau)}
$$

solve System (1.5). Conversely, let $A(\tau) \not \equiv 0, B(\tau) \not \equiv 0, C(\tau)$, and $D(\tau)$ solve System (1.5), and define

$$
u(\tau):=\epsilon \tau \sqrt{-A(\tau) B(\tau)}, \quad \varphi(\tau):=-\frac{\mathrm{i}}{2} \ln \left(-\frac{A(\tau)}{B(\tau)}\right), \quad \text { and } \quad b:=u(\tau)\left(\varphi^{\prime}(\tau)-\frac{2 a}{\tau}\right) .
$$

Then $b$ is independent of $\tau$, and $u(\tau)$ and $\varphi(\tau)$ solve System (1.6).

In this work asymptotics (as $\tau \rightarrow \pm \infty$ and $\tau \rightarrow \pm \mathrm{i} \infty$ ) of solutions of Equation (1.1) are parametrized in terms of the monodromy data of System (1.4). This parametrization is equivalent to finding the corresponding connection formulae; indeed, given asymptotics of some solution as $\tau \rightarrow+\infty$, say, one uses it to determine the corresponding monodromy data, and therefore to obtain asymptotics of the same solution as $\tau \rightarrow-\infty$ or $\tau \rightarrow \pm \mathrm{i} \infty$. Furthermore, employing results from 11, one arrives at asymptotics of the same solution as $\tau \rightarrow \pm 0$ or $\tau \rightarrow \pm \mathrm{i} 0$. Therefore, it is important to remind the reader about the definition of the monodromy data of System (1.4) given in [1]. 
On the complex $\mu$-plane, System (1.4) has two irregular singular points, $\mu=\infty$ and $\mu=0$. For $\delta_{\infty}, \delta_{0}>0$ and $k \in \mathbb{Z}$, define the (sectorial) neighborhoods $\Omega_{k}^{\infty}$ and $\Omega_{k}^{0}$, respectively, of these (singular) points:

$$
\begin{aligned}
\Omega_{k}^{\infty} & :=\left\{\mu \in \mathbb{C}:|\mu|>\delta_{\infty}^{-1},-\frac{\pi}{2}+\frac{\pi k}{2}<\arg \mu+\frac{1}{2} \arg \tau<\frac{\pi}{2}+\frac{\pi k}{2}\right\}, \\
\Omega_{k}^{0} & :=\left\{\mu \in \mathbb{C}:|\mu|<\delta_{0},-\pi+\pi k<\arg \mu-\frac{1}{2} \arg \tau-\frac{1}{2} \arg (\epsilon b)<\pi+\pi k\right\} .
\end{aligned}
$$

The following Proposition is a direct consequence of general asymptotic results for linear ODEs $[8,9$.

Proposition 1.3 ([1]). For $k \in \mathbb{Z}$, there exist solutions $Y_{k}^{\infty}(\mu)=Y_{k}^{\infty}(\mu, \tau)$ and $X_{k}^{0}(\mu)=X_{k}^{0}(\mu, \tau)$ of System (1.4) which are uniquely defined by the following asymptotic expansions:

$$
\begin{aligned}
Y_{k}^{\infty}(\mu) \underset{\substack{\mu \rightarrow \infty \\
\mu \in \Omega_{k}^{\infty}}}{:=}\left(\mathrm{I}+\frac{1}{\mu} \Psi^{(1)}+\frac{1}{\mu^{2}} \Psi^{(2)}+\cdots\right) \exp \left(-\mathrm{i}\left(\tau \mu^{2}+\left(a-\frac{\mathrm{i}}{2}\right) \ln \mu\right) \sigma_{3}\right), \\
X_{k}^{0}(\mu) \underset{\substack{:=\\
\mu \in 0 \\
\mu \in \Omega_{k}^{0}}}{ } \Psi_{0}\left(\mathrm{I}+\mathcal{Z}_{1} \mu+\cdots\right) \exp \left(-\frac{\mathrm{i} \sqrt{\tau \epsilon b}}{\mu} \sigma_{3}\right),
\end{aligned}
$$

where $\ln \mu:=\ln |\mu|+\mathrm{i} \arg \mu$,

$$
\begin{aligned}
& \Psi^{(1)}=\left(\begin{array}{cc}
0 & \frac{A}{\sqrt{-A B}} \\
\frac{D}{2 \mathrm{i}} & 0
\end{array}\right), \quad \Psi^{(2)}=\left(\begin{array}{cc}
\psi_{11}^{(2)} & 0 \\
0 & \psi_{22}^{(2)}
\end{array}\right), \\
& \psi_{11}^{(2)}=-\frac{\mathrm{i}}{2}\left(\tau \sqrt{-A B}+\tau D C+\frac{A D}{\sqrt{-A B}}\right), \quad \psi_{22}^{(2)}=\frac{\mathrm{i} \tau}{2}(\sqrt{-A B}+C D), \\
& \Psi_{0}=\frac{\mathrm{i}}{\sqrt{2}}\left(\frac{(\epsilon b)^{1 / 4}}{\tau^{1 / 4} \sqrt{B}}\right)^{\sigma_{3}}\left(\sigma_{1}+\sigma_{3}\right), \quad \mathcal{Z}_{1}=\left(\begin{array}{cc}
z_{1}^{(11)} & z_{1}^{(12)} \\
-z_{1}^{(12)} & -z_{1}^{(11)}
\end{array}\right), \\
& z_{1}^{(11)}=\frac{\left(\mathrm{i} a+\frac{1}{2}+\frac{2 \tau A D}{\sqrt{-A B}}\right)^{2}}{2 \mathrm{i} \sqrt{\tau \epsilon b}}-\frac{2 \mathrm{i} \tau^{3 / 2} \sqrt{-A B}}{\sqrt{\epsilon b}}-\frac{D \sqrt{\tau \epsilon b}}{B}, \quad z_{1}^{(12)}=\frac{\left(\mathrm{i} a+\frac{1}{2}+\frac{2 \tau A D}{\sqrt{-A B}}\right)}{2 \mathrm{i} \sqrt{\tau \epsilon b}},
\end{aligned}
$$

with $\mathrm{I}=\left(\begin{array}{ll}1 & 0 \\ 0 & 1\end{array}\right)$, and $\sigma_{1}=\left(\begin{array}{ll}0 & 1 \\ 1 & 0\end{array}\right)$.

Remark 1.2. The canonical solutions $X_{k}^{0}(\mu)$ are defined uniquely, provided the branch of $\sqrt{B(\tau)}$ is fixed. Hereafter, the branch of $\sqrt{B(\tau)}$ is not fixed; therefore, the set of canonical solutions $\left\{X_{k}^{0}(\mu)\right\}_{k \in \mathbb{Z}}$ is defined up to a sign. This ambiguity doesn't affect the definition of the Stokes multipliers (see Equations (1.8) below), but results in an ambiguity of sign in the definition of the connection matrix, $G$ (see Equation (1.11) below).

The canonical solutions, $Y_{k}^{\infty}(\mu)$ and $X_{k}^{0}(\mu)$, enable one to define the Stokes matrices, $S_{k}^{\infty}$ and $S_{k}^{0}$ :

$$
Y_{k+1}^{\infty}(\mu)=Y_{k}^{\infty}(\mu) S_{k}^{\infty}, \quad X_{k+1}^{0}(\mu)=X_{k}^{0}(\mu) S_{k}^{0} .
$$

The Stokes matrices are independent of the parameters $\mu$ and $\tau$, and have the following structures:

$$
S_{2 k}^{\infty}=\left(\begin{array}{cc}
1 & 0 \\
s_{2 k}^{\infty} & 1
\end{array}\right), \quad S_{2 k+1}^{\infty}=\left(\begin{array}{cc}
1 & s_{2 k+1}^{\infty} \\
0 & 1
\end{array}\right), \quad S_{2 k}^{0}=\left(\begin{array}{cc}
1 & s_{2 k}^{0} \\
0 & 1
\end{array}\right), \quad S_{2 k+1}^{0}=\left(\begin{array}{cc}
1 & 0 \\
s_{2 k+1}^{0} & 1
\end{array}\right) .
$$

The parameters $s_{n}^{\infty}$ and $s_{n}^{0}, n \in \mathbb{Z}$, are called the Stokes multipliers. One can show that

$$
S_{k+4}^{\infty}=\mathrm{e}^{-2 \pi\left(a-\frac{\mathrm{i}}{2}\right) \sigma_{3}} S_{k}^{\infty} \mathrm{e}^{2 \pi\left(a-\frac{\mathrm{i}}{2}\right) \sigma_{3}}, \quad S_{k+2}^{0}=S_{k}^{0} .
$$

Equations (1.9) show that the number of independent Stokes multipliers does not exceed six; for example, $s_{0}^{0}, s_{1}^{0}, s_{0}^{\infty}, s_{1}^{\infty}, s_{2}^{\infty}$, and $s_{3}^{\infty}$. Furthermore, due to the special structure of System (1.4), that is, the coefficient matrices of odd (resp., even) powers of $\mu$ in $\widetilde{\mathcal{U}}(\mu, \tau)$ are diagonal (resp., off-diagonal) and vice-versa for $\widetilde{\mathcal{V}}(\mu, \tau)$, one can deduce the following relations for the Stokes matrices (multipliers):

$$
S_{k+2}^{\infty}=\sigma_{3} \mathrm{e}^{-\pi\left(a-\frac{\mathrm{i}}{2}\right) \sigma_{3}} S_{k}^{\infty} \mathrm{e}^{\pi\left(a-\frac{\mathrm{i}}{2}\right) \sigma_{3}} \sigma_{3}, \quad S_{k}^{0}=\sigma_{1} S_{k+1}^{0} \sigma_{1} .
$$


Equations (1.10) reduce the number of independent Stokes multipliers by a factor of 2, that is, all Stokes multipliers can be expressed in terms of $s_{0}^{0}, s_{0}^{\infty}, s_{1}^{\infty}$, and the parameter of formal monodromy, $a$. There is one more relation between the Stokes multipliers, which follows from the so-called cyclic relation (see below). Define the monodromy matrices at infinity, $M^{\infty}$, and at zero, $M^{0}$, by the following relations:

$$
Y_{0}^{\infty}\left(\mu \mathrm{e}^{-2 \pi \mathrm{i}}\right):=Y_{0}^{\infty}(\mu) M^{\infty}, \quad X_{0}^{0}\left(\mu \mathrm{e}^{-2 \pi \mathrm{i}}\right):=X_{0}^{0}(\mu) M^{0} .
$$

Since $Y_{0}^{\infty}(\mu)$ and $X_{0}^{0}(\mu)$ are solutions of System (1.4), they differ by a right-hand (matrix) factor $G$ :

$$
Y_{0}^{\infty}(\mu):=X_{0}^{0}(\mu) G,
$$

where $G$ is called the connection matrix. As matrices relating fundamental solutions of System (1.4), the monodromy $y_{2}$ connection, and Stokes matrices are independent of $\mu$ and $\tau$; furthermore, since $\operatorname{tr}(\widetilde{\mathcal{U}}(\mu, \tau))=\operatorname{tr}(\widetilde{\mathcal{V}}(\mu, \tau))=0$, it follows that

$$
\operatorname{det}\left(M^{0}\right)=\operatorname{det}\left(M^{\infty}\right)=\operatorname{det}(G)=1 .
$$

From the definition of the monodromy and connection matrices, one deduces the following cyclic relation:

$$
G M^{\infty}=M^{0} G .
$$

The monodromy matrices can be expressed in terms of the Stokes matrices:

$$
M^{\infty}=S_{0}^{\infty} S_{1}^{\infty} S_{2}^{\infty} S_{3}^{\infty} \mathrm{e}^{-2 \pi\left(a-\frac{\mathrm{i}}{2}\right) \sigma_{3}}, \quad M^{0}=S_{0}^{0} S_{1}^{0} .
$$

The Stokes multipliers, $s_{0}^{0}, s_{0}^{\infty}$, and $s_{1}^{\infty}$, the elements of the connection matrix, $(G)_{i j}=: g_{i j}, i, j=1,2$, and the parameter of formal monodromy, $a$, are called the monodromy data. Consider $\mathbb{C}^{8}$ with coordinates $\left(a, s_{0}^{0}, s_{0}^{\infty}, s_{1}^{\infty}, g_{11}, g_{12}, g_{21}, g_{22}\right)$. The algebraic variety $\operatorname{defined}$ by $\operatorname{det}(G)=1$ and the $s e m i$ cyclic relation

$$
G^{-1} S_{0}^{0} \sigma_{1} G=S_{0}^{\infty} S_{1}^{\infty} \sigma_{3} \mathrm{e}^{-\pi\left(a-\frac{\mathrm{i}}{2}\right) \sigma_{3}}
$$

is called the manifold of monodromy data, $\mathcal{M}$. Since only three of the four equations in the semi-cyclic relation are independent, it is clear that $\operatorname{dim}_{\mathbb{C}}(\mathcal{M})=4$; more precisely, the equations defining $\mathcal{M}$ are:

$$
\begin{gathered}
s_{0}^{\infty} s_{1}^{\infty}=-1-\mathrm{e}^{-2 \pi a}-\mathrm{i} s_{0}^{0} \mathrm{e}^{-\pi a}, \quad g_{22} g_{21}-g_{11} g_{12}+s_{0}^{0} g_{11} g_{22}=\mathrm{ie} \mathrm{e}^{-\pi a}, \\
g_{11}^{2}-g_{21}^{2}-s_{0}^{0} g_{11} g_{21}=\mathrm{i} s_{0}^{\infty} \mathrm{e}^{-\pi a}, \quad g_{22}^{2}-g_{12}^{2}+s_{0}^{0} g_{12} g_{22}=\mathrm{i} s_{1}^{\infty} \mathrm{e}^{\pi a}, \quad g_{11} g_{22}-g_{12} g_{21}=1 .
\end{gathered}
$$

Remark 1.3. To achieve a one-to-one correspondence between the coefficients of System (1.4) and the points on $\mathcal{M}$, one has to factorize $\mathcal{M}$ by the involution $G \rightarrow-G$ (cf. Remark 1.2).

We now describe the contents of this paper.

In Section 2 the main asymptotic results for $u(\tau), \mathcal{H}(\tau)$, and $f(\tau)$ as $\tau \rightarrow \pm \infty$ are presented. Solutions $u(\tau)$ having infinite sequences of zeroes and poles accumulating at the point at infinity of the real and imaginary axes are also specified, and the asymptotic distribution of these sequences are obtained.

In Section 3 (rather technical in nature) the asymptotic solution of the direct monodromy problem for the $\mu$-part of System (1.4), under certain restrictions on its coefficients, is presented for positive real $\tau$. This asymptotic solution is based on matching WKB-asymptotics of the fundamental solution of System (1.4) with its approximation in terms of parabolic-cylinder functions near the double-turning point.

In Section 4 the results of Section 3 are inverted in order to solve the inverse monodromy problem for the $\mu$-part of System (1.4). At this stage, explicit asymptotics for the coefficients of the $\mu$-part of System (1.4) are parametrized in terms of the monodromy data. Under the assumption that the monodromy data are constant and satisfy certain conditions, one finds that the asymptotics obtained satisfy all of the conditions that were imposed in Section 3. According to the justification scheme presented in [10, it follows that there exists exact isomonodromy deformations corresponding to these monodromy data, that is, solutions of the system of isomonodromy deformations (1.5), whose asymptotics coincide with the ones obtained in this section.

Appendix A contains the Laurent expansions at zeroes and poles of the function $u(\tau)$ together with the corresponding expansions of the associated functions $\mathcal{H}(\tau)$ and $f(\tau)$. These results are used in 
Section 4 to complete the proof of the asymptotic distribution of zeroes and poles of $u(\tau)$ for positive real $\tau$.

In Appendix B the asymptotic results for $\tau \rightarrow \infty$ obtained in this paper are compared with the corresponding asymptotitcs in [1, and misprints from [1] are corrected. This comparison is used in Section 4 to resolve a sign ambiguity in the solution of the inverse monodromy problem.

The main body of this paper is devoted to asymptotics as $\tau \rightarrow+\infty$. To extend the results to negative and pure imaginary $\tau$, one applies the action of the Lie-point symmetries $\tau \rightarrow-\tau$ and $\tau \rightarrow \pm \mathrm{i} \tau$ on the manifold of monodromy data that were derived in Subsection 6.2 of [1. Asymptotics for real $\tau$ are presented in Section 2 (as mentioned above), and asymptotics for imaginary $\tau$ are given in Appendix C.

We plan to devote the third part of our studies of the degenerate third Painlevé Equation (1.1) to the extension of our results to complex values of the parameter $b$, and the discussion of the behavior of some special solutions on the real and imaginary axes together with the comparison of asymptotic and numerical results.

\section{Summary of Results}

In this work the detailed analysis for asymptotics of $u(\tau)$ is presented for the case $\tau \rightarrow+\infty$ and $\epsilon b>0$. The analytic continuation of the function $u(\tau)$ from positive values of the parameters $\tau$ and $\epsilon b$ to negative values of these parameters is not single valued; therefore, in order to reflect this fact, write $\tau=|\tau| \mathrm{e}^{\mathrm{i} \pi \varepsilon_{1}}$ and $\epsilon b=|\epsilon b| \mathrm{e}^{\mathrm{i} \pi \varepsilon_{2}}$, where $\varepsilon_{1}, \varepsilon_{2}=0, \pm 1$. The corresponding asymptotics, for both positive and negative values of these parameters, of $u(\tau)$, and the associated functions $\mathcal{H}(\tau)$ and $f(\tau)$, are convenient to express in terms of the auxiliary mapping $\mathcal{F}_{\varepsilon_{1}, \varepsilon_{2}}$ (see below), which is an isomorphism of the manifold of monodromy data 1 , $\mathcal{M}$. The following definition of $\mathcal{F}_{\varepsilon_{1}, \varepsilon_{2}}$ is based on Section 6 of [1], that is, transformation 6.2 .1 changing $22 \rightarrow-\tau$ and transformation 6.2 .2 changing $3 \rightarrow-a$.

Define $\mathcal{F}_{\varepsilon_{1}, \varepsilon_{2}}:\left(a, s_{0}^{0}, s_{0}^{\infty}, s_{1}^{\infty}, g_{11}, g_{12}, g_{21}, g_{22}\right) \rightarrow\left((-1)^{\varepsilon_{2}} a, s_{0}^{0}, s_{0}^{\infty}\left(\varepsilon_{1}, \varepsilon_{2}\right), s_{1}^{\infty}\left(\varepsilon_{1}, \varepsilon_{2}\right), g_{11}\left(\varepsilon_{1}, \varepsilon_{2}\right)\right.$, $\left.g_{12}\left(\varepsilon_{1}, \varepsilon_{2}\right), g_{21}\left(\varepsilon_{1}, \varepsilon_{2}\right), g_{22}\left(\varepsilon_{1}, \varepsilon_{2}\right)\right), \varepsilon_{1}, \varepsilon_{2}=0, \pm 1$ :

(1) $\mathcal{F}_{0,0}$ is the identity mapping: $s_{0}^{\infty}(0,0)=s_{0}^{\infty}, s_{1}^{\infty}(0,0)=s_{1}^{\infty}$, and $g_{i j}(0,0)=g_{i j}, i, j=1,2$;

(2) $\mathcal{F}_{0,-1}: s_{0}^{\infty}(0,-1)=s_{1}^{\infty} \mathrm{e}^{\pi a}, s_{1}^{\infty}(0,-1)=s_{0}^{\infty} \mathrm{e}^{\pi a}, g_{11}(0,-1)=-g_{22} \mathrm{e}^{\frac{\pi a}{2}}, g_{12}(0,-1)=-\left(g_{21}+\right.$ $\left.s_{0}^{\infty} g_{22}\right) \mathrm{e}^{-\frac{\pi a}{2}}, g_{21}(0,-1)=-\left(g_{12}-s_{0}^{0} g_{22}\right) \mathrm{e}^{\frac{\pi a}{2}}$, and $g_{22}(0,-1)=-\left(g_{11}-s_{0}^{0} g_{21}+\left(g_{12}-s_{0}^{0} g_{22}\right) s_{0}^{\infty}\right) \mathrm{e}^{-\frac{\pi a}{2}}$;

(3) $\mathcal{F}_{0,1}: s_{0}^{\infty}(0,1)=s_{1}^{\infty} \mathrm{e}^{\pi a}, s_{1}^{\infty}(0,1)=s_{0}^{\infty} \mathrm{e}^{\pi a}, g_{11}(0,1)=-\mathrm{i} g_{12} \mathrm{e}^{\frac{\pi a}{2}}, g_{12}(0,1)=-\mathrm{i}\left(g_{11}+s_{0}^{\infty} g_{12}\right) \mathrm{e}^{-\frac{\pi a}{2}}$, $g_{21}(0,1)=-\mathrm{i} g_{22} \mathrm{e}^{\frac{\pi a}{2}}$, and $g_{22}(0,1)=-\mathrm{i}\left(g_{21}+s_{0}^{\infty} g_{22}\right) \mathrm{e}^{-\frac{\pi a}{2}}$;

(4) $\mathcal{F}_{-1,0}: s_{0}^{\infty}(-1,0)=-s_{0}^{\infty} \mathrm{e}^{-\pi a}, s_{1}^{\infty}(-1,0)=-s_{1}^{\infty} \mathrm{e}^{\pi a}, g_{11}(-1,0)=g_{21} \mathrm{e}^{-\frac{\pi a}{2}}, g_{12}(-1,0)=-g_{22} \mathrm{e}^{\frac{\pi a}{2}}$, $g_{21}(-1,0)=\left(g_{11}-s_{0}^{0} g_{21}\right) \mathrm{e}^{-\frac{\pi a}{2}}$, and $g_{22}(-1,0)=-\left(g_{12}-s_{0}^{0} g_{22}\right) \mathrm{e}^{\frac{\pi a}{2}}$;

(5) $\mathcal{F}_{-1,-1}: s_{0}^{\infty}(-1,-1)=-s_{1}^{\infty}, s_{1}^{\infty}(-1,-1)=-s_{0}^{\infty} \mathrm{e}^{2 \pi a}, g_{11}(-1,-1)=g_{12}-s_{0}^{0} g_{22}, g_{12}(-1,-1)=$ $-g_{11}+s_{0}^{0} g_{21}-\left(g_{12}-s_{0}^{0} g_{22}\right) s_{0}^{\infty}, g_{21}(-1,-1)=g_{22}-\left(g_{12}-s_{0}^{0} g_{22}\right) s_{0}^{0}$, and $g_{22}(-1,-1)=-g_{21}+\left(g_{11}-\right.$ $\left.s_{0}^{0} g_{21}\right) s_{0}^{0}-\left(g_{22}-\left(g_{12}-s_{0}^{0} g_{22}\right) s_{0}^{0}\right) s_{0}^{\infty}$;

(6) $\mathcal{F}_{-1,1}: s_{0}^{\infty}(-1,1)=-s_{1}^{\infty}, s_{1}^{\infty}(-1,1)=-s_{0}^{\infty} \mathrm{e}^{2 \pi a}, g_{11}(-1,1)=\mathrm{i} g_{22}, g_{12}(-1,1)=-\mathrm{i}\left(g_{21}+s_{0}^{\infty} g_{22}\right)$, $g_{21}(-1,1)=\mathrm{i}\left(g_{12}-s_{0}^{0} g_{22}\right)$, and $g_{22}(-1,1)=-\mathrm{i}\left(g_{11}-s_{0}^{0} g_{21}+\left(g_{12}-s_{0}^{0} g_{22}\right) s_{0}^{\infty}\right)$;

(7) $\mathcal{F}_{1,0}: s_{0}^{\infty}(1,0)=-s_{0}^{\infty} \mathrm{e}^{\pi a}, s_{1}^{\infty}(1,0)=-s_{1}^{\infty} \mathrm{e}^{-\pi a}, g_{11}(1,0)=\left(g_{21}+s_{0}^{0} g_{11}\right) \mathrm{e}^{\frac{\pi a}{2}}, g_{12}(1,0)=-\left(g_{22}+\right.$ $\left.s_{0}^{0} g_{12}\right) \mathrm{e}^{-\frac{\pi a}{2}}, g_{21}(1,0)=g_{11} \mathrm{e}^{\frac{\pi a}{2}}$, and $g_{22}(1,0)=-g_{12} \mathrm{e}^{-\frac{\pi a}{2}}$

(8) $\mathcal{F}_{1,-1}: s_{0}^{\infty}(1,-1)=-s_{1}^{\infty} \mathrm{e}^{2 \pi a}, s_{1}^{\infty}(1,-1)=-s_{0}^{\infty}, g_{11}(1,-1)=g_{12} \mathrm{e}^{\pi a}, g_{12}(1,-1)=-\left(g_{11}+\right.$ $\left.s_{0}^{\infty} g_{12}\right) \mathrm{e}^{-\pi a}, g_{21}(1,-1)=g_{22} \mathrm{e}^{\pi a}$, and $g_{22}(1,-1)=-\left(g_{21}+s_{0}^{\infty} g_{22}\right) \mathrm{e}^{-\pi a}$;

(9) $\mathcal{F}_{1,1}: s_{0}^{\infty}(1,1)=-s_{1}^{\infty} \mathrm{e}^{2 \pi a}, s_{1}^{\infty}(1,1)=-s_{0}^{\infty}, g_{11}(1,1)=\mathrm{i}\left(g_{22}+s_{0}^{0} g_{12}\right) \mathrm{e}^{\pi a}, g_{12}(1,1)=-\mathrm{i}\left(g_{21}+s_{0}^{0} g_{11}+\right.$ $\left.\left(g_{22}+s_{0}^{0} g_{12}\right) s_{0}^{\infty}\right) \mathrm{e}^{-\pi a}$, and $g_{22}(1,1)=-\mathrm{i}\left(g_{11}+s_{0}^{\infty} g_{12}\right) \mathrm{e}^{-\pi a}$.

\footnotetext{
${ }^{1}$ There is a misprint on page 1173 (Section 3) of [1]: for items (2), (3), (5), (6), (8) and (9) in the definition of the auxiliary mapping $\mathcal{F}_{\varepsilon_{1}, \varepsilon_{2}}$, the change $a \rightarrow-a$ should be made everywhere.

${ }^{2}$ In transformation 6.2.1, $\epsilon b \rightarrow \epsilon b$ and $a \rightarrow a$, that is, $\epsilon_{n} b_{n}=\epsilon_{o} b_{o}$ and $a_{n}=a_{o}$.

${ }^{3}$ In transformation $6.2 .2, \tau \rightarrow \tau$, that is, $\tau_{n}=\tau_{o}$.

${ }^{4} s_{0}^{0}\left(\varepsilon_{1}, \varepsilon_{2}\right)=s_{0}^{0}$.
} 
Remark 2.1. The roots of positive quantities are assumed positive, whilst the branches of the roots of complex quantities can be taken arbitrarily, unless stated otherwise. Furthermore, it is assumed that, for negative real $z$, the following branches are always taken: $z^{1 / 3}:=-|z|^{1 / 3}$ and $z^{2 / 3}:=\left(z^{1 / 3}\right)^{2}$.

Theorem 2.1. Let $\varepsilon_{1}, \varepsilon_{2}=0, \pm 1, \epsilon b=|\epsilon b| \mathrm{e}^{\mathrm{i} \pi \varepsilon_{2}}$, and $u(\tau)$ be a solution of Equation (1.1) corresponding to the monodromy data $\left(a, s_{0}^{0}, s_{0}^{\infty}, s_{1}^{\infty}, g_{11}, g_{12}, g_{21}, g_{22}\right)$. Suppose that

$$
g_{11}\left(\varepsilon_{1}, \varepsilon_{2}\right) g_{12}\left(\varepsilon_{1}, \varepsilon_{2}\right) g_{21}\left(\varepsilon_{1}, \varepsilon_{2}\right) g_{22}\left(\varepsilon_{1}, \varepsilon_{2}\right) \neq 0, \quad \operatorname{Re}\left(\widetilde{\nu}\left(\varepsilon_{1}, \varepsilon_{2}\right)+1\right) \in(0,1) \backslash\left\{\frac{1}{2}\right\}
$$

where

$$
\widetilde{\nu}\left(\varepsilon_{1}, \varepsilon_{2}\right)+1:=\frac{\mathrm{i}}{2 \pi} \ln \left(g_{11}\left(\varepsilon_{1}, \varepsilon_{2}\right) g_{22}\left(\varepsilon_{1}, \varepsilon_{2}\right)\right) .
$$

Then there exist $\delta_{G}$ satisfying, for $0<\operatorname{Re}\left(\widetilde{\nu}\left(\varepsilon_{1}, \varepsilon_{2}\right)+1\right)<\frac{1}{2}$, the inequality

$$
0<\delta_{G}<\frac{1}{3}\left(\frac{1+2 \operatorname{Re}\left(\widetilde{\nu}\left(\varepsilon_{1}, \varepsilon_{2}\right)+1\right)}{7+6 \operatorname{Re}\left(\widetilde{\nu}\left(\varepsilon_{1}, \varepsilon_{2}\right)+1\right)}\right),
$$

and, for $\frac{1}{2}<\operatorname{Re}\left(\widetilde{\nu}\left(\varepsilon_{1}, \varepsilon_{2}\right)+1\right)<1$, the inequality

$$
0<\delta_{G}<\frac{1}{3}\left(\frac{3-2 \operatorname{Re}\left(\widetilde{\nu}\left(\varepsilon_{1}, \varepsilon_{2}\right)+1\right)}{9+2 \operatorname{Re}\left(\widetilde{\nu}\left(\varepsilon_{1}, \varepsilon_{2}\right)+1\right)}\right)
$$

such that $u(\tau)$ has the asymptotic expansion

$$
\begin{aligned}
u(\tau) \underset{\tau \rightarrow \infty \mathrm{e}^{\mathrm{i} \pi \varepsilon_{1}}}{=} \frac{\epsilon(\epsilon b)^{2 / 3}}{2} \tau^{1 / 3}\left(1-\frac{3}{2 \sin ^{2}\left(\frac{1}{2} \vartheta\left(\varepsilon_{1}, \varepsilon_{2}, \tau\right)\right)}\right) \\
\underset{\substack{\tau \rightarrow \infty \mathrm{e}^{\mathrm{i} \pi \varepsilon_{1}}}}{ } \frac{\epsilon(\epsilon b)^{2 / 3}}{2} \tau^{1 / 3} \frac{\sin \left(\frac{1}{2} \vartheta\left(\varepsilon_{1}, \varepsilon_{2}, \tau\right)-\vartheta_{0}\right) \sin \left(\frac{1}{2} \vartheta\left(\varepsilon_{1}, \varepsilon_{2}, \tau\right)+\vartheta_{0}\right)}{\sin ^{2}\left(\frac{1}{2} \vartheta\left(\varepsilon_{1}, \varepsilon_{2}, \tau\right)\right)},
\end{aligned}
$$

where

$$
\begin{aligned}
\vartheta\left(\varepsilon_{1}, \varepsilon_{2}, \tau\right): & \phi(\tau)-\mathrm{i}\left(\left(\widetilde{\nu}\left(\varepsilon_{1}, \varepsilon_{2}\right)+1\right)-\frac{1}{2}\right) \ln \phi(\tau)-\mathrm{i}\left(\left(\widetilde{\nu}\left(\varepsilon_{1}, \varepsilon_{2}\right)+1\right)-\frac{1}{2}\right) \ln 12+(-1)^{\varepsilon_{2}} a \ln (2+\sqrt{3}) \\
& +\frac{\pi}{4}-\frac{3 \pi}{2}\left(\widetilde{\nu}\left(\varepsilon_{1}, \varepsilon_{2}\right)+1\right)+\mathrm{i} \ln \left(\frac{g_{11}\left(\varepsilon_{1}, \varepsilon_{2}\right) g_{12}\left(\varepsilon_{1}, \varepsilon_{2}\right) \Gamma\left(\widetilde{\nu}\left(\varepsilon_{1}, \varepsilon_{2}\right)+1\right)}{\sqrt{2 \pi}}\right)+\mathcal{O}\left(\tau^{-\delta_{G}} \ln \tau\right), \quad(2.5)
\end{aligned}
$$

with

$$
\begin{gathered}
\phi(\tau)=3 \sqrt{3}(-1)^{\varepsilon_{2}}(\epsilon b)^{1 / 3} \tau^{2 / 3}, \\
\vartheta_{0}=-\frac{\pi}{2}+\frac{\mathrm{i}}{2} \ln (2+\sqrt{3}),
\end{gathered}
$$

and $\Gamma(\cdot)$ is the Euler gamma function 11 .

Let $\mathcal{H}(\tau)$ be the Hamiltonian function defined by Equation (1.2) corresponding to the function $u(\tau)$ given above. Then $\mathcal{H}(\tau)$ has the asymptotic expansion

$$
\begin{gathered}
\mathcal{H}(\tau) \underset{\tau \rightarrow \infty \mathrm{e}^{\mathrm{i} \pi \varepsilon_{1}}}{=} 3(\epsilon b)^{2 / 3} \tau^{1 / 3}-\mathrm{i}(-1)^{\varepsilon_{2}} 4 \sqrt{3}(\epsilon b)^{1 / 3} \tau^{-1 / 3}\left(\left(\widetilde{\nu}\left(\varepsilon_{1}, \varepsilon_{2}\right)+1\right)-\frac{1}{2}+\frac{1}{2 \sqrt{3}}\left(\mathrm{i}(-1)^{\varepsilon_{2}} a+\frac{1}{2}\right)\right. \\
\left.+\frac{\mathrm{i}}{4} \cot \left(\frac{1}{2} \vartheta\left(\varepsilon_{1}, \varepsilon_{2}, \tau\right)\right)+\frac{\mathrm{i}}{4} \cot \left(\frac{1}{2} \vartheta\left(\varepsilon_{1}, \varepsilon_{2}, \tau\right)-\vartheta_{0}\right)+\mathcal{O}\left(\tau^{-\delta_{G}}\right)\right) .
\end{gathered}
$$

The function $f(\tau)$ defined by Equation (1.3) has the following asymptotics:

$$
f(\tau) \underset{\tau \rightarrow \infty \mathrm{e}^{\mathrm{i} \pi \varepsilon_{1}}}{=}-\frac{(-1)^{\varepsilon_{2}}(\epsilon b)^{1 / 3}}{2} \tau^{2 / 3}\left(\mathrm{i}+\frac{3}{\sqrt{2} \sin \left(\frac{1}{2} \vartheta\left(\varepsilon_{1}, \varepsilon_{2}, \tau\right)\right) \sin \left(\frac{1}{2} \vartheta\left(\varepsilon_{1}, \varepsilon_{2}, \tau\right)-\vartheta_{0}\right)}\right) .
$$

Remark 2.2. Define the strip (in the $\phi$-plane)

$$
\mathcal{D}:=\left\{\tau \in \mathbb{C}: \operatorname{Re}(\phi(\tau))>c_{1},|\operatorname{Im}(\phi(\tau))|<c_{2}\right\},
$$

where $\phi(\tau)$ is given in Equation (2.6), and $c_{1}, c_{2}>0$ are parameters. In terms of the original variable $\tau$, the strip $\mathcal{D}$ is a simply-connected domain with convex boundary of increasing width proportional to $|\tau|^{1 / 3}$. The asymptotics of $u(\tau), \mathcal{H}(\tau)$, and $f(\tau)$ presented in Theorem 2.1 are actually valid in the strip domain $\mathcal{D}$. 
Theorem 2.2. Let $\varepsilon_{1}, \varepsilon_{2}=0, \pm 1, \epsilon b=|\epsilon b| \mathrm{e}^{\mathrm{i} \pi \varepsilon_{2}}$, and $u(\tau)$ be a solution of Equation (1.1) corresponding to the monodromy data $\left(a, s_{0}^{0}, s_{0}^{\infty}, s_{1}^{\infty}, g_{11}, g_{12}, g_{21}, g_{22}\right)$. Suppose that

$$
g_{11}\left(\varepsilon_{1}, \varepsilon_{2}\right) g_{12}\left(\varepsilon_{1}, \varepsilon_{2}\right) g_{21}\left(\varepsilon_{1}, \varepsilon_{2}\right) g_{22}\left(\varepsilon_{1}, \varepsilon_{2}\right) \neq 0, \quad \operatorname{Re}\left(\frac{\mathrm{i}}{2 \pi} \ln \left(g_{11}\left(\varepsilon_{1}, \varepsilon_{2}\right) g_{22}\left(\varepsilon_{1}, \varepsilon_{2}\right)\right)\right)=\frac{1}{2} .
$$

Let the branch of the function $\ln (\cdot)$ be chosen 5 such that $\operatorname{Im}\left(\ln \left(-g_{11}\left(\varepsilon_{1}, \varepsilon_{2}\right) g_{22}\left(\varepsilon_{1}, \varepsilon_{2}\right)\right)\right)=0$. Define

$$
\varrho_{1}\left(\varepsilon_{1}, \varepsilon_{2}\right):=\frac{1}{2 \pi} \ln \left(-g_{11}\left(\varepsilon_{1}, \varepsilon_{2}\right) g_{22}\left(\varepsilon_{1}, \varepsilon_{2}\right)\right) \quad(\in \mathbb{R}) .
$$

Then $\exists \delta \in(0,1 / 39)$ such that the function $u(\tau)$ has, for all large enough $m \in \mathbb{N}$, second-order poles, $\tau_{m}^{\infty}$, accumulating at the point at infinity,

$$
\tau_{m}^{\infty} \underset{m \rightarrow \infty}{=} \mathrm{e}^{\mathrm{i} \pi \varepsilon_{1}}\left(\frac{2 \pi(-1)^{\varepsilon_{2}} m}{3 \sqrt{3}(\epsilon b)^{1 / 3}}\right)^{3 / 2}\left(1-\frac{3 \varrho_{1}\left(\varepsilon_{1}, \varepsilon_{2}\right)}{4 \pi} \frac{\ln m}{m}-\frac{3 \varrho_{2}\left(\varepsilon_{1}, \varepsilon_{2}\right)}{4 \pi} \frac{1}{m}\right)+\mathcal{O}\left(m^{\frac{1}{2}-\frac{3 \delta}{2}}\right)
$$

where

$$
\begin{aligned}
\varrho_{2}\left(\varepsilon_{1}, \varepsilon_{2}\right): & =\varrho_{1}\left(\varepsilon_{1}, \varepsilon_{2}\right) \ln (24 \pi)+(-1)^{\varepsilon_{2}} \operatorname{Re}(a) \ln (2+\sqrt{3})+\frac{\pi}{2}-\frac{1}{2} \arg \left(\frac{g_{11}\left(\varepsilon_{1}, \varepsilon_{2}\right) g_{12}\left(\varepsilon_{1}, \varepsilon_{2}\right)}{g_{21}\left(\varepsilon_{1}, \varepsilon_{2}\right) g_{22}\left(\varepsilon_{1}, \varepsilon_{2}\right)}\right) \\
& -\arg \left(\Gamma\left(\frac{1}{2}+\mathrm{i} \varrho_{1}\left(\varepsilon_{1}, \varepsilon_{2}\right)\right)\right)+\mathrm{i}\left((-1)^{\varepsilon_{2}} \operatorname{Im}(a) \ln (2+\sqrt{3})+\frac{1}{2} \ln \left(\left|\frac{g_{11}\left(\varepsilon_{1}, \varepsilon_{2}\right) g_{12}\left(\varepsilon_{1}, \varepsilon_{2}\right)}{g_{21}\left(\varepsilon_{1}, \varepsilon_{2}\right) g_{22}\left(\varepsilon_{1}, \varepsilon_{2}\right)}\right|\right)\right)
\end{aligned}
$$

furthermore, the function $u(\tau)$ has, for all large enough $m \in \mathbb{N}$, a pair of first-order zeroes, $\tau_{m}^{ \pm}$, accumulating at the point at infinity,

$$
\tau_{m}^{ \pm} \underset{m \rightarrow \infty}{=} \mathrm{e}^{\mathrm{i} \pi \varepsilon_{1}}\left(\frac{2 \pi(-1)^{\varepsilon_{2}} m}{3 \sqrt{3}(\epsilon b)^{1 / 3}}\right)^{3 / 2}\left(1-\frac{3 \varrho_{1}\left(\varepsilon_{1}, \varepsilon_{2}\right)}{4 \pi} \frac{\ln m}{m}-\frac{3}{4 \pi}\left(\varrho_{2}\left(\varepsilon_{1}, \varepsilon_{2}\right) \pm 2 \vartheta_{0}\right) \frac{1}{m}\right)+\mathcal{O}\left(m^{\frac{1}{2}-\frac{3 \delta}{2}}\right)
$$

where $\vartheta_{0}$ is given in Equation (2.7).

Remark 2.3. Further information concerning the Laurent expansions of the functions $u(\tau), \mathcal{H}(\tau)$, and $f(\tau)$ at poles and zeroes can be found in Appendix A.

Remark 2.4. To present asymptotics of $u(\tau), \mathcal{H}(\tau)$, and $f(\tau)$ outside of neighborhoods of poles and zeroes, introduce the technical notion of the cheese-like domain, $\mathcal{D}_{u}$, for a solution $u(\tau)$ :

$$
\mathcal{D}_{u}:=\left\{\tau \in \mathcal{D}:\left|\phi(\tau)-\phi\left(\tau_{m}^{\kappa}\right)\right| \geqslant C\left|\tau_{m}^{\kappa}\right|^{-\delta}\right\}
$$

where the strip domain $\mathcal{D}$ is defined by Equation (2.10), $\phi(\tau)$ is given in Equation (2.6), $C>0$ is a parameter, $\kappa=\infty, \pm\left(\tau_{m}^{\kappa}\right.$ are the poles and zeroes introduced in Theorem 2.2), and $0<\delta<1 / 39$. In terms of the variable $\phi$, the cheese-like domain $\mathcal{D}_{u}$ is a multiply-connected domain which resembles $\mathcal{D}$ with circular "cheese-holes" centered at $\phi\left(\tau_{m}^{\kappa}\right)$ of shrinking radii, whilst in terms of the original variable $\tau$ the diameter of the cheese-holes are increasing, that is, $\left|\tau-\tau_{m}^{\kappa}\right| \sim\left|\tau_{m}^{\kappa}\right|^{\frac{1}{3}-\delta}$.

Theorem 2.3. Let $\varepsilon_{1}, \varepsilon_{2}=0, \pm 1, \epsilon b=|\epsilon b| \mathrm{e}^{\mathrm{i} \pi \varepsilon_{2}}$, and $u(\tau)$ be a solution of Equation (1.1) corresponding to the monodromy data $\left(a, s_{0}^{0}, s_{0}^{\infty}, s_{1}^{\infty}, g_{11}, g_{12}, g_{21}, g_{22}\right)$. Suppose that conditions (2.11) are valid, the branch of $\ln (\cdot)$ is chosen as in Theorem 2.2, and $\varrho_{1}\left(\varepsilon_{1}, \varepsilon_{2}\right)$ is defined by Equation (2.12). Then there exist $\delta, \delta_{G} \in \mathbb{R}_{+}$satisfying the inequalities

$$
0<\delta<\frac{1}{39}, \quad 0<\delta<\delta_{G}<\frac{1}{15}-\frac{8 \delta}{5}
$$

such that $u(\tau)$ has the asymptotic expansion

$$
u(\tau) \underset{\substack{\tau \rightarrow \infty e^{\mathrm{i} \pi \varepsilon_{1}} \\ \tau \in \mathcal{D}_{u}}}{=} \frac{\epsilon(\epsilon b)^{2 / 3}}{2} \tau^{1 / 3} \frac{\sin \left(\frac{1}{2} \theta\left(\varepsilon_{1}, \varepsilon_{2}, \tau\right)-\vartheta_{0}\right) \sin \left(\frac{1}{2} \theta\left(\varepsilon_{1}, \varepsilon_{2}, \tau\right)+\vartheta_{0}\right)}{\sin ^{2}\left(\frac{1}{2} \theta\left(\varepsilon_{1}, \varepsilon_{2}, \tau\right)\right)},
$$

\footnotetext{
${ }^{5}$ The second condition of Equations 2.11 suggests that this branch of $\ln (\cdot)$ exists.
} 
where

$$
\begin{aligned}
\theta\left(\varepsilon_{1}, \varepsilon_{2}, \tau\right): & =\phi(\tau)+\varrho_{1}\left(\varepsilon_{1}, \varepsilon_{2}\right) \ln \phi(\tau)+\varrho_{1}\left(\varepsilon_{1}, \varepsilon_{2}\right) \ln 12+(-1)^{\varepsilon_{2}} \operatorname{Re}(a) \ln (2+\sqrt{3})+\frac{\pi}{2} \\
& -\frac{1}{2} \arg \left(\frac{g_{11}\left(\varepsilon_{1}, \varepsilon_{2}\right) g_{12}\left(\varepsilon_{1}, \varepsilon_{2}\right)}{g_{21}\left(\varepsilon_{1}, \varepsilon_{2}\right) g_{22}\left(\varepsilon_{1}, \varepsilon_{2}\right)}\right)-\arg \left(\Gamma\left(\frac{1}{2}+\mathrm{i} \varrho_{1}\left(\varepsilon_{1}, \varepsilon_{2}\right)\right)\right)+\mathrm{i}\left((-1)^{\varepsilon_{2}} \operatorname{Im}(a) \ln (2+\sqrt{3})\right. \\
& \left.+\frac{1}{2} \ln \left(\left|\frac{g_{11}\left(\varepsilon_{1}, \varepsilon_{2}\right) g_{12}\left(\varepsilon_{1}, \varepsilon_{2}\right)}{g_{21}\left(\varepsilon_{1}, \varepsilon_{2}\right) g_{22}\left(\varepsilon_{1}, \varepsilon_{2}\right)}\right|\right)\right)+\mathcal{O}\left(\tau^{-\delta_{G}} \ln \tau\right),
\end{aligned}
$$

with $\phi(\tau)$ and $\vartheta_{0}$ given, respectively, in Equations (2.6) and (2.7).

Let $\mathcal{H}(\tau)$ be the Hamiltonian function defined by Equation (1.2) corresponding to the function $u(\tau)$ given above. Then $\mathcal{H}(\tau)$ has the asymptotic expansion

$$
\begin{gathered}
\mathcal{H}(\tau) \underset{\substack{\tau \rightarrow \mathfrak{c}_{\tau \in \mathcal{D}_{u}}^{\mathrm{i} \pi \varepsilon_{1}} \\
\tau \in}}{=} 3(\epsilon b)^{2 / 3} \tau^{1 / 3}+(-1)^{\varepsilon_{2}} 4 \sqrt{3}(\epsilon b)^{1 / 3} \tau^{-1 / 3}\left(\varrho_{1}\left(\varepsilon_{1}, \varepsilon_{2}\right)+\frac{1}{2 \sqrt{3}}\left((-1)^{\varepsilon_{2}} a-\frac{\mathrm{i}}{2}\right)\right. \\
\left.+\frac{1}{4} \cot \left(\frac{1}{2} \theta\left(\varepsilon_{1}, \varepsilon_{2}, \tau\right)\right)+\frac{1}{4} \cot \left(\frac{1}{2} \theta\left(\varepsilon_{1}, \varepsilon_{2}, \tau\right)-\vartheta_{0}\right)+\mathcal{O}\left(\tau^{-\delta_{G}}\right)\right) .
\end{gathered}
$$

The function $f(\tau)$ defined by Equation (1.3) has the following asymptotics:

$$
f(\tau) \underset{\substack{\tau \rightarrow \infty \mathcal{i}^{i \pi \varepsilon_{1}} \\ \tau \in \mathcal{D}_{u}}}{=}-\frac{(-1)^{\varepsilon_{2}}(\epsilon b)^{1 / 3}}{2} \tau^{2 / 3}\left(\mathrm{i}+\frac{3}{\sqrt{2} \sin \left(\frac{1}{2} \theta\left(\varepsilon_{1}, \varepsilon_{2}, \tau\right)\right) \sin \left(\frac{1}{2} \theta\left(\varepsilon_{1}, \varepsilon_{2}, \tau\right)-\vartheta_{0}\right)}\right) .
$$

Remark 2.5. For real, non-zero values of $b$, singular real solutions $u(\tau)$ of Equation (1.1) are specified by the following "singular real reduction" for the monodromy data:

$$
\begin{gathered}
s_{0}^{0}=-\overline{s_{0}^{0}}, \quad s_{0}^{\infty}\left(\varepsilon_{1}, \varepsilon_{2}\right)=-\overline{s_{1}^{\infty}\left(\varepsilon_{1}, \varepsilon_{2}\right)} \mathrm{e}^{2 \pi a}, \quad g_{11}\left(\varepsilon_{1}, \varepsilon_{2}\right)=-\overline{g_{22}\left(\varepsilon_{1}, \varepsilon_{2}\right)}, \\
g_{12}\left(\varepsilon_{1}, \varepsilon_{2}\right)=-\overline{g_{21}\left(\varepsilon_{1}, \varepsilon_{2}\right)}, \quad \operatorname{Im}(a)=0 .
\end{gathered}
$$

In this case, asymptotics of $\tau_{m}^{\infty}, \tau_{m}^{ \pm}, u(\tau), \mathcal{H}(\tau)$, and $f(\tau)$ are as given in Equations (2.13), (2.15), (2.16), (2.18), and (2.19), respectively, but with the changes $\varrho_{1}\left(\varepsilon_{1}, \varepsilon_{2}\right) \rightarrow \varrho_{0}\left(\varepsilon_{1}, \varepsilon_{2}\right), \varrho_{2}\left(\varepsilon_{1}, \varepsilon_{2}\right) \rightarrow$ $\varrho_{0}^{\sharp}\left(\varepsilon_{1}, \varepsilon_{2}\right)$, and $\theta\left(\varepsilon_{1}, \varepsilon_{2}, \tau\right) \rightarrow \Theta_{0}\left(\varepsilon_{1}, \varepsilon_{2}, \tau\right)$, where

$$
\begin{aligned}
\varrho_{0}\left(\varepsilon_{1}, \varepsilon_{2}\right) & :=\frac{1}{\pi} \ln \left|g_{11}\left(\varepsilon_{1}, \varepsilon_{2}\right)\right|, \\
\varrho_{0}^{\sharp}\left(\varepsilon_{1}, \varepsilon_{2}\right) & :=\varrho_{0}\left(\varepsilon_{1}, \varepsilon_{2}\right) \ln (24 \pi)+(-1)^{\varepsilon_{2}} \operatorname{Re}(a) \ln (2+\sqrt{3})-\frac{\pi}{2} \\
& -\arg \left(g_{11}\left(\varepsilon_{1}, \varepsilon_{2}\right) g_{12}\left(\varepsilon_{1}, \varepsilon_{2}\right) \Gamma\left(\frac{1}{2}+\mathrm{i} \varrho_{0}\left(\varepsilon_{1}, \varepsilon_{2}\right)\right)\right), \\
\Theta_{0}\left(\varepsilon_{1}, \varepsilon_{2}, \tau\right) & :=\phi(\tau)+\varrho_{0}\left(\varepsilon_{1}, \varepsilon_{2}\right) \ln \phi(\tau)+\varrho_{0}\left(\varepsilon_{1}, \varepsilon_{2}\right) \ln 12+(-1)^{\varepsilon_{2}} \operatorname{Re}(a) \ln (2+\sqrt{3}) \\
& -\frac{\pi}{2}-\arg \left(g_{11}\left(\varepsilon_{1}, \varepsilon_{2}\right) g_{12}\left(\varepsilon_{1}, \varepsilon_{2}\right) \Gamma\left(\frac{1}{2}+\mathrm{i} \varrho_{0}\left(\varepsilon_{1}, \varepsilon_{2}\right)\right)\right)+\mathcal{O}\left(\tau^{-\delta_{G}} \ln \tau\right) .
\end{aligned}
$$

\section{Asymptotic Solution of the Direct Monodromy Problem}

\subsection{Notation}

In this section the monodromy data introduced in Section 1 is calculated as $\tau \rightarrow+\infty$ for $\epsilon b>0$ (corresponding to $\varepsilon_{1}=\varepsilon_{2}=0$ ): this constitutes the first step towards the proof of the results stated in Section 2. This calculation consists of three components: (i) the WKB analysis of the $\mu$-part of System (1.4), that is,

$$
\partial_{\mu} \Psi(\mu)=\widetilde{U}(\mu, \tau) \Psi(\mu),
$$

where $\Psi(\mu)=\Psi(\mu, \tau)$; (ii) the approximation of $\Psi(\mu)$ in the neighborhoods of the turning points; and (iii) the matching of these asymptotics.

\footnotetext{
${ }^{6}$ There exist regular real solutions (cf. Part I [1]) which are specified by a different real reduction: we plan to discuss this in a subsequent work.
} 
Some solutions $u(\tau)$ of Equation (1.1) might (and actually do) have poles and zeroes located on the positive real semi-axis. In order to be able to study such solutions, one must consider a slightly more general complex domain $\widetilde{\mathcal{D}}_{u}$ : this coincides with the cheese-like domain $\mathcal{D}_{u}$ introduced in Remark 2.4 however, since, a priori, one does not know the solutions $u(\tau)$ that have such poles and zeroes, nor the exact locations of these poles and zeroes (cf. Equations (2.13) and (2.15)), it is necessary to introduce a formal definition for $\widetilde{\mathcal{D}}_{u}$. Denote by $\mathcal{P}_{u}$ and $\mathcal{Z}_{u}$, respectively, the countable sets of poles and zeroes of $u(\tau)$ : as follows from the Painlevé property, these sets might have accumulation points at 0 and $\infty$. Define neighborhoods of $\mathcal{P}_{u}$ and $\mathcal{Z}_{u}$, respectively: for $\delta>0$,

$$
\begin{aligned}
& \mathcal{P}_{u}^{\delta}:=\left\{\tau \in \mathbb{C}:\left|\phi(\tau)-\phi\left(\tau_{p}\right)\right|>C\left|\tau_{p}\right|^{-\delta}, \tau_{p} \in \mathcal{P}_{u}\right\}, \\
& \mathcal{Z}_{u}^{\delta}:=\left\{\tau \in \mathbb{C}:\left|\phi(\tau)-\phi\left(\tau_{z}\right)\right|>C\left|\tau_{z}\right|^{-\delta}, \tau_{z} \in \mathcal{Z}_{u}\right\} .
\end{aligned}
$$

Now, the cheese-like domain $\widetilde{\mathcal{D}}_{u}$ is defined:

$$
\widetilde{\mathcal{D}}_{u}:=\mathcal{D} \backslash\left(\mathcal{P}_{u}^{\delta} \cup \mathcal{Z}_{u}^{\delta}\right),
$$

where $\mathcal{D}$ is given in Equation (2.10).

Remark 3.1.1. Throughout this section, and for brevity of notation, the following convention is adopted: in asymptotics of all expressions and formulae depending on $u(\tau)$, the "notation" $\tau \rightarrow+\infty$ means $\operatorname{Re}(\tau) \rightarrow+\infty$ and $\tau \in \widetilde{\mathcal{D}}_{u}$.

Further notation used throughout this section is now summarized:

(1) $\mathrm{I}=\left(\begin{array}{ll}1 & 0 \\ 0 & 1\end{array}\right)$ is the $2 \times 2$ identity matrix, $\sigma_{1}=\left(\begin{array}{ll}0 & 1 \\ 1 & 0\end{array}\right), \sigma_{2}=\left(\begin{array}{cc}0 & -\mathrm{i} \\ \mathrm{i} & 0\end{array}\right)$, and $\sigma_{3}=\left(\begin{array}{cc}1 & 0 \\ 0 & -1\end{array}\right)$ are the Pauli matrices, $\sigma_{ \pm}:=\frac{1}{2}\left(\sigma_{1} \pm \mathrm{i} \sigma_{2}\right)$ are the raising $(+)$ and lowering $(-)$ matrices, and $\mathbb{R}_{ \pm}:=\{x \in \mathbb{R}: \pm x>$ $0\}$

(2) for $\left(\varsigma_{1}, \varsigma_{2}\right) \in \mathbb{R} \times \mathbb{R}$, the function $\left(z-\varsigma_{1}\right)^{\mathrm{i} \varsigma_{2}}: \mathbb{C} \backslash\left(-\infty, \varsigma_{1}\right) \rightarrow \mathbb{C}, z \mapsto \exp \left(\mathrm{i} \varsigma_{2} \ln \left(z-\varsigma_{1}\right)\right)$, with the branch cut taken along $\left(-\infty, \varsigma_{1}\right)$ and the principal branch of the logarithm chosen;

(3) for a scalar $\omega_{o}$ and a $2 \times 2$ matrix $\hat{\Upsilon}, \omega_{o}^{\operatorname{ad}\left(\sigma_{3}\right)} \hat{\Upsilon}:=\omega_{o}^{\sigma_{3}} \hat{\Upsilon} \omega_{o}^{-\sigma_{3}}$;

(4) for a $2 \times 2$ matrix-valued function $\mathfrak{Y}(z), \mathfrak{Y}(z)=_{z \rightarrow z_{0}} \mathcal{O}(*)$ (resp., $o(*)$ ) means $\mathfrak{Y}_{i j}(z)=_{z \rightarrow z_{0}} \mathcal{O}\left(*_{i j}\right)$ (resp., $\left.o\left(*_{i j}\right)\right), i, j=1,2$;

(5) for $\mathfrak{B}(\cdot) \in \mathrm{M}_{2}(\mathbb{C}),\|\mathfrak{B}(\cdot)\|:=\left(\sum_{i, j=1}^{2} \mathfrak{B}_{i j}(\cdot) \overline{\mathfrak{B}_{i j}(\cdot)}\right)^{1 / 2}$ denotes the Hilbert-Schmidt norm, where $\mp$ denotes complex conjugation of $\star ;$

(6) for some $\delta_{*}>0$ and sufficiently small, $\mathcal{O}_{\delta_{*}}(p)$ denotes the $\delta_{*}$-neighborhood of the point $p$, that is, $\mathcal{O}_{\delta_{*}}(p):=\left\{z \in \mathbb{C}:|z-p|<\delta_{*}\right\}$.

\subsection{WKB Analysis}

This subsection is devoted to the WKB analysis of Equation (3.1) as $\tau \rightarrow+\infty$ (and $\epsilon b>0$ ). In order to transform Equation (3.1) into a form amenable to WKB analysis, one uses the result of Proposition 4.1.1 in [1, which is summarized here for the reader's convenience.

Proposition 3.2.1 ([1]). Let

$$
\begin{gathered}
A(\tau)=a(\tau) \tau^{-2 / 3}, \quad B(\tau)=b(\tau) \tau^{-2 / 3}, \quad C(\tau)=c(\tau) \tau^{-1 / 3}, \quad D(\tau)=d(\tau) \tau^{-1 / 3}, \\
\widetilde{\mu}=\mu \tau^{1 / 6}, \quad \widetilde{\Psi}(\widetilde{\mu}):=\tau^{-(1 / 12) \sigma_{3}} \Psi\left(\widetilde{\mu} \tau^{-1 / 6}\right),
\end{gathered}
$$

where $\widetilde{\Psi}(\widetilde{\mu})=\widetilde{\Psi}(\widetilde{\mu}, \tau)$. Then

$$
\partial_{\widetilde{\mu}} \widetilde{\Psi}(\widetilde{\mu})=\tau^{2 / 3} \mathcal{A}(\widetilde{\mu}, \tau) \widetilde{\Psi}(\widetilde{\mu}),
$$

where

$$
\mathcal{A}(\widetilde{\mu}, \tau):=-2 \mathrm{i} \widetilde{\mu} \sigma_{3}+\left(\begin{array}{cc}
0 & -\frac{4 \mathrm{i} \sqrt{-a(\tau) b(\tau)}}{b(\tau)} \\
-2 d(\tau) & 0
\end{array}\right)-\frac{\mathrm{i} r(\tau)(\epsilon b)^{1 / 3}}{2 \widetilde{\mu}} \sigma_{3}+\frac{1}{\widetilde{\mu}^{2}}\left(\begin{array}{cc}
0 & \frac{\mathrm{i} \epsilon b}{b(\tau)} \\
\mathrm{i} b(\tau) & 0
\end{array}\right),
$$

with

$$
\frac{\mathrm{i} r(\tau)(\epsilon b)^{1 / 3}}{2}=\left(\mathrm{i} a+\frac{1}{2}\right) \tau^{-2 / 3}+\frac{2 a(\tau) d(\tau)}{\sqrt{-a(\tau) b(\tau)}} .
$$


It is now important to state under what conditions the WKB analysis of Equation (3.6) is undertaken. Define the functions $\hat{r}_{0}(\tau), \hat{u}_{0}(\tau)$, and $h_{0}(\tau)$ via the following equations:

$$
\begin{aligned}
\sqrt{-a(\tau) b(\tau)}+c(\tau) d(\tau)+\frac{a(\tau) d(\tau) \tau^{-2 / 3}}{2 \sqrt{-a(\tau) b(\tau)}} & -\frac{1}{4}\left(a-\frac{\mathrm{i}}{2}\right)^{2} \tau^{-4 / 3}=\frac{3}{4}(\epsilon b)^{2 / 3}-h_{0}(\tau) \tau^{-2 / 3}, \\
r(\tau) & =-2+\hat{r}_{0}(\tau), \\
\sqrt{-a(\tau) b(\tau)} & =\frac{(\epsilon b)^{2 / 3}}{2}\left(1+\hat{u}_{0}(\tau)\right),
\end{aligned}
$$

and assume that the functions $\hat{r}_{0}(\tau), \hat{u}_{0}(\tau)$, and $h_{0}(\tau)$, which are holomorphic in the domain $\widetilde{\mathcal{D}}_{u}$, satisfy the conditions

$$
\begin{gathered}
\mathcal{O}\left(\tau^{-\frac{1}{3}+\delta_{1}}\right) \underset{\tau \rightarrow+\infty}{\leqslant}\left|\hat{r}_{0}(\tau)\right| \underset{\tau \rightarrow+\infty}{\leqslant} \mathcal{O}\left(\tau^{\delta}\right), \quad \mathcal{O}\left(\tau^{-\frac{1}{3}+\delta_{1}}\right) \underset{\tau \rightarrow+\infty}{\leqslant}\left|\hat{u}_{0}(\tau)\right| \underset{\tau \rightarrow+\infty}{\leqslant} \mathcal{O}\left(\tau^{2 \delta}\right), \\
\frac{1}{\left|1+\hat{u}_{0}(\tau)\right|} \underset{\tau \rightarrow+\infty}{\leqslant} \mathcal{O}\left(\tau^{\delta}\right), \quad\left|h_{0}(\tau)\right| \underset{\tau \rightarrow+\infty}{\leqslant} \mathcal{O}\left(\tau^{\delta}\right), \quad 0<\delta_{1}<\frac{1}{3}, \quad 0 \leqslant \delta<\frac{1}{39},
\end{gathered}
$$

where the parameter $\delta$ is the one that was introduced in the definition of the cheese-like domain $\widetilde{\mathcal{D}}_{u}$ (cf. Equations (3.2), (3.3), and (3.4)).

Remark 3.2.1. Even though, at this juncture, the upper bound 1/39 for the growth exponent, $\delta$, given in conditions (3.12) might seem artificial, it must be noted that it arises naturally during the course of the ensuing asymptotic analysis.

It is also assumed that the functions $a(\tau), b(\tau), c(\tau)$, and $d(\tau)$ are related via the "integral of motion" associated with the underlying Hamiltonian structure of Equation (1.1) (see [1], Lemma 2.1):

$$
a(\tau) d(\tau)+b(\tau) c(\tau)+\mathrm{i} a \sqrt{-a(\tau) b(\tau)} \tau^{-2 / 3}=-\frac{\mathrm{i} \epsilon b}{2}, \quad \epsilon= \pm 1
$$

Remark 3.2.2. It is worth noting that Equations (3.9)-3.11) and (3.13) are self-consistent; in fact, they are equivalent to

$$
\begin{aligned}
a(\tau) d(\tau) & =\frac{(\epsilon b)^{2 / 3}}{2}\left(1+\hat{u}_{0}(\tau)\right)\left(-\frac{\mathrm{i}(\epsilon b)^{1 / 3}}{2}+\frac{\mathrm{i}(\epsilon b)^{1 / 3} \hat{r}_{0}(\tau)}{4}-\frac{\mathrm{i}}{2}\left(a-\frac{\mathrm{i}}{2}\right) \tau^{-2 / 3}\right) \\
b(\tau) c(\tau) & =\frac{(\epsilon b)^{2 / 3}}{2}\left(1+\hat{u}_{0}(\tau)\right)\left(-\frac{\mathrm{i}(\epsilon b)^{1 / 3}}{2}+\mathrm{i}(\epsilon b)^{1 / 3}\left(\frac{\hat{u}_{0}(\tau)}{1+\hat{u}_{0}(\tau)}-\frac{\hat{r}_{0}(\tau)}{4}\right)-\frac{\mathrm{i}}{2}\left(a+\frac{\mathrm{i}}{2}\right) \tau^{-2 / 3}\right) \\
-h_{0}(\tau) \tau^{-2 / 3} & =\frac{(\epsilon b)^{2 / 3}}{2}\left(\frac{\left(\hat{u}_{0}(\tau)\right)^{2}+\frac{1}{2} \hat{u}_{0}(\tau) \hat{r}_{0}(\tau)}{1+\hat{u}_{0}(\tau)}-\frac{\left(\hat{r}_{0}(\tau)\right)^{2}}{8}\right)+\frac{(\epsilon b)^{1 / 3}}{2}\left(a-\frac{\mathrm{i}}{2}\right) \frac{\tau^{-2 / 3}}{\left(1+\hat{u}_{0}(\tau)\right)}
\end{aligned}
$$

furthermore, via Equations (3.11), (3.14), and (3.15),

$$
\begin{aligned}
c(\tau) d(\tau) & =-\left(\frac{\mathrm{i}(\epsilon b)^{1 / 3}}{2}-\frac{\mathrm{i}(\epsilon b)^{1 / 3} \hat{r}_{0}(\tau)}{4}+\frac{\mathrm{i}}{2}\left(a-\frac{\mathrm{i}}{2}\right) \tau^{-2 / 3}\right) \\
& \times\left(\frac{\mathrm{i}(\epsilon b)^{1 / 3}}{2}-\mathrm{i}(\epsilon b)^{1 / 3}\left(\frac{\hat{u}_{0}(\tau)}{1+\hat{u}_{0}(\tau)}-\frac{\hat{r}_{0}(\tau)}{4}\right)+\frac{\mathrm{i}}{2}\left(a+\frac{\mathrm{i}}{2}\right) \tau^{-2 / 3}\right) .
\end{aligned}
$$

In certain domains of the complex $\widetilde{\mu}$-plane (see the discussion below), the leading term of asymptotics (as $\tau \rightarrow+\infty$ ) of a fundamental solution, $\widetilde{\Psi}(\widetilde{\mu})$, of Equation $\underline{3.6}$ is given by the following WKB formula 8 (see, for example, Chapter 5 of [9]),

$$
\widetilde{\Psi}_{\mathrm{WKB}}(\widetilde{\mu})=T(\widetilde{\mu}) \exp \left(-\sigma_{3} \mathrm{i} \tau^{2 / 3} \int^{\widetilde{\mu}} l(\xi) \mathrm{d} \xi-\int^{\widetilde{\mu}} \operatorname{diag}\left((T(\xi))^{-1} \partial_{\xi} T(\xi)\right) \mathrm{d} \xi\right)
$$

\footnotetext{
${ }^{7}$ Equations (63)-(66) in [1] are expressed in terms of the functions $r_{0}(\tau)$ and $u_{0}(\tau)$, whereas Equations (3.9) - (3.11) and conditions (3.12) are expressed in terms of the functions $\hat{r}_{0}(\tau):=r_{0}(\tau) \tau^{-1 / 3}$ and $\hat{u}_{0}(\tau):=u_{0}(\tau) \tau^{-1 / 3}$.

${ }^{8}$ For simplicity of notation, the $\tau$ dependencies of $\widetilde{\Psi}_{\mathrm{WKB}}(\widetilde{\mu}), l(\widetilde{\mu})$, and $T(\widetilde{\mu})$ are suppressed.
} 
where

$$
l(\widetilde{\mu}):=\sqrt{\operatorname{det}(\mathcal{A}(\widetilde{\mu}))}
$$

and $T(\widetilde{\mu})$, which diagonalizes $\mathcal{A}(\widetilde{\mu})$, that is, $(T(\widetilde{\mu}))^{-1} \mathcal{A}(\widetilde{\mu}) T(\widetilde{\mu})=-\mathrm{i} l(\widetilde{\mu}) \sigma_{3}$, is given by

$$
T(\widetilde{\mu})=\frac{\mathrm{i}}{\sqrt{2 \mathrm{i} l(\widetilde{\mu})\left(\mathcal{A}_{11}(\widetilde{\mu})-\mathrm{i} l(\widetilde{\mu})\right)}}\left(\mathcal{A}(\widetilde{\mu})-\mathrm{i} l(\widetilde{\mu}) \sigma_{3}\right) \sigma_{3},
$$

where $\mathcal{A}_{11}(\widetilde{\mu})$ is the (11)-element of the matrix $\mathcal{A}(\widetilde{\mu})$ (cf. Equation (3.7)).

Proposition 3.2.2. Let $T(\widetilde{\mu})$ be given in Equation (3.20), with $\mathcal{A}(\widetilde{\mu})$ and $l(\widetilde{\mu})$ defined by Equations (3.7) and (3.19), respectively. Then $\operatorname{det}(T(\widetilde{\mu}))=1$, and $\operatorname{tr}\left((T(\widetilde{\mu}))^{-1} \partial_{\widetilde{\mu}} T(\widetilde{\mu})\right)=0$; furthermore,

$$
\operatorname{diag}\left((T(\widetilde{\mu}))^{-1} \partial_{\widetilde{\mu}} T(\widetilde{\mu})\right)=-\frac{1}{2}\left(\frac{\mathcal{A}_{12}(\widetilde{\mu}) \partial_{\widetilde{\mu}} \mathcal{A}_{21}(\widetilde{\mu})-\mathcal{A}_{21}(\widetilde{\mu}) \partial_{\widetilde{\mu}} \mathcal{A}_{12}(\widetilde{\mu})}{2 \mathrm{i} l(\widetilde{\mu}) \mathcal{A}_{11}(\widetilde{\mu})+2 l^{2}(\widetilde{\mu})}\right) \sigma_{3}
$$

Proof. Set $T(\widetilde{\mu})=\left(\begin{array}{cc}T_{11}(\widetilde{\mu}) & T_{12}(\widetilde{\mu}) \\ T_{21}(\widetilde{\mu}) & T_{22}(\widetilde{\mu})\end{array}\right)$. From the formula for $T(\widetilde{\mu})$ given in Equation $(\underline{3.20})$, with $\mathcal{A}(\widetilde{\mu})$ and $l(\widetilde{\mu})$ defined by Equations (3.7) and (3.19), respectively, one shows at

$$
\begin{gathered}
T_{11}(\widetilde{\mu})=T_{22}(\widetilde{\mu})=\frac{\mathrm{i}\left(\mathcal{A}_{11}(\widetilde{\mu})-\mathrm{i} l(\widetilde{\mu})\right)}{\sqrt{2 \mathrm{i} l(\mu)\left(\mathcal{A}_{11}(\widetilde{\mu})-\mathrm{i} l(\widetilde{\mu})\right)}}, \quad T_{12}(\widetilde{\mu})=-\frac{\mathrm{i} \mathcal{A}_{12}(\widetilde{\mu})}{\sqrt{2 \mathrm{i} l(\mu)\left(\mathcal{A}_{11}(\widetilde{\mu})-\mathrm{i} l(\widetilde{\mu})\right)}}, \\
T_{21}(\widetilde{\mu})=\frac{\mathrm{i} \mathcal{A}_{21}(\widetilde{\mu})}{\sqrt{2 \mathrm{i} l(\mu)\left(\mathcal{A}_{11}(\widetilde{\mu})-\mathrm{i} l(\widetilde{\mu})\right)}},
\end{gathered}
$$

whence $\left(\operatorname{as} \operatorname{det}(\mathcal{A}(\widetilde{\mu}))=l^{2}(\widetilde{\mu})\right.$ and $\left.\operatorname{tr}(\mathcal{A}(\widetilde{\mu}))=0\right) \operatorname{det}(T(\widetilde{\mu}))=\left(T_{11}(\widetilde{\mu})\right)^{2}-T_{12}(\widetilde{\mu}) T_{21}(\widetilde{\mu})=1$. Since $\operatorname{det}(T(\widetilde{\mu}))=1$, it follows that

$$
(T(\widetilde{\mu}))^{-1} \partial_{\widetilde{\mu}} T(\widetilde{\mu})=\left(\begin{array}{ll}
T_{11}(\widetilde{\mu}) \partial_{\widetilde{\mu}} T_{11}(\widetilde{\mu})-T_{12}(\widetilde{\mu}) \partial_{\widetilde{\mu}} T_{21}(\widetilde{\mu}) & T_{11}(\widetilde{\mu}) \partial_{\widetilde{\mu}} T_{12}(\widetilde{\mu})-T_{12}(\widetilde{\mu}) \partial_{\widetilde{\mu}} T_{11}(\widetilde{\mu}) \\
T_{11}(\widetilde{\mu}) \partial_{\widetilde{\mu}} T_{21}(\widetilde{\mu})-T_{21}(\widetilde{\mu}) \partial_{\widetilde{\mu}} T_{11}(\widetilde{\mu}) & T_{11}(\widetilde{\mu}) \partial_{\widetilde{\mu}} T_{11}(\widetilde{\mu})-T_{21}(\widetilde{\mu}) \partial_{\widetilde{\mu}} T_{12}(\widetilde{\mu})
\end{array}\right),
$$

whence

$$
\operatorname{diag}\left((T(\widetilde{\mu}))^{-1} \partial_{\widetilde{\mu}} T(\widetilde{\mu})\right)=\left(\begin{array}{cc}
\frac{1}{2} \partial_{\widetilde{\mu}}\left(T_{11}(\widetilde{\mu})\right)^{2}-T_{12}(\widetilde{\mu}) \partial_{\widetilde{\mu}} T_{21}(\widetilde{\mu}) & 0 \\
0 & \frac{1}{2} \partial_{\widetilde{\mu}}\left(T_{11}(\widetilde{\mu})\right)^{2}-T_{21}(\widetilde{\mu}) \partial_{\widetilde{\mu}} T_{12}(\widetilde{\mu})
\end{array}\right),
$$

which implies that $\operatorname{tr}\left((T(\widetilde{\mu}))^{-1} \partial_{\widetilde{\mu}} T(\widetilde{\mu})\right)=\partial_{\widetilde{\mu}}\left(\left(T_{11}(\widetilde{\mu})\right)^{2}-T_{12}(\widetilde{\mu}) T_{21}(\widetilde{\mu})\right)=\partial_{\widetilde{\mu}}(1)=0$. Writing out explicitly $\operatorname{diag}\left((T(\widetilde{\mu}))^{-1} \partial_{\widetilde{\mu}} T(\widetilde{\mu})\right)$, one shows that

$$
\begin{aligned}
& \left((T(\widetilde{\mu}))^{-1} \partial_{\widetilde{\mu}} T(\widetilde{\mu})\right)_{11}=\frac{\mathrm{i}}{4}\left(\frac{\partial_{\widetilde{\mu}} \mathcal{A}_{11}(\widetilde{\mu})}{l(\widetilde{\mu})}-\frac{\mathcal{A}_{11}(\widetilde{\mu}) \partial_{\widetilde{\mu}} l(\widetilde{\mu})}{l^{2}(\widetilde{\mu})}\right)-\frac{\mathcal{A}_{12}(\widetilde{\mu}) \partial_{\widetilde{\mu}} \mathcal{A}_{21}(\widetilde{\mu})}{\left(2 \mathrm{i} l(\widetilde{\mu}) \mathcal{A}_{11}(\widetilde{\mu})+2 l^{2}(\widetilde{\mu})\right)} \\
& +\frac{\mathcal{A}_{12}(\widetilde{\mu}) \mathcal{A}_{21}(\widetilde{\mu})\left(\mathrm{i}\left(l(\widetilde{\mu}) \partial_{\widetilde{\mu}} \mathcal{A}_{11}(\widetilde{\mu})+\mathcal{A}_{11}(\widetilde{\mu}) \partial_{\widetilde{\mu}} l(\widetilde{\mu})\right)+2 l(\widetilde{\mu}) \partial_{\widetilde{\mu}} l(\widetilde{\mu})\right)}{\left(2 \mathrm{i} l(\widetilde{\mu}) \mathcal{A}_{11}(\widetilde{\mu})+2 l^{2}(\widetilde{\mu})\right)^{2}}, \\
& \left((T(\widetilde{\mu}))^{-1} \partial_{\widetilde{\mu}} T(\widetilde{\mu})\right)_{22}=\frac{\mathrm{i}}{4}\left(\frac{\partial_{\widetilde{\mu}} \mathcal{A}_{11}(\widetilde{\mu})}{l(\widetilde{\mu})}-\frac{\mathcal{A}_{11}(\widetilde{\mu}) \partial_{\widetilde{\mu}} l(\widetilde{\mu})}{l^{2}(\widetilde{\mu})}\right)-\frac{\mathcal{A}_{21}(\widetilde{\mu}) \partial_{\widetilde{\mu}} \mathcal{A}_{12}(\widetilde{\mu})}{\left(2 \mathrm{i} l(\widetilde{\mu}) \mathcal{A}_{11}(\widetilde{\mu})+2 l^{2}(\widetilde{\mu})\right)} \\
& +\frac{\mathcal{A}_{12}(\widetilde{\mu}) \mathcal{A}_{21}(\widetilde{\mu})\left(\mathrm{i}\left(l(\widetilde{\mu}) \partial_{\widetilde{\mu}} \mathcal{A}_{11}(\widetilde{\mu})+\mathcal{A}_{11}(\widetilde{\mu}) \partial_{\widetilde{\mu}} l(\widetilde{\mu})\right)+2 l(\widetilde{\mu}) \partial_{\widetilde{\mu}} l(\widetilde{\mu})\right)}{\left(2 \mathrm{i} l(\widetilde{\mu}) \mathcal{A}_{11}(\widetilde{\mu})+2 l^{2}(\widetilde{\mu})\right)^{2}}:
\end{aligned}
$$

now, using the fact that $\operatorname{tr}\left((T(\widetilde{\mu}))^{-1} \partial_{\widetilde{\mu}} T(\widetilde{\mu})\right)=0$, one arrives at Equation (3.21).

Corollary 3.2.1. Let $\widetilde{\Psi}_{\mathrm{WKB}}(\widetilde{\mu})$ be given in Equation (3.18), with $l(\widetilde{\mu})$ defined by Equation (3.19) and $T(\widetilde{\mu})$ given in Equation (3.20). Then $\operatorname{det}\left(\widetilde{\Psi}_{\mathrm{WKB}}(\widetilde{\mu})\right)=1$.

The domains in the complex $\widetilde{\mu}$-plane where Equation (3.18) gives the asymptotic approximation of solutions to Equation (3.6) are defined in terms of the Stokes graph (see, for example, [8, 9]). The vertices of the Stokes graph are the singular points of Equation (3.6), that is, $\widetilde{\mu}=0$ and $\widetilde{\mu}=\infty$, and the turning points, which are the roots of the equation $l^{2}(\widetilde{\mu})=0$. The edges of the Stokes graph are the Stokes curves, defined as $\operatorname{Re}\left(\int_{\widetilde{\mu}_{\mathrm{TP}}}^{\widetilde{\mu}} l(\xi) \mathrm{d} \xi\right)=0$, where $\widetilde{\mu}_{\mathrm{TP}}$ denotes a turning point. Canonical domains are those domains in the complex $\widetilde{\mu}$-plane containing one, and only one, Stokes curve and bounded by two adjacent Stokes curves. Note that the restriction of any branch of $l(\widetilde{\mu})$ to a canonical 
domain is a single-valued function. In each canonical domain, for any choice of the branch of $l(\widetilde{\mu})$, there exists a fundamental solution of Equation (3.6) which has asymptotics whose leading term is given by Equation (3.18). From the definition of $l(\widetilde{\mu})$ given by Equation (3.19), one arrives at

$$
l^{2}(\widetilde{\mu})=\frac{4}{\widetilde{\mu}^{4}}\left(\left(\widetilde{\mu}^{2}-\alpha^{2}\right)^{2}\left(\widetilde{\mu}^{2}+2 \alpha^{2}\right)+\left(\widetilde{\mu}^{4}\left(a-\frac{\mathrm{i}}{2}\right)+\widetilde{\mu}^{2} h_{0}(\tau)\right) \tau^{-2 / 3}\right),
$$

where

$$
\alpha:=\frac{(\epsilon b)^{1 / 6}}{\sqrt{2}}>0 .
$$

It follows from Equation (3.23) that there are six turning points: two turning points coalesce (as $\tau \rightarrow+\infty)$ at $\alpha+\mathcal{O}\left(\tau^{-\frac{1}{3}+\frac{\delta}{2}}\right)$, another pair coalesce at $-\alpha+\mathcal{O}\left(\tau^{-\frac{1}{3}+\frac{\delta}{2}}\right)$, and the remaining two turning points approach (as $\tau \rightarrow+\infty) \pm \mathrm{i} \sqrt{2} \alpha+\mathcal{O}\left(\tau^{-\frac{2}{3}+\delta}\right)$, respectively. Denote by $\widetilde{\mu}_{1}$ the turning point in the first quadrant of the complex $\widetilde{\mu}$-plane which approaches $\alpha+\mathcal{O}\left(\tau^{-\frac{1}{3}+\frac{\delta}{2}}\right)$, and by $\widetilde{\mu}_{2}$ the pure imaginary turning point which approaches $\mathrm{i} \sqrt{2} \alpha+\mathcal{O}\left(\tau^{-\frac{2}{3}+\delta}\right)$. Denote by $\mathcal{G}_{1}$ the part of the Stokes graph in the first quadrant of the complex $\widetilde{\mu}$-plane which consists of the vertices $0, \infty, \widetilde{\mu}_{1}$, and $\widetilde{\mu}_{2}$, and the edges $\left(+\mathrm{i} \infty, \widetilde{\mu}_{2}\right),\left(0, \widetilde{\mu}_{1}\right),\left(\widetilde{\mu}_{2}, \widetilde{\mu}_{1}\right)$, and $\left(\widetilde{\mu}_{1},+\infty\right)$ : the complete Stokes graph is the union of the mirror images of $\mathcal{G}_{1}$ with respect to the real and imaginary axes in the complex $\widetilde{\mu}$-plane.

Proposition 3.2.3. Let $l^{2}(\widetilde{\mu})$ be given in Equation (3.23). Then

$$
\int_{\widetilde{\mu}_{0}}^{\widetilde{\mu}} l(\xi) \mathrm{d} \xi \underset{\tau \rightarrow+\infty}{=} \Upsilon_{\tau}(\widetilde{\mu})-\Upsilon_{\tau}\left(\widetilde{\mu}_{0}\right)+\mathcal{O}\left(\varepsilon_{l}(\widetilde{\mu})\right),
$$

where $\widetilde{\mu}_{0} \in \mathbb{C} \backslash\left(\mathcal{O}_{\tau^{-\frac{1}{3}+\frac{\delta}{2}}}( \pm \alpha) \cup \mathcal{O}_{\tau^{-\frac{2}{3}+\delta}}( \pm \mathrm{i} \sqrt{2} \alpha) \cup\{0, \infty\}\right)$ and the path of integration lies in the corresponding 9 canonical domain,

$$
\begin{aligned}
\Upsilon_{\tau}(\xi) & :=\left(\xi+\frac{2 \alpha^{2}}{\xi}\right) \sqrt{\xi^{2}+2 \alpha^{2}}+\tau^{-2 / 3}\left(a-\frac{\mathrm{i}}{2}\right) \ln \left(\xi+\sqrt{\xi^{2}+2 \alpha^{2}}\right) \\
& +\frac{\tau^{-2 / 3}}{2 \sqrt{3}}\left(\left(a-\frac{\mathrm{i}}{2}\right)+\frac{h_{0}(\tau)}{\alpha^{2}}\right) \ln \left(\left(\frac{\sqrt{3} \sqrt{\xi^{2}+2 \alpha^{2}}-\xi+2 \alpha}{\sqrt{3} \sqrt{\xi^{2}+2 \alpha^{2}}+\xi+2 \alpha}\right)\left(\frac{\xi-\alpha}{\xi+\alpha}\right)\right)
\end{aligned}
$$

and $\varepsilon_{l}(\widetilde{\mu})=\varepsilon_{l}^{\natural}(\widetilde{\mu})-\varepsilon_{l}^{\natural}\left(\widetilde{\mu}_{0}\right)$, with

$$
\mathcal{E}_{l}^{\natural}(\xi):= \begin{cases}\frac{\left(c_{1}\left(\delta_{0}\right)\left(h_{0}(\tau)\right)^{2}+c_{2}\left(\delta_{0}\right)\right) \tau^{-4 / 3}}{(\xi \mp \alpha)^{2}}, & \xi \in \mathcal{O}_{\delta_{0}}( \pm \alpha), \\ \frac{\left(c_{3}\left(\delta_{0}\right)\left(h_{0}(\tau)\right)^{2}+c_{4}\left(\delta_{0}\right)\right) \tau^{-4 / 3}}{(\xi \mp \mathrm{i} \sqrt{2} \alpha)^{1 / 2}}, & \xi \in \mathcal{O}_{\delta_{0}}( \pm \mathrm{i} \sqrt{2} \alpha), \\ \left(c_{5}\left(\delta_{0}\right)\left(h_{0}(\tau)\right)^{2}+c_{6}\left(\delta_{0}\right)\right) \tau^{-4 / 3}, & \xi \in \mathcal{O}_{\delta_{0}}(0), \\ \left(c_{7}\left(\delta_{0}\right)\left(h_{0}(\tau)\right)^{2}+c_{8}\left(\delta_{0}\right)\right) \tau^{-4 / 3}, & \xi \in \mathcal{O}_{\delta_{0}}(\infty),\end{cases}
$$

where $c_{k}(z), k=1, \ldots, 8$, are holomorphic functions of $z$ in a neighborhood of $z=0$ with $c_{k}(0) \neq 0$, and $\delta_{0}>0$ and sufficiently small.

Proof. Recall the expression for $l^{2}(\widetilde{\mu})\left(=l^{2}(\widetilde{\mu}, \tau)\right)$ given in Equation (3.23). Set

$$
l_{\infty}^{2}(\widetilde{\mu}):=l^{2}(\widetilde{\mu},+\infty)=4 \widetilde{\mu}^{-4}\left(\widetilde{\mu}^{2}-\alpha^{2}\right)^{2}\left(\widetilde{\mu}^{2}+2 \alpha^{2}\right) .
$$

Define

$$
\Delta_{\tau}(\widetilde{\mu}):=\frac{l^{2}(\widetilde{\mu})-l_{\infty}^{2}(\widetilde{\mu})}{l_{\infty}^{2}(\widetilde{\mu})}=\frac{\widetilde{\mu}^{2}\left(h_{0}(\tau)+\widetilde{\mu}^{2}\left(a-\frac{i}{2}\right)\right) \tau^{-2 / 3}}{\left(\widetilde{\mu}^{2}-\alpha^{2}\right)^{2}\left(\widetilde{\mu}^{2}+2 \alpha^{2}\right)} .
$$

Now, via conditions (3.12), and writing

$$
l(\widetilde{\mu})=l_{\infty}(\widetilde{\mu})\left(1+\Delta_{\tau}(\widetilde{\mu})\right)^{1 / 2} \underset{\tau \rightarrow+\infty}{=} l_{\infty}(\widetilde{\mu})\left(1+\frac{1}{2} \Delta_{\tau}(\widetilde{\mu})+\mathcal{O}\left(\left(\Delta_{\tau}(\widetilde{\mu})\right)^{2}\right)\right)
$$

\footnotetext{
${ }^{9}$ The same canonical domain to which $\widetilde{\mu}_{0}$ belongs.
} 
one arrives at

$$
l(\widetilde{\mu}) \underset{\tau \rightarrow+\infty}{=} 2\left(1-\frac{\alpha^{2}}{\widetilde{\mu}^{2}}\right) \sqrt{\widetilde{\mu}^{2}+2 \alpha^{2}}+\frac{\left(h_{0}(\tau)+\widetilde{\mu}^{2}\left(a-\frac{i}{2}\right)\right) \tau^{-2 / 3}}{\left(\widetilde{\mu}^{2}-\alpha^{2}\right) \sqrt{\widetilde{\mu}^{2}+2 \alpha^{2}}}+\mathcal{O}\left(\frac{\widetilde{\mu}^{2}\left(h_{0}(\tau)+\widetilde{\mu}^{2}\left(a-\frac{i}{2}\right)\right)^{2} \tau^{-4 / 3}}{\left(\widetilde{\mu}^{2}-\alpha^{2}\right)^{3}\left(\widetilde{\mu}^{2}+2 \alpha^{2}\right)^{3 / 2}}\right) .
$$

In order to obtain the leading term in Equation (3.24), one integrates the first two terms in Equation (3.30). Analogously, integrating the error term in Equation (3.30), one finds an explicit expression for the function $\varepsilon_{l}^{\natural}(\widetilde{\mu})$ : since this latter expression is quite cumbersome, only its asymptotics at the singular and turning points are presented in Equation (3.26).

Corollary 3.2.2. Set $\widetilde{\mu}_{0}=\alpha+\tau^{-1 / 3} \widetilde{\Lambda}$, where $\widetilde{\Lambda}=_{\tau \rightarrow+\infty} \mathcal{O}\left(\tau^{\varepsilon}\right), 0<\delta<\varepsilon<1 / 9$. Then

$$
\int_{\widetilde{\mu}_{0}}^{\widetilde{\mu}} l(\xi) \mathrm{d} \xi \underset{\tau \rightarrow+\infty}{=} \Upsilon_{\tau}(\widetilde{\mu})+\Upsilon_{\tau}^{\sharp}+\mathcal{O}\left(\varepsilon_{l}^{\natural}(\widetilde{\mu})\right)+\mathcal{O}\left(\tau^{-\frac{2}{3}-2(\varepsilon-\delta)}\right)+\mathcal{O}\left(\tau^{-1+3 \varepsilon}\right),
$$

where $\Upsilon_{\tau}(\widetilde{\mu})$ and $\mathcal{E}_{l}^{\natural}(\widetilde{\mu})$ are defined in Proposition 3.2 .3

$$
\begin{aligned}
\Upsilon_{\tau}^{\sharp} & :=\mp 3 \sqrt{3} \alpha^{2} \mp 2 \sqrt{3} \tau^{-2 / 3} \widetilde{\Lambda}^{2}-\tau^{-2 / 3}\left(a-\frac{\mathrm{i}}{2}\right) \ln \left((\sqrt{3} \pm 1) \alpha \mathrm{e}^{\mathrm{i} \pi \mathfrak{s}( \pm)}\right) \\
& \mp \frac{\tau^{-2 / 3}}{2 \sqrt{3}}\left(\left(a-\frac{\mathrm{i}}{2}\right)+\frac{h_{0}(\tau)}{\alpha^{2}}\right)\left(\ln \widetilde{\Lambda}-\frac{1}{3} \ln \tau-\ln (3 \alpha)\right),
\end{aligned}
$$

and $\mathfrak{s}( \pm)=(1 \mp 1) / 2$, with the upper (resp., lower) signs taken if the positive $(+)$ (resp., negative $(-))$ branch of the square-root function $\sqrt{\xi^{2}+2 \alpha^{2}}$ is chosen.

Proof. Substituting $\widetilde{\mu}_{0}$ as the argument of the functions $\Upsilon_{\tau}(\xi)$ and $\varepsilon_{l}^{\natural}(\xi)$ (cf. Equation (3.25) and the first line of Equation (3.26), respectively) and expanding with respect to (the small parameter) $\tau^{-1 / 3} \widetilde{\Lambda}$, one arrives at the following estimates:

$$
\Upsilon_{\tau}\left(\widetilde{\mu}_{0}\right) \underset{\tau \rightarrow+\infty}{=} \Upsilon_{\tau}^{\sharp}+\mathcal{O}\left(\tau^{-1} \widetilde{\Lambda}^{3}\right) \quad \text { and } \quad \varepsilon_{l}^{\natural}\left(\widetilde{\mu}_{0}\right) \underset{\tau \rightarrow+\infty}{=} \mathcal{O}\left(\tau^{-2 / 3}\left(c_{1}\left(\delta_{0}\right)\left(h_{0}(\tau)\right)^{2}+c_{2}\left(\delta_{0}\right)\right) \widetilde{\Lambda}^{-2}\right),
$$

where $\Upsilon_{\tau}^{\sharp}$ is defined by Equation (3.32). The inequality $\delta<\varepsilon<1 / 9$ (see conditions (3.12) for the function $\left.h_{0}(\tau)\right)$ is introduced in order to guarantee that the last two error estimates in Equation (3.31) are decaying after multiplication by the large parameter $\tau^{2 / 3}$ (cf. Equation (3.18)).

Proposition 3.2.4. Let $T(\widetilde{\mu})$ be given in Equation (3.20), with $\mathcal{A}(\widetilde{\mu})$ defined by Equation (3.7), and $l^{2}(\widetilde{\mu})$ given in Equation (3.23). Then

$$
\int_{\widetilde{\mu}_{0}}^{\widetilde{\mu}} \operatorname{diag}\left((T(\xi))^{-1} \partial_{\xi} T(\xi)\right) \mathrm{d} \xi \underset{\tau \rightarrow+\infty}{=}\left(\mathcal{I}_{\tau}(\widetilde{\mu})+\mathcal{O}\left(\mathcal{E}_{T}(\widetilde{\mu})\right)\right) \sigma_{3},
$$

where $\widetilde{\mu}_{0} \in \mathbb{C} \backslash\left(\mathcal{O}_{\tau^{-\frac{1}{3}+\frac{\delta}{2}}}( \pm \alpha) \cup \mathcal{O}_{\tau^{-\frac{2}{3}+\delta}}( \pm \mathrm{i} \sqrt{2} \alpha) \cup\{0, \infty\}\right)$ and the path of integration lies in the corresponding canonical domain,

$$
\mathcal{I}_{\tau}(\widetilde{\mu})=\frac{\mathfrak{p}_{\tau}}{8 \mathrm{i}}\left(\digamma_{\tau}(\widetilde{\mu})-\digamma_{\tau}\left(\widetilde{\mu}_{0}\right)\right)
$$

with

$$
\begin{gathered}
\mathfrak{p}_{\tau}:=\frac{4 \mathrm{i}}{\hat{r}_{0}(\tau)}\left(2\left(1+\hat{u}_{0}(\tau)\right)+\frac{\left(-2+\hat{r}_{0}(\tau)\right)}{1+\hat{u}_{0}(\tau)}\right) \\
\digamma_{\tau}(\xi):=\frac{1}{\sqrt{3}} \ln \left(\left(\frac{\sqrt{3} \sqrt{\xi^{2}+2 \alpha^{2}}-\xi+2 \alpha}{\sqrt{3} \sqrt{\xi^{2}+2 \alpha^{2}}+\xi+2 \alpha}\right)\left(\frac{\xi-\alpha}{\xi+\alpha}\right)\right)+\frac{4}{\sqrt{32+\left(\hat{r}_{0}(\tau)-4\right)^{2}}} \\
\times\left(\operatorname{arctanh}\left(\frac{4 \sqrt{\xi^{2}+2 \alpha^{2}}}{\xi\left(\hat{r}_{0}(\tau)+\sqrt{32+\left(\hat{r}_{0}(\tau)-4\right)^{2}}\right)}\right)-\operatorname{arctanh}\left(\frac{4 \sqrt{\xi^{2}+2 \alpha^{2}}}{\xi\left(\hat{r}_{0}(\tau)-\sqrt{32+\left(\hat{r}_{0}(\tau)-4\right)^{2}}\right)}\right)\right. \\
\left.+\operatorname{arctanh}\left(\frac{8\left(-2+\hat{r}_{0}(\tau)\right) \xi^{2}+\alpha^{2}\left(-2+\hat{r}_{0}(\tau)\right)^{2}+12 \alpha^{2}}{\alpha^{2} \hat{r}_{0}(\tau) \sqrt{32+\left(\hat{r}_{0}(\tau)-4\right)^{2}}}\right)\right)
\end{gathered}
$$


and $\mathcal{E}_{T}(\widetilde{\mu})=\mathcal{E}_{T}^{\natural}(\widetilde{\mu})-\mathcal{E}_{T}^{\natural}\left(\widetilde{\mu}_{0}\right)$, where

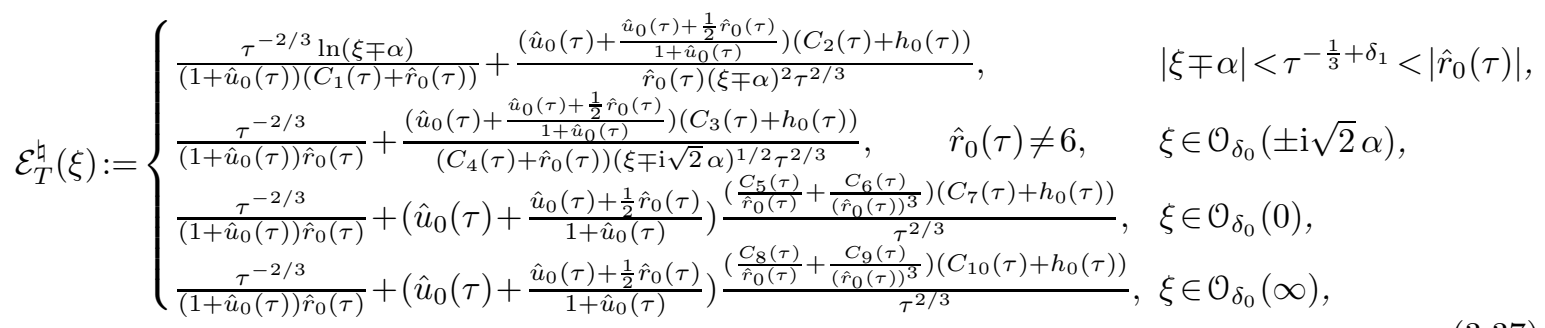

with $10 C_{j}(\tau)={ }_{\tau \rightarrow+\infty} \mathcal{O}(1), j=1, \ldots, 10$.

Proof. From Equation (3.7) and Equations (3.27)-(3.29), one shows that, via Equation (3.10) (cf. Equation (3.21)

$$
2 \mathrm{i} l(\xi) \mathcal{A}_{11}(\xi)+2 l^{2}(\xi) \underset{\tau \rightarrow+\infty}{=} \mathcal{P}_{\infty}(\xi)+\mathcal{P}_{1}(\xi) \Delta_{\tau}(\xi)+\mathcal{O}\left(\xi^{-1} l_{\infty}(\xi) \hat{r}_{0}(\tau)\left(\Delta_{\tau}(\xi)\right)^{2}\right)
$$

where

$$
\begin{gathered}
\mathcal{P}_{\infty}(\xi):=2 l_{\infty}^{2}(\xi)+2 l_{\infty}(\xi)\left(2 \xi+\frac{\alpha^{2}\left(-2+\hat{r}_{0}(\tau)\right)}{\xi}\right) \\
\mathcal{P}_{1}(\xi):=2 l_{\infty}^{2}(\xi)+l_{\infty}(\xi)\left(2 \xi+\frac{\alpha^{2}\left(-2+\hat{r}_{0}(\tau)\right)}{\xi}\right)
\end{gathered}
$$

and, via Equations (3.7), (3.11), and (3.14) (cf. Equation (3.21)),

$$
\mathcal{A}_{12}(\xi) \partial_{\xi} \mathcal{A}_{21}(\xi)-\mathcal{A}_{21}(\xi) \partial_{\xi} \mathcal{A}_{12}(\xi) \underset{\tau \rightarrow+\infty}{=} \frac{2 \mathrm{i} \alpha^{4} \hat{r}_{0}(\tau) \mathfrak{p}_{\tau}}{\xi^{3}}+\mathcal{O}\left(\frac{\tau^{-2 / 3}}{\xi^{3}\left(1+\hat{u}_{0}(\tau)\right)}\right)
$$

where $\mathfrak{p}_{\tau}$ is defined by Equation (3.35). Substituting Equations (3.38) and (3.41) into Equation (3.21) and expanding $\left(2 \mathrm{i} l(\xi) \mathcal{A}_{11}(\xi)+2 l^{2}(\xi)\right)^{-1}$ into a series of powers of $\Delta_{\tau}(\xi)$, one arrives at

$$
\int_{\widetilde{\mu}_{0}}^{\widetilde{\mu}} \operatorname{diag}\left((T(\xi))^{-1} \partial_{\xi} T(\xi)\right) \mathrm{d} \xi \underset{\tau \rightarrow+\infty}{=}\left(\mathcal{I}_{\tau}(\widetilde{\mu})+\mathcal{O}\left(\mathcal{E}_{T}(\widetilde{\mu})\right)\right) \sigma_{3},
$$

where

$$
\mathcal{I}_{\tau}(\widetilde{\mu}):=-\mathrm{i} \alpha^{4} \hat{r}_{0}(\tau) \mathfrak{p}_{\tau} \int_{\widetilde{\mu}_{0}}^{\widetilde{\mu}} \frac{1}{\xi^{3} \mathcal{P}_{\infty}(\xi)} \mathrm{d} \xi
$$

and

$$
\mathcal{E}_{T}(\widetilde{\mu}):=\mathrm{i} \alpha^{4} \hat{r}_{0}(\tau) \mathfrak{p}_{\tau} \int_{\widetilde{\mu}_{0}}^{\widetilde{\mu}} \frac{\mathcal{P}_{1}(\xi) \Delta_{\tau}(\xi)}{\xi^{3}\left(\mathcal{P}_{\infty}(\xi)\right)^{2}} \mathrm{~d} \xi+\frac{\tau^{-2 / 3}}{\left(1+\hat{u}_{0}(\tau)\right)} \int_{\widetilde{\mu}_{0}}^{\widetilde{\mu}} \frac{1}{\xi^{3} \mathcal{P}_{\infty}(\xi)} \mathrm{d} \xi
$$

Via Equation (3.27), it follows from Equation (3.39) that

$$
\frac{1}{\xi^{3} \mathcal{P}_{\infty}(\xi)}=\frac{\xi\left(\xi\left(4 \xi^{2}+2 \alpha^{2}\left(-2+\hat{r}_{0}(\tau)\right)\right)-4\left(\xi^{2}-\alpha^{2}\right)\left(\xi^{2}+2 \alpha^{2}\right)^{1 / 2}\right)}{2\left(\xi^{2}-\alpha^{2}\right)\left(\xi^{2}+2 \alpha^{2}\right)^{1 / 2}\left(\left(\xi\left(4 \xi^{2}+2 \alpha^{2}\left(-2+\hat{r}_{0}(\tau)\right)\right)\right)^{2}-16\left(\xi^{2}-\alpha^{2}\right)^{2}\left(\xi^{2}+2 \alpha^{2}\right)\right)}:
$$

writing the factorization

$$
\begin{aligned}
& \left(\xi\left(4 \xi^{2}+2 \alpha^{2}\left(-2+\hat{r}_{0}(\tau)\right)\right)\right)^{2}-16\left(\xi^{2}-\alpha^{2}\right)^{2}\left(\xi^{2}+2 \alpha^{2}\right) \\
& =16 \alpha^{2}\left(-2+\hat{r}_{0}(\tau)\right)\left(\xi^{4}+\frac{\xi^{2} \alpha^{2}}{\left(-2+\hat{r}_{0}(\tau)\right)}\left(3+\left(\frac{-2+\hat{r}_{0}(\tau)}{2}\right)^{2}\right)-\frac{2 \alpha^{4}}{\left(-2+\hat{r}_{0}(\tau)\right)}\right) \\
& =16 \alpha^{2}\left(-2+\hat{r}_{0}(\tau)\right)\left(\xi^{2}+\hat{z}_{+}\right)\left(\xi^{2}+\hat{z}_{-}\right),
\end{aligned}
$$

where

$$
\hat{z}_{ \pm}:=\frac{\alpha^{2}}{2\left(-2+\hat{r}_{0}(\tau)\right)}\left(3+\left(\frac{-2+\hat{r}_{0}(\tau)}{2}\right)^{2} \mp \frac{\hat{r}_{0}(\tau)}{4} \sqrt{32+\left(\hat{r}_{0}(\tau)-4\right)^{2}}\right)
$$

\footnotetext{
${ }^{10}$ In case $\hat{r}_{0}(\tau) \rightarrow 6$, the factor $(\xi \mp \mathrm{i} \sqrt{2} \alpha)^{1 / 2}$ which appears in the second line of Equation (3.37) should be changed to $\xi \mp \mathrm{i} \sqrt{2} \alpha$.
} 
one shows that, for $\hat{z}_{+} \not \equiv \hat{z}_{-}$,

$$
\begin{aligned}
\mathcal{I}_{\tau}(\widetilde{\mu}) & =-\frac{\mathrm{i} \alpha^{2} \hat{r}_{0}(\tau) \mathfrak{p}_{\tau}}{32\left(-2+\hat{r}_{0}(\tau)\right)}\left(\int_{\widetilde{\mu}_{0}}^{\widetilde{\mu}} \frac{\xi^{2}\left(4 \xi^{2}+2 \alpha^{2}\left(-2+\hat{r}_{0}(\tau)\right)\right) \sqrt{\xi^{2}+2 \alpha^{2}}}{\left(\xi^{2}-\alpha^{2}\right)\left(\xi^{2}+2 \alpha^{2}\right)\left(\xi^{2}+\hat{z}_{+}\right)\left(\xi^{2}+\hat{z}_{-}\right)} \mathrm{d} \xi\right. \\
& \left.-4 \int_{\widetilde{\mu}_{0}}^{\widetilde{\mu}} \frac{\xi}{\left(\xi^{2}+\hat{z}_{+}\right)\left(\xi^{2}+\hat{z}_{-}\right)} \mathrm{d} \xi\right) .
\end{aligned}
$$

Write the partial fraction decomposition

$$
\begin{aligned}
\frac{\xi^{2}\left(4 \xi^{2}+2 \alpha^{2}\left(-2+\hat{r}_{0}(\tau)\right)\right)}{\left(\xi^{2}-\alpha^{2}\right)\left(\xi^{2}+2 \alpha^{2}\right)\left(\xi^{2}+\hat{z}_{+}\right)\left(\xi^{2}+\hat{z}_{-}\right)} & =\frac{A_{0}(\tau)}{\xi-\alpha}+\frac{B_{0}(\tau)}{\xi+\alpha}+\frac{C_{0}(\tau) \xi+D_{0}(\tau)}{\xi^{2}+2 \alpha^{2}}+\frac{E_{0}(\tau) \xi+F_{0}(\tau)}{\xi^{2}+\hat{z}_{+}} \\
& +\frac{G_{0}(\tau) \xi+H_{0}(\tau)}{\xi^{2}+\hat{z}_{-}}:
\end{aligned}
$$

to determine $A_{0}(\tau), \ldots, H_{0}(\tau)$, one notes that the left-hand side of Equation (3.47) is symmetric $(\xi \rightarrow-\xi)$, whence it follows that

$$
A_{0}(\tau)=-B_{0}(\tau), \quad C_{0}(\tau)=E_{0}(\tau)=G_{0}(\tau)=0,
$$

and to determine the remaining coefficients, that is, $A_{0}(\tau), D_{0}(\tau), F_{0}(\tau)$, and $H_{0}(\tau)$, one compares residues (at $\xi=\alpha, \xi^{2}=-2 \alpha^{2}$, and $\xi^{2}=-\hat{z}_{ \pm}$) on the left- and right-hand sides of Equation (3.47) and uses the relations (cf. Equation (3.45) )

$$
\hat{z}_{+}+\hat{z}_{-}=\frac{\alpha^{2}}{\left(-2+\hat{r}_{0}(\tau)\right)}\left(3+\left(\frac{-2+\hat{r}_{0}(\tau)}{2}\right)^{2}\right) \quad \text { and } \quad \hat{z}_{+} \hat{z}_{-}=-\frac{2 \alpha^{4}}{\left(-2+\hat{r}_{0}(\tau)\right)}
$$

to show that

$$
\begin{array}{cc}
A_{0}(\tau)=\frac{\alpha \hat{r}_{0}(\tau)}{3\left(\alpha^{2}+\hat{z}_{+}\right)\left(\alpha^{2}+\hat{z}_{-}\right)}=\frac{4\left(-2+\hat{r}_{0}(\tau)\right)}{3 \alpha^{3} \hat{r}_{0}(\tau)}, & D_{0}(\tau)=\frac{4 \alpha^{2} \hat{r}_{0}(\tau)-24 \alpha^{2}}{3\left(-2 \alpha^{2}+\hat{z}_{-}\right)\left(-2 \alpha^{2}+\hat{z}_{+}\right)}=\frac{8\left(-2+\hat{r}_{0}(\tau)\right)}{3 \alpha^{2}\left(6-\hat{r}_{0}(\tau)\right)}, \\
F_{0}(\tau)=\frac{2 \alpha^{2} \hat{z}_{+}\left(-2+\hat{r}_{0}(\tau)\right)-4\left(\hat{z}_{+}\right)^{2}}{\left(\alpha^{2}+\hat{z}_{+}\right)\left(-2 \alpha^{2}+\hat{z}_{+}\right)\left(\hat{z}_{+}-\hat{z}_{-}\right)}, & H_{0}(\tau)=\frac{4\left(\hat{z}_{-}\right)^{2}-2 \alpha^{2} \hat{z}_{-}\left(-2+\hat{r}_{0}(\tau)\right)}{\left(\alpha^{2}+\hat{z}_{-}\right)\left(-2 \alpha^{2}+\hat{z}_{-}\right)\left(\hat{z}_{+}-\hat{z}_{-}\right)} \Rightarrow \\
F_{0}(\tau)+H_{0}(\tau)=-\frac{16\left(-2+\hat{r}_{0}(\tau)\right)}{\alpha^{2} \hat{r}_{0}(\tau)\left(6-\hat{r}_{0}(\tau)\right)} .
\end{array}
$$

(Note: it is the quantity $F_{0}(\tau)+H_{0}(\tau)$, and not $F_{0}(\tau)$ and $H_{0}(\tau)$ individually, that is requisite for the ensuing calculation.) Substituting Equations (3.49), (3.48), and (3.47) into Equation (3.46), one arrives at, after an integration argument and neglecting $\xi$-independent terms (that is, with abuse of nomenclature, "constants of integration"),

$$
\begin{aligned}
\mathcal{I}_{\tau}(\widetilde{\mu}) & =-\frac{\mathrm{i} \alpha^{2} \hat{r}_{0}(\tau) \mathfrak{p}_{\tau}}{32\left(-2+\hat{r}_{0}(\tau)\right)}\left(\left(2 \alpha A_{0}(\tau)+D_{0}(\tau)+\frac{8\left(-2+\hat{r}_{0}(\tau)\right)}{\alpha^{2} \hat{r}_{0}(\tau)\left(\hat{r}_{0}(\tau)-6\right)}\right) \ln \left(\sqrt{\xi^{2}+2 \alpha^{2}}+\xi\right)\right. \\
& -\frac{8\left(-2+\hat{r}_{0}(\tau)\right)}{\alpha^{2} \hat{r}_{0}(\tau)\left(\hat{r}_{0}(\tau)-6\right)} \ln \left(\sqrt{\xi^{2}+2 \alpha^{2}}-\xi\right)+\sqrt{3} \alpha A_{0}(\tau) \ln \left(\left(\frac{\sqrt{3} \sqrt{\xi^{2}+2 \alpha^{2}}-\xi+2 \alpha}{\sqrt{3} \sqrt{\xi^{2}+2 \alpha^{2}}+\xi+2 \alpha}\right)\left(\frac{\xi-\alpha}{\xi+\alpha}\right)\right) \\
& +\frac{16\left(-2+\hat{r}_{0}(\tau)\right)}{\alpha^{2} \hat{r}_{0}(\tau) \sqrt{32+\left(\hat{r}_{0}(\tau)-4\right)^{2}}}\left(\operatorname{arctanh}\left(\frac{8\left(-2+\hat{r}_{0}(\tau)\right) \xi^{2}+\alpha^{2}\left(-2+\hat{r}_{0}(\tau)\right)^{2}+12 \alpha^{2}}{\alpha^{2} \hat{r}_{0}(\tau) \sqrt{32+\left(\hat{r}_{0}(\tau)-4\right)^{2}}}\right)\right. \\
& \left.+\operatorname{arctanh}\left(\frac{4 \sqrt{\xi^{2}+2 \alpha^{2}}}{\xi\left(\hat{r}_{0}(\tau)+\sqrt{32+\left(\hat{r}_{0}(\tau)-4\right)^{2}}\right)}\right)-\operatorname{arctanh}\left(\frac{4 \sqrt{\xi^{2}+2 \alpha^{2}}}{\xi\left(\hat{r}_{0}(\tau)-\sqrt{32+\left(\hat{r}_{0}(\tau)-4\right)^{2}}\right)}\right)\right)\left.\right|_{\widetilde{\mu}_{0}} ^{\widetilde{\mu}}
\end{aligned}
$$

From Equations (3.49), one notes that

$$
2 \alpha A_{0}(\tau)+D_{0}(\tau)+\frac{16\left(-2+\hat{r}_{0}(\tau)\right)}{\alpha^{2} \hat{r}_{0}(\tau)\left(\hat{r}_{0}(\tau)-6\right)}=0
$$


whence (cf. Equation (3.50)

$$
\begin{gathered}
\left(2 \alpha A_{0}(\tau)+D_{0}(\tau)+\frac{8\left(-2+\hat{r}_{0}(\tau)\right)}{\alpha^{2} \hat{r}_{0}(\tau)\left(\hat{r}_{0}(\tau)-6\right)}\right) \ln \left(\sqrt{\xi^{2}+2 \alpha^{2}}+\xi\right)-\frac{8\left(-2+\hat{r}_{0}(\tau)\right)}{\alpha^{2} \hat{r}_{0}(\tau)\left(\hat{r}_{0}(\tau)-6\right)} \ln \left(\sqrt{\xi^{2}+2 \alpha^{2}}-\xi\right) \\
=-\frac{8\left(-2+\hat{r}_{0}(\tau)\right)}{\alpha^{2} \hat{r}_{0}(\tau)\left(\hat{r}_{0}(\tau)-6\right)} \ln \left(2 \alpha^{2}\right),
\end{gathered}
$$

which is a $\xi$-independent term (a "constant of integration"): neglecting this term, one arrives at (after simplification) Equations (3.34)-(3.36).

One now studies the error term $\mathcal{O}\left(\mathcal{E}_{T}(\widetilde{\mu})\right)$ defined by Equation (3.43). Recall the expression for $\left(\xi^{3} \mathcal{P}_{\infty}(\xi)\right)^{-1}$ given in Equation (3.44): from this latter expression, and those for $\Delta_{\tau}(\xi)$ (cf. Equation $(\underline{3.28})$ ) and $\mathcal{P}_{1}(\xi)$ (cf. Equation (3.40) $)$, one shows that

$$
\frac{\mathcal{P}_{1}(\xi) \Delta_{\tau}(\xi)}{\xi^{3}\left(\mathcal{P}_{\infty}(\xi)\right)^{2}}=\frac{\xi^{2}\left(h_{0}(\tau)+\xi^{2}\left(a-\frac{i}{2}\right)\right)\left(2 \xi l_{\infty}(\xi)+2 \xi^{2}+\alpha^{2}\left(-2+\hat{r}_{0}(\tau)\right)\right) \tau^{-2 / 3}}{8\left(\xi^{2}-\alpha^{2}\right)^{3}\left(\xi^{2}+2 \alpha^{2}\right)^{3 / 2}\left(\xi l_{\infty}(\xi)+2 \xi^{2}+\alpha^{2}\left(-2+\hat{r}_{0}(\tau)\right)\right)^{2}}
$$

Using Equation (3.51), one evaluates the first integral in Equation (3.43) explicitly (as done above, for the second integral in Equation (3.43)): the resulting expression for $\mathcal{E}_{T}(\widetilde{\mu})$ is quite cumbersome; therefore, only its asymptotics at the singular and turning points are defined by Equation (3.37).

Corollary 3.2.3. Let $\widetilde{\mu}_{0}$ be defined as in Corollary 3.2 .2 , that is, $\widetilde{\mu}_{0}=\alpha+\tau^{-1 / 3} \widetilde{\Lambda}$, where $\widetilde{\Lambda}={ }_{\tau \rightarrow+\infty}$ $\mathcal{O}\left(\tau^{\varepsilon}\right)$, and $\delta, \delta_{1}$ be defined in conditions (3.12). Then, for $0<\delta<\varepsilon<1 / 9$ and $\varepsilon<\delta_{1}<1 / 3$,

$$
\int_{\widetilde{\mu}_{0}}^{\widetilde{\mu}} \operatorname{diag}\left((T(\xi))^{-1} \partial_{\xi} T(\xi)\right) \mathrm{d} \xi \underset{\tau \rightarrow+\infty}{=}\left(\frac{\mathfrak{p}_{\tau}}{8 \mathrm{i}}\left(\digamma_{\tau}(\widetilde{\mu})-\digamma_{\tau}^{\sharp}\right)+\mathcal{O}\left(\mathcal{E}_{T}^{\natural}(\widetilde{\mu})\right)+\mathcal{O}\left(\tau^{-2(\varepsilon-\delta)}\right)+\mathcal{O}\left(\tau^{-\frac{1}{3}+\varepsilon+\delta}\right)\right) \sigma_{3},
$$

where $\mathfrak{p}_{\tau}, \digamma_{\tau}(\widetilde{\mu})$, and $\mathcal{E}_{T}^{\natural}(\widetilde{\mu})$ are defined by Equations (3.35), (3.36), and (3.37), respectively, and

$$
\begin{aligned}
\digamma_{\tau}^{\sharp} & :=\mp \frac{1}{\sqrt{3}} \ln (3 \alpha)+\frac{2}{\sqrt{32+\left(\hat{r}_{0}(\tau)-4\right)^{2}}} \ln \left(\frac{\hat{r}_{0}(\tau)+4+\sqrt{32+\left(\hat{r}_{0}(\tau)-4\right)^{2}}}{-\hat{r}_{0}(\tau)-4+\sqrt{32+\left(\hat{r}_{0}(\tau)-4\right)^{2}}}\right) \\
& \mp \frac{2}{\sqrt{32+\left(\hat{r}_{0}(\tau)-4\right)^{2}}} \ln \left(\left(\frac{\hat{r}_{0}(\tau)+4 \sqrt{3}-\sqrt{32+\left(\hat{r}_{0}(\tau)-4\right)^{2}}}{\hat{r}_{0}(\tau)+4 \sqrt{3}+\sqrt{32+\left(\hat{r}_{0}(\tau)-4\right)^{2}}}\right)\right. \\
& \left.\times\left(\frac{\hat{r}_{0}(\tau)-4 \sqrt{3}+\sqrt{32+\left(\hat{r}_{0}(\tau)-4\right)^{2}}}{\hat{r}_{0}(\tau)-4 \sqrt{3}-\sqrt{32+\left(\hat{r}_{0}(\tau)-4\right)^{2}}}\right)\right) \pm \frac{1}{\sqrt{3}}\left(-\frac{1}{3} \ln \tau+\ln \widetilde{\Lambda}\right),
\end{aligned}
$$

with the upper (resp., lower) signs taken if the positive $(+)$ (resp., negative (-)) branch of the squareroot function $\sqrt{\xi^{2}+2 \alpha^{2}}$ is chosen.

Proof. Substituting $\widetilde{\mu}_{0}$ as the argument of the functions $\digamma_{\tau}(\xi)$ and $\mathcal{E}_{T}^{\natural}(\xi)$ (cf. Equation (3.36) and the first line of Equation (3.37), respectively) and expanding with respect to (the small parameter) $\tau^{-1 / 3} \widetilde{\Lambda}$, one arrives at the following estimates:

$$
\digamma_{\tau}\left(\widetilde{\mu}_{0}\right) \underset{\tau \rightarrow+\infty}{=} \digamma_{\tau}^{\sharp}+\mathcal{O}\left(\tau^{-1 / 3} \widetilde{\Lambda}\right),
$$

and

$$
\mathcal{E}_{T}^{\natural}\left(\widetilde{\mu}_{0}\right) \underset{\tau \rightarrow+\infty}{=} \mathcal{O}\left(\frac{\tau^{-2 / 3} \ln \tau}{\left(1+\hat{u}_{0}(\tau)\right)\left(C_{1}(\tau)+\hat{r}_{0}(\tau)\right)}\right)+\mathcal{O}\left(\frac{\left(2 \hat{u}_{0}(\tau)+\frac{1}{2} \hat{r}_{0}(\tau)\right)\left(C_{2}(\tau)+h_{0}(\tau)\right)}{\hat{r}_{0}(\tau) \widetilde{\Lambda}^{2}}\right),
$$

where $\digamma_{\tau}^{\sharp}$ is defined by Equation (3.53), and $C_{j}(\tau)={ }_{\tau \rightarrow+\infty} \mathcal{O}(1), j=1,2$. Demanding that the above error estimates are decaying, and using the definition of the parameters $\delta, \delta_{1}$ given in conditions (3.12) for the functions $\hat{r}_{0}(\tau), \hat{u}_{0}(\tau)$, and $h_{0}(\tau)$, one arrives at the inequalities stated in the Corollary.

Corollary 3.2.4. Under the conditions of Corollary 3.2 .2 , for the branch of $l(\xi)$ that is positive for large and small positive $\xi$,

$$
-\mathrm{i} \tau^{2 / 3} \int_{\widetilde{\mu}_{0}}^{\widetilde{\mu}} l(\xi) \mathrm{d} \xi \underset{\substack{\tau \rightarrow+\infty \\ \operatorname{Re}(\vec{\mu}) \rightarrow+\infty}}{=}-\mathrm{i}\left(\tau^{2 / 3} \widetilde{\mu}^{2}+\left(a-\frac{\mathrm{i}}{2}\right) \ln \widetilde{\mu}\right)+\mathrm{i} \tau^{2 / 3} 3(\sqrt{3}-1) \alpha^{2}+\mathrm{i} 2 \sqrt{3} \widetilde{\Lambda}^{2}
$$




$$
\begin{aligned}
& -\frac{\mathrm{i}}{2 \sqrt{3}}\left(\left(a-\frac{\mathrm{i}}{2}\right)+\frac{h_{0}(\tau)}{\alpha^{2}}\right)\left(\frac{1}{3} \ln \tau-\ln \tilde{\Lambda}\right)+C_{\infty}^{\mathrm{WKB}}+\mathcal{O}\left(\tau^{-2(\varepsilon-\delta)}\right) \\
& +\mathcal{O}\left(\tau^{-\frac{1}{3}+3 \varepsilon}\right),
\end{aligned}
$$

where

$$
C_{\infty}^{\mathrm{WKB}}:=\mathrm{i}\left(a-\frac{\mathrm{i}}{2}\right) \ln \left(\frac{(\sqrt{3}+1) \alpha}{2}\right)-\frac{\mathrm{i}}{2 \sqrt{3}}\left(\left(a-\frac{\mathrm{i}}{2}\right)+\frac{h_{0}(\tau)}{\alpha^{2}}\right) \ln \left(\frac{6 \alpha}{(\sqrt{3}+1)^{2}}\right),
$$

and

$$
\begin{gathered}
-\mathrm{i} \tau^{2 / 3} \int_{\widetilde{\mu}_{0}}^{\widetilde{\mu}} l(\xi) \mathrm{d} \xi \underset{\substack{\tau \rightarrow+\infty \\
\operatorname{Re}(\widetilde{\mu}) \rightarrow+0}}{=} \frac{\mathrm{i} \tau^{2 / 3} 2 \sqrt{2} \alpha^{3}}{\widetilde{\mu}}-\mathrm{i} \tau^{2 / 3} 3 \sqrt{3} \alpha^{2}-\mathrm{i} 2 \sqrt{3} \widetilde{\Lambda}^{2}+\frac{\mathrm{i}}{2 \sqrt{3}}\left(\left(a-\frac{\mathrm{i}}{2}\right)+\frac{h_{0}(\tau)}{\alpha^{2}}\right) \\
\times\left(\frac{1}{3} \ln \tau-\ln \widetilde{\Lambda}\right)+C_{0}^{\mathrm{WKB}}+\mathcal{O}\left(\tau^{-2(\varepsilon-\delta)}\right)+\mathcal{O}\left(\tau^{-\frac{1}{3}+3 \varepsilon}\right),
\end{gathered}
$$

where

$$
C_{0}^{\mathrm{WKB}}:=-\mathrm{i}\left(a-\frac{\mathrm{i}}{2}\right) \ln \left(\frac{\sqrt{3}+1}{\sqrt{2}}\right)+\frac{\mathrm{i}}{2 \sqrt{3}}\left(\left(a-\frac{\mathrm{i}}{2}\right)+\frac{h_{0}(\tau)}{\alpha^{2}}\right) \ln \left(3 \alpha \mathrm{e}^{-\mathrm{i} \pi}\right) .
$$

Proof. Follows from Corollary 3.2.2. Equation (3.31), by choosing the corresponding branches in Equations (3.25) and (3.32) and taking the limits $\operatorname{Re}(\widetilde{\mu}) \rightarrow+\infty$ and $\operatorname{Re}(\widetilde{\mu}) \rightarrow+0$ : the error estimate $\mathcal{O}\left(\mathcal{E}_{l}^{\natural}(\xi)\right)$ in Equation (3.31) is defined by Equation (3.26).

Corollary 3.2.5. Under the conditions of Corollary 3.2.3, for the branch of $l(\xi)$ that is positive for large and small positive $\xi$,

$$
\begin{aligned}
\int_{\widetilde{\mu}_{0}}^{\widetilde{\mu}} \operatorname{diag}\left((T(\xi))^{-1} \partial_{\xi} T(\xi)\right) \mathrm{d} \xi \underset{\substack{\tau \rightarrow+\infty \\
\operatorname{Re}(\widetilde{\mu}) \rightarrow+\infty}}{=} & \left(-\frac{\sqrt{32+\left(\hat{r}_{0}(\tau)-4\right)^{2}}}{8 \sqrt{3}}\left(-\frac{1}{3} \ln \tau+\ln \widetilde{\Lambda}\right)+\mathcal{I}_{\infty}^{\sharp}(\tau)\right. \\
& +\mathcal{O}\left(\frac{\hat{r}_{0}(\tau)}{\widetilde{\mu}^{2}}\right)+\mathcal{O}\left(\tau^{\delta-2 \delta_{1}} \ln \tau\right)+\mathcal{O}\left(\tau^{-2(\varepsilon-\delta)}\right) \\
& \left.+\mathcal{O}\left(\tau^{-\frac{2}{3}+2 \delta}\right)+\mathcal{O}\left(\tau^{-\frac{1}{3}+\varepsilon+\delta}\right)\right) \sigma_{3},
\end{aligned}
$$

where

$$
\begin{aligned}
\mathcal{I}_{\infty}^{\sharp}(\tau): & =\frac{1}{4} \ln \left(\left(\frac{\hat{r}_{0}(\tau)+4 \sqrt{3}-\sqrt{32+\left(\hat{r}_{0}(\tau)-4\right)^{2}}}{\hat{r}_{0}(\tau)+4 \sqrt{3}+\sqrt{32+\left(\hat{r}_{0}(\tau)-4\right)^{2}}}\right)\left(\frac{\hat{r}_{0}(\tau)-4 \sqrt{3}+\sqrt{32+\left(\hat{r}_{0}(\tau)-4\right)^{2}}}{\hat{r}_{0}(\tau)-4 \sqrt{3}-\sqrt{32+\left(\hat{r}_{0}(\tau)-4\right)^{2}}}\right)\right) \\
& +\frac{\sqrt{32+\left(\hat{r}_{0}(\tau)-4\right)^{2}}}{8 \sqrt{3}} \ln \left(\frac{6 \alpha}{(\sqrt{3}+1)^{2}}\right)-\frac{1}{2} \ln \left(\frac{\hat{u}_{0}(\tau)}{\hat{r}_{0}(\tau)}\right)-\frac{3}{4} \ln 2-\frac{\mathrm{i} \pi}{4},
\end{aligned}
$$

and

$$
\begin{aligned}
\int_{\widetilde{\mu}_{0}}^{\widetilde{\mu}} \operatorname{diag}\left((T(\xi))^{-1} \partial_{\xi} T(\xi)\right) \mathrm{d} \xi \underset{\substack{\tau \rightarrow+\infty \\
\operatorname{Re}(\widetilde{\mu}) \rightarrow+0}}{ }\left(\frac{\sqrt{32+\left(\hat{r}_{0}(\tau)-4\right)^{2}}}{8 \sqrt{3}}\left(-\frac{1}{3} \ln \tau+\ln \widetilde{\Lambda}\right)+\mathcal{I}_{0}^{\sharp}(\tau)\right. \\
+\mathcal{O}\left(\hat{r}_{0}(\tau) \widetilde{\mu}\right)+\mathcal{O}\left(\tau^{\delta-2 \delta_{1}} \ln \tau\right)+\mathcal{O}\left(\tau^{-2(\varepsilon-\delta)}\right) \\
\left.+\mathcal{O}\left(\tau^{-\frac{2}{3}+2 \delta}\right)+\mathcal{O}\left(\tau^{-\frac{1}{3}+\varepsilon+\delta}\right)+\mathcal{O}\left(\tau^{-\frac{1}{3}+\delta-\delta_{1}}\right)\right) \sigma_{3},
\end{aligned}
$$

where

$$
\begin{aligned}
\mathcal{I}_{0}^{\sharp}(\tau): & =-\frac{1}{4} \ln \left(\left(\frac{\hat{r}_{0}(\tau)+4 \sqrt{3}-\sqrt{32+\left(\hat{r}_{0}(\tau)-4\right)^{2}}}{\hat{r}_{0}(\tau)+4 \sqrt{3}+\sqrt{32+\left(\hat{r}_{0}(\tau)-4\right)^{2}}}\right)\left(\frac{\hat{r}_{0}(\tau)-4 \sqrt{3}+\sqrt{32+\left(\hat{r}_{0}(\tau)-4\right)^{2}}}{\hat{r}_{0}(\tau)-4 \sqrt{3}-\sqrt{32+\left(\hat{r}_{0}(\tau)-4\right)^{2}}}\right)\right) \\
& -\frac{\sqrt{32+\left(\hat{r}_{0}(\tau)-4\right)^{2}}}{8 \sqrt{3}} \ln \left(3 \alpha \mathrm{e}^{-\mathrm{i} \pi}\right)+\frac{1}{2} \ln \left(\frac{\hat{u}_{0}(\tau)}{\hat{r}_{0}(\tau)}\right)+\frac{3}{4} \ln 2+\frac{\mathrm{i} \pi}{4} .
\end{aligned}
$$


Proof. Taking the limit $\operatorname{Re}(\widetilde{\mu}) \rightarrow+\infty$ in Equation (3.52), with $\digamma_{\tau}(\xi)$ and $\mathcal{E}_{T}^{\natural}(\xi)$ defined by Equations (3.36) and (3.37), respectively, and using conditions (3.12), one arrives at

$$
\begin{aligned}
\digamma_{\tau}(\widetilde{\mu}) \underset{\substack{\tau \rightarrow \overrightarrow{+}+\infty \\
\operatorname{Re}(\widetilde{\mu}) \rightarrow+\infty}}{=} \frac{1}{\sqrt{3}} \ln \left(\frac{\sqrt{3}-1}{\sqrt{3}+1}\right)-\frac{2}{\sqrt{32+\left(\hat{r}_{0}(\tau)-4\right)^{2}}} \ln \left(\left(\frac{\hat{r}_{0}(\tau)-4+\sqrt{32+\left(\hat{r}_{0}(\tau)-4\right)^{2}}}{\hat{r}_{0}(\tau)-4-\sqrt{32+\left(\hat{r}_{0}(\tau)-4\right)^{2}}}\right)\right. \\
\left.\times\left(\frac{-\hat{r}_{0}(\tau)-4+\sqrt{32+\left(\hat{r}_{0}(\tau)-4\right)^{2}}}{\hat{r}_{0}(\tau)+4+\sqrt{32+\left(\hat{r}_{0}(\tau)-4\right)^{2}}}\right)\right)+\mathcal{O}\left(\widetilde{\mu}^{-2}\right),
\end{aligned}
$$

and

$$
\mathcal{E}_{T}^{\natural}(\widetilde{\mu}) \underset{\substack{\tau \rightarrow \overrightarrow{\widetilde{\mu}}) \rightarrow+\infty \\ \operatorname{Re}}}{=} \mathcal{O}\left(\tau^{-2 \delta_{1}}\right)+\mathcal{O}\left(\tau^{-\frac{2}{3}+2 \delta}\right) .
$$

One now computes asymptotics of $\mathfrak{p}_{\tau}$ (cf. Equation (3.35)). Using conditions (3.12) and Equation (3.16), one shows that

$$
\frac{-2+\hat{r}_{0}(\tau)}{1+\hat{u}_{0}(\tau)} \underset{\tau \rightarrow+\infty}{=} \frac{\hat{r}_{0}(\tau)\left(4-\hat{r}_{0}(\tau)\right)}{4}+2\left(1+\hat{u}_{0}(\tau)\right)-4+\mathcal{O}\left(\tau^{-2 / 3} \varkappa_{0}^{2}(\tau)\right),
$$

where

$$
\varkappa_{0}^{2}(\tau):=\frac{4}{\alpha^{2}}\left(h_{0}(\tau)+\frac{\alpha^{2}\left(a-\frac{\mathrm{i}}{2}\right)}{1+\hat{u}_{0}(\tau)}\right),
$$

whence, substituting Equation (3.64) into Equation (3.35), one obtains

$$
\mathfrak{p}_{\tau} \underset{\tau \rightarrow+\infty}{=} \frac{4 \mathrm{i}}{\hat{r}_{0}(\tau)}\left(4 \hat{u}_{0}(\tau)+\frac{\hat{r}_{0}(\tau)\left(4-\hat{r}_{0}(\tau)\right)}{4}+\mathcal{O}\left(\tau^{-2 / 3} \varkappa_{0}^{2}(\tau)\right)\right) .
$$

Solving Equation (3.16) for $\hat{u}_{0}(\tau)$ and taking into account conditions (3.12), one deduces that

$$
16 \hat{u}_{0}(\tau) \underset{\tau \rightarrow+\infty}{=}-\hat{r}_{0}(\tau)\left(4-\hat{r}_{0}(\tau)\right)+\hat{r}_{0}(\tau) \sqrt{32+\left(\hat{r}_{0}(\tau)-4\right)^{2}}\left(1+\mathcal{O}\left(\frac{\tau^{-2 / 3} \varkappa_{0}^{2}(\tau)}{\left(\hat{r}_{0}(\tau)\right)^{2}}\right)\right) .
$$

Now, substituting Equation (3.67) into Equation (3.66), one arrives at

$$
\mathfrak{p}_{\tau} \underset{\tau \rightarrow+\infty}{=} \mathrm{i} \sqrt{32+\left(\hat{r}_{0}(\tau)-4\right)^{2}}\left(1+\mathcal{O}\left(\frac{\tau^{-2 / 3} \varkappa_{0}^{2}(\tau)}{\left(\hat{r}_{0}(\tau)\right)^{2}}\right)\right) .
$$

Substituting the expansions (3.62), (3.63), and (3.68) into Equation (3.52) (with the upper signs in the formula for $\digamma_{\tau}^{\sharp}$ taken (cf. Equation (3.53)), and taking into account conditions (3.12), one shows that

$$
\begin{gathered}
\int_{\widetilde{\mu}_{0}}^{\widetilde{\mu}} \operatorname{diag}\left((T(\xi))^{-1} \partial_{\xi} T(\xi)\right) \mathrm{d} \xi \underset{\substack{\tau \rightarrow+\infty \\
\operatorname{Re}(\widetilde{\mu}) \rightarrow+\infty}}{=}\left(\mathcal{I}_{\infty}(\tau)+\mathcal{O}\left(\tau^{\delta-2 \delta_{1}} \ln \tau\right)+\mathcal{O}\left(\tau^{-2(\varepsilon-\delta)}\right)+\mathcal{O}\left(\tau^{-\frac{1}{3}+\varepsilon+\delta}\right)\right. \\
\left.+\mathcal{O}\left(\tau^{-\frac{2}{3}+2 \delta}\right)+\mathcal{O}\left(\hat{r}_{0}(\tau) \widetilde{\mu}^{-2}\right)\right) \sigma_{3}
\end{gathered}
$$

where

$$
\mathcal{I}_{\infty}(\tau)=-\frac{\sqrt{32+\left(\hat{r}_{0}(\tau)-4\right)^{2}}}{8 \sqrt{3}}\left(-\frac{1}{3} \ln \tau+\ln \tilde{\Lambda}\right)+\widehat{\mathcal{I}}_{\infty}(\tau),
$$

with

$$
\begin{aligned}
\widehat{\mathcal{I}}_{\infty}(\tau): & =\frac{\sqrt{32+\left(\hat{r}_{0}(\tau)-4\right)^{2}}}{8 \sqrt{3}} \ln \left(\frac{6 \alpha}{(\sqrt{3}+1)^{2}}\right)-\frac{1}{4} \ln \left(\frac{\hat{r}_{0}(\tau)-4+\sqrt{32+\left(\hat{r}_{0}(\tau)-4\right)^{2}}}{\hat{r}_{0}(\tau)-4-\sqrt{32+\left(\hat{r}_{0}(\tau)-4\right)^{2}}}\right) \\
& +\frac{1}{4} \ln \left(\left(\frac{\hat{r}_{0}(\tau)+4 \sqrt{3}-\sqrt{32+\left(\hat{r}_{0}(\tau)-4\right)^{2}}}{\hat{r}_{0}(\tau)+4 \sqrt{3}+\sqrt{32+\left(\hat{r}_{0}(\tau)-4\right)^{2}}}\right)\left(\frac{\hat{r}_{0}(\tau)-4 \sqrt{3}+\sqrt{32+\left(\hat{r}_{0}(\tau)-4\right)^{2}}}{\hat{r}_{0}(\tau)-4 \sqrt{3}-\sqrt{32+\left(\hat{r}_{0}(\tau)-4\right)^{2}}}\right)\right) .
\end{aligned}
$$


Taking the limit $\operatorname{Re}(\widetilde{\mu}) \rightarrow+0$ in Equation (3.52), with $\digamma_{\tau}(\xi)$ and $\mathcal{E}_{T}^{\natural}(\xi)$ defined by Equations (3.36) and (3.37), respectively, and using conditions (3.12), one obtains

$$
\begin{aligned}
\digamma_{\tau}(\widetilde{\mu}) \underset{\substack{\tau \rightarrow+\infty \\
\operatorname{Re}(\widetilde{\mu}) \rightarrow+\infty}}{=} \frac{2}{\sqrt{32+\left(\hat{r}_{0}(\tau)-4\right)^{2}}} \ln \left(\frac{\hat{r}_{0}(\tau) \sqrt{32+\left(\hat{r}_{0}(\tau)-4\right)^{2}}+\left(\left(\hat{r}_{0}(\tau)\right)^{2}-4 \hat{r}_{0}(\tau)+16\right)}{\hat{r}_{0}(\tau) \sqrt{32+\left(\hat{r}_{0}(\tau)-4\right)^{2}}-\left(\left(\hat{r}_{0}(\tau)\right)^{2}-4 \hat{r}_{0}(\tau)+16\right)}\right) \\
+\frac{1}{\sqrt{3}} \ln \left(\mathrm{e}^{\mathrm{i} \pi}\right)+\mathcal{O}(\widetilde{\mu}),
\end{aligned}
$$

and

$$
\mathcal{E}_{T}^{\natural}(\widetilde{\mu}) \underset{\substack{\tau \rightarrow(\vec{\mu}) \rightarrow+\infty \\ \operatorname{Re}(\underset{\mu}{\infty})}}{=} \mathcal{O}\left(\tau^{-2 \delta_{1}}\right)+\mathcal{O}\left(\tau^{-\frac{2}{3}+2 \delta}\right),
$$

whence, substituting the expansions (3.72), (3.73), and (3.68) into Equation (3.52) (with the lower signs in the formula for $\digamma_{\tau}^{\sharp}$ taken (cf. Equation (3.53)), and taking into account conditions (3.12), one arrives at

$$
\begin{gathered}
\int_{\widetilde{\mu}_{0}}^{\widetilde{\mu}} \operatorname{diag}\left((T(\xi))^{-1} \partial_{\xi} T(\xi)\right) \mathrm{d} \xi \underset{\substack{\tau \rightarrow+\infty \\
\operatorname{Re}(\widetilde{\mu}) \rightarrow+\infty}}{=}\left(\mathcal{I}_{0}(\tau)+\mathcal{O}\left(\tau^{\delta-2 \delta_{1}} \ln \tau\right)+\mathcal{O}\left(\tau^{-2(\varepsilon-\delta)}\right)+\mathcal{O}\left(\tau^{-\frac{1}{3}+\varepsilon+\delta}\right)\right. \\
\left.+\mathcal{O}\left(\tau^{-\frac{2}{3}+2 \delta}\right)+\mathcal{O}\left(\hat{r}_{0}(\tau) \widetilde{\mu}\right)\right) \sigma_{3}
\end{gathered}
$$

where

$$
\mathcal{I}_{0}(\tau)=\frac{\sqrt{32+\left(\hat{r}_{0}(\tau)-4\right)^{2}}}{8 \sqrt{3}}\left(-\frac{1}{3} \ln \tau+\ln \tilde{\Lambda}\right)+\widehat{\mathcal{I}}_{0}(\tau),
$$

with

$$
\begin{aligned}
\widehat{\mathcal{I}}_{0}(\tau): & =-\frac{\sqrt{32+\left(\hat{r}_{0}(\tau)-4\right)^{2}}}{8 \sqrt{3}} \ln \left(3 \alpha \mathrm{e}^{-\mathrm{i} \pi}\right)-\frac{1}{4} \ln \left(\frac{\hat{r}_{0}(\tau)+4+\sqrt{32+\left(\hat{r}_{0}(\tau)-4\right)^{2}}}{-\hat{r}_{0}(\tau)-4+\sqrt{32+\left(\hat{r}_{0}(\tau)-4\right)^{2}}}\right) \\
& -\frac{1}{4} \ln \left(\left(\frac{\hat{r}_{0}(\tau)+4 \sqrt{3}-\sqrt{32+\left(\hat{r}_{0}(\tau)-4\right)^{2}}}{\hat{r}_{0}(\tau)+4 \sqrt{3}+\sqrt{32+\left(\hat{r}_{0}(\tau)-4\right)^{2}}}\right)\left(\frac{\hat{r}_{0}(\tau)-4 \sqrt{3}+\sqrt{32+\left(\hat{r}_{0}(\tau)-4\right)^{2}}}{\hat{r}_{0}(\tau)-4 \sqrt{3}-\sqrt{32+\left(\hat{r}_{0}(\tau)-4\right)^{2}}}\right)\right) \\
& +\frac{1}{4} \ln \left(\frac{\hat{r}_{0}(\tau) \sqrt{32+\left(\hat{r}_{0}(\tau)-4\right)^{2}}+\left(\left(\hat{r}_{0}(\tau)\right)^{2}-4 \hat{r}_{0}(\tau)+16\right)}{\hat{r}_{0}(\tau) \sqrt{32+\left(\hat{r}_{0}(\tau)-4\right)^{2}}-\left(\left(\hat{r}_{0}(\tau)\right)^{2}-4 \hat{r}_{0}(\tau)+16\right)}\right) .
\end{aligned}
$$

One now simplifies Equations (3.71) and (3.76); in order to do so, however, several estimates are necessary. Rewrite Equation (3.16) as follows:

$$
\frac{\left(\hat{u}_{0}(\tau)\right)^{2}}{1+\hat{u}_{0}(\tau)}+\frac{\hat{r}_{0}(\tau) \hat{u}_{0}(\tau)}{2\left(1+\hat{u}_{0}(\tau)\right)}-\frac{\left(\hat{r}_{0}(\tau)\right)^{2}}{8}=-\frac{\tau^{-2 / 3} \varkappa_{0}^{2}(\tau)}{8 \alpha^{2}} .
$$

Via Equations (3.35) and (3.68), using Equation (3.77) to eliminate $\left(\hat{u}_{0}(\tau)\right)^{2}$, and taking into account conditions (3.12), one arrives at, after simplification,

$$
\begin{aligned}
\hat{r}_{0}(\tau) \sqrt{32+\left(\hat{r}_{0}(\tau)-4\right)^{2}}-\left(\left(\hat{r}_{0}(\tau)\right)^{2}-4 \hat{r}_{0}(\tau)+16\right) \underset{\tau \rightarrow+\infty}{=} & \frac{8\left(-2+\hat{r}_{0}(\tau)\right)}{1+\hat{u}_{0}(\tau)}\left(1+\mathcal{O}\left(\tau^{-\frac{2}{3}+\delta}\right)\right. \\
& \left.+\mathcal{O}\left(\tau^{-\frac{1}{3}+\delta-\delta_{1}}\right)\right) .
\end{aligned}
$$

The estimates (3.79) - below are derived analogously:

$$
\begin{gathered}
\hat{r}_{0}(\tau) \sqrt{32+\left(\hat{r}_{0}(\tau)-4\right)^{2}}+\left(\left(\hat{r}_{0}(\tau)\right)^{2}-4 \hat{r}_{0}(\tau)+16\right) \underset{\tau \rightarrow+\infty}{=} \\
\left.+\mathcal{O}\left(\tau^{-\frac{1}{3}+\delta-\delta_{1}}\right)\right), \\
\hat{r}_{0}(\tau)+4+\sqrt{32+\left(\hat{r}_{0}(\tau)-4\right)^{2}} \underset{\tau \rightarrow+\infty}{=} \frac{2 \hat{r}_{0}(\tau)\left(1+\hat{u}_{0}(\tau)\right)}{\hat{u}_{0}(\tau)}\left(1+\mathcal{O}\left(\tau^{-\frac{2}{3}-\delta}\right)+\mathcal{O}\left(\tau^{\delta-2 \delta_{1}}\right)\right),
\end{gathered}
$$




$$
\begin{aligned}
\hat{r}_{0}(\tau)+4 & -\sqrt{32+\left(\hat{r}_{0}(\tau)-4\right)^{2}} \underset{\tau \rightarrow+\infty}{=} \frac{8 \hat{u}_{0}(\tau)\left(-2+\hat{r}_{0}(\tau)\right)}{\hat{r}_{0}(\tau)\left(1+\hat{u}_{0}(\tau)\right)}\left(1+\mathcal{O}\left(\tau^{-\frac{2}{3}-\delta}\right)+\mathcal{O}\left(\tau^{\delta-2 \delta_{1}}\right)\right) \\
& \hat{r}_{0}(\tau)-4+\sqrt{32+\left(\hat{r}_{0}(\tau)-4\right)^{2}} \underset{\tau \rightarrow+\infty}{=} \frac{16 \hat{u}_{0}(\tau)}{\hat{r}_{0}(\tau)}\left(1+\mathcal{O}\left(\tau^{-\frac{2}{3}-\delta}\right)+\mathcal{O}\left(\tau^{\delta-2 \delta_{1}}\right)\right), \\
& \hat{r}_{0}(\tau)-4-\sqrt{32+\left(\hat{r}_{0}(\tau)-4\right)^{2}} \underset{\tau \rightarrow+\infty}{=}-\frac{2 \hat{r}_{0}(\tau)}{\hat{u}_{0}(\tau)}\left(1+\mathcal{O}\left(\tau^{-\frac{2}{3}-\delta}\right)+\mathcal{O}\left(\tau^{\delta-2 \delta_{1}}\right)\right) .
\end{aligned}
$$

Substituting the estimates (3.82) and (3.83) into Equation (3.71), one proves that

$$
\widehat{\mathcal{I}}_{\infty}(\tau) \underset{\tau \rightarrow+\infty}{=} \mathcal{I}_{\infty}^{\sharp}(\tau)+\mathcal{O}\left(\tau^{-\frac{2}{3}-\delta}\right)+\mathcal{O}\left(\tau^{\delta-2 \delta_{1}}\right),
$$

where $\mathcal{I}_{\infty}^{\sharp}(\tau)$ is defined by Equation (3.59), and, substituting the estimates (3.78)-(3.81) into Equation (3.76), one proves that

$$
\widehat{\mathcal{I}}_{0}(\tau) \underset{\tau \rightarrow+\infty}{=} \mathcal{I}_{0}^{\sharp}(\tau)+\mathcal{O}\left(\tau^{-\frac{2}{3}+\delta}\right)+\mathcal{O}\left(\tau^{-\frac{1}{3}+\delta-\delta_{1}}\right)+\mathcal{O}\left(\tau^{\delta-2 \delta_{1}}\right),
$$

where $\mathcal{I}_{0}^{\sharp}(\tau)$ is defined by Equation (3.61). Hence, via expansions (3.84) and (3.85), and Equations (3.69), (3.70), (3.74), and 3.75), one obtains the results stated in the Proposition.

Proposition 3.2.5. Let $T(\widetilde{\mu})$ be given in Equation (3.20), with $\mathcal{A}(\widetilde{\mu})$ defined by Equation (3.7), and $l(\widetilde{\mu})$ given in Equation (3.23) with the branches defined as in Corollary 3.2.4. Then

$$
\begin{aligned}
& T(\widetilde{\mu}) \underset{\substack{\tau \rightarrow+\infty \\
\operatorname{Re}(\widetilde{\mu}) \rightarrow+\infty}}{=}(b(\tau))^{-\frac{1}{2} \operatorname{ad}\left(\sigma_{3}\right)}\left(\mathrm{I}+\frac{1}{\widetilde{\mu}}\left(\begin{array}{cc}
0 & -2 \alpha^{4}\left(1+\hat{u}_{0}(\tau)\right) \\
\frac{\left(2-\hat{r}_{0}(\tau)\right) \alpha^{2}+\left(a-\frac{i}{2}\right) \tau^{-2 / 3}}{8 \alpha^{4}\left(1+\hat{u}_{0}(\tau)\right)} & 0
\end{array}\right)\right. \\
& \left.+\mathcal{O}\left(\frac{\left(c_{1}(\tau)\left(\hat{r}_{0}(\tau)\right)^{2}+c_{2}(\tau)\right)}{\widetilde{\mu}^{2}}\right)\right)
\end{aligned}
$$

and

$$
\begin{gathered}
T(\widetilde{\mu}) \underset{\substack{\tau \rightarrow+\infty \\
\operatorname{Re}(\widetilde{\mu}) \rightarrow+\infty}}{=} \frac{1}{\sqrt{2}}\left(\frac{b(\tau)}{2 \sqrt{2} \alpha^{3}}\right)^{-\frac{1}{2} \operatorname{ad}\left(\sigma_{3}\right)}\left(\left(\begin{array}{cc}
1 & 1 \\
-1 & 1
\end{array}\right)+\widetilde{\mu} \frac{\left(-2+\hat{r}_{0}(\tau)\right)}{4 \sqrt{2} \alpha}\left(\begin{array}{cc}
1 & -1 \\
1 & 1
\end{array}\right)\right. \\
\left.\quad+\mathcal{O}\left(\widetilde{\mu}^{2}\left(c_{3}(\tau)\left(\hat{r}_{0}(\tau)\right)^{2}+c_{4}(\tau) \hat{u}_{0}(\tau)+c_{5}(\tau)\right)\right)\right)
\end{gathered}
$$

where $c_{j}(\tau)={ }_{\tau \rightarrow+\infty} \mathcal{O}(1), j=1, \ldots, 5$.

Proof. Here, the proof of estimate (3.86) is presented: the estimate (3.87) is proven analogously. Via Equations (3.10), (3.11), and (3.14), and conditions (3.12), one shows that

$$
\begin{aligned}
& l(\widetilde{\mu}) \underset{\substack{\tau \rightarrow+\infty \\
\operatorname{Re}(\widetilde{\mu}) \rightarrow+\infty}}{=} 2 \widetilde{\mu}+\frac{1}{\widetilde{\mu}}\left(a-\frac{i}{2}\right) \tau^{-2 / 3}+\mathcal{O}\left(\widetilde{\mu}^{-3}\right), \\
& \mathrm{i}\left(\mathcal{A}(\widetilde{\mu})-\mathrm{i} l(\widetilde{\mu}) \sigma_{3}\right) \sigma_{3} \underset{\substack{\tau \rightarrow+\infty \\
\operatorname{Re}(\widetilde{\mu}) \rightarrow+\infty}}{=} 4 \mathrm{I} \widetilde{\mu}+\left(\begin{array}{cc}
0 & -\frac{4 \sqrt{-a(\tau) b(\tau)}}{b(\tau)} \\
-2 \mathrm{i} d(\tau) & 0
\end{array}\right)+\frac{1}{\widetilde{\mu}}\left(\left(-2+\hat{r}_{0}(\tau)\right) \alpha^{2}\right. \\
& \left.+\left(a-\frac{\mathrm{i}}{2}\right) \tau^{-2 / 3}\right) \mathrm{I}+\frac{1}{\widetilde{\mu}^{2}}\left(\begin{array}{cc}
0 & \frac{8 \alpha^{6}}{b(\tau)} \\
-b(\tau) & 0
\end{array}\right)+\mathcal{O}\left(\widetilde{\mu}^{-3} \mathrm{I}\right) \\
& \frac{1}{\sqrt{2 \mathrm{i} l(\widetilde{\mu})\left(\mathcal{A}_{11}(\widetilde{\mu})-\mathrm{i} l(\widetilde{\mu})\right)}} \underset{\substack{\tau \rightarrow(\vec{\mu}) \rightarrow+\infty \\
\operatorname{Re}()^{\prime}}}{=} \frac{1}{4 \widetilde{\mu}}\left(1-\frac{1}{8 \widetilde{\mu}^{2}}\left(\left(-2+\hat{r}_{0}(\tau)\right) \alpha^{2}+3\left(a-\frac{\mathrm{i}}{2}\right) \tau^{-2 / 3}\right)\right. \\
& \left.+\mathcal{O}\left(\frac{\left(C_{1}(\tau)\left(\hat{r}_{0}(\tau)\right)^{2}+C_{2}(\tau)\right)}{\widetilde{\mu}^{4}}\right)\right)
\end{aligned}
$$

where $C_{j}(\tau)={ }_{\tau \rightarrow+\infty} \mathcal{O}(1), j=1,2$; hence, via Equation (3.20) and conditions (3.12), one arrives at the estimate (3.86). 
Proposition 3.2.6. Let $T(\widetilde{\mu})$ be given in Equation (3.20), with $\mathcal{A}(\widetilde{\mu})$ defined by Equation (3.7), and $l^{2}(\widetilde{\mu})$ given in Equation (3.23). Under the conditions of Corollary 3.2.3.

$$
\begin{aligned}
& T_{11}(\widetilde{\mu})=T_{22}(\widetilde{\mu}) \underset{\substack{\widetilde{\mu}=\widetilde{\mu}_{0} \\
\tau \rightarrow+\infty}}{=} \frac{\alpha \hat{r}_{0}(\tau) \tau^{1 / 6}}{\sqrt{8 \sqrt{3} \alpha \hat{r}_{0}(\tau) \varpi \widetilde{\Lambda}}}\left(1-\frac{h_{0}(\tau)}{48 \alpha^{2} \widetilde{\Lambda}^{2}}-\frac{\left(a-\frac{i}{2}\right)}{48 \widetilde{\Lambda}^{2}}+\frac{1}{2 \alpha}\left(\frac{4-\hat{r}_{0}(\tau)+4 \sqrt{3} \varpi}{\hat{r}_{0}(\tau)}+\frac{7}{6}\right)\right. \\
& \left.\times \tau^{-1 / 3} \widetilde{\Lambda}+\mathcal{O}\left(\tau^{2 \delta-4 \varepsilon}\right)+\mathcal{O}\left(\tau^{-\varepsilon-\delta_{1}}\right)+\mathcal{O}\left(\tau^{2 \varepsilon-2 \delta_{1}}\right)\right), \\
& T_{12}(\widetilde{\mu}) \underset{\substack{\widetilde{\mu}=\widetilde{\mu}_{0} \\
\tau \rightarrow+\infty}}{=}-\frac{8 \alpha^{4} \hat{u}_{0}(\tau) \tau^{1 / 6}}{b(\tau) \sqrt{8 \sqrt{3} \alpha \hat{r}_{0}(\tau) \varpi \widetilde{\Lambda}}}\left(1-\frac{h_{0}(\tau)}{48 \alpha^{2} \widetilde{\Lambda}^{2}}-\frac{\left(a-\frac{\mathrm{i}}{2}\right)}{48 \widetilde{\Lambda}^{2}}-\left(\frac{1}{2 \alpha}\left(\frac{4-\hat{r}_{0}(\tau)+4 \sqrt{3} \varpi}{\hat{r}_{0}(\tau)}-\frac{7}{6}\right)\right.\right. \\
& \left.\left.-\frac{2}{\alpha \hat{u}_{0}(\tau)}\right) \tau^{-1 / 3} \widetilde{\Lambda}+\mathcal{O}\left(\tau^{2 \delta-4 \varepsilon}\right)+\mathcal{O}\left(\tau^{-\varepsilon-\delta_{1}}\right)\right), \\
& T_{21}(\widetilde{\mu}) \underset{\substack{\widetilde{\mu}=\widetilde{\mu}_{0} \\
\tau \rightarrow+\infty}}{=}-\frac{b(\tau) \tau^{1 / 6}}{2 \alpha^{2} \sqrt{8 \sqrt{3} \alpha \hat{r}_{0}(\tau) \varpi \widetilde{\Lambda}}}\left(\frac{\hat{r}_{0}(\tau)+2 \hat{u}_{0}(\tau)-\alpha^{-2}\left(a-\frac{\mathrm{i}}{2}\right) \tau^{-2 / 3}}{1+\hat{u}_{0}(\tau)}\right)\left(1-\frac{h_{0}(\tau)}{48 \alpha^{2} \widetilde{\Lambda}^{2}}-\frac{\left(a-\frac{\mathrm{i}}{2}\right)}{48 \widetilde{\Lambda}^{2}}\right. \\
& -\left(\frac{1}{2 \alpha}\left(\frac{4-\hat{r}_{0}(\tau)+4 \sqrt{3} \varpi}{\hat{r}_{0}(\tau)}-\frac{7}{6}\right)+\frac{4}{\alpha}\left(\frac{\hat{r}_{0}(\tau)+2 \hat{u}_{0}(\tau)-\alpha^{-2}\left(a-\frac{i}{2}\right) \tau^{-2 / 3}}{1+\hat{u}_{0}(\tau)}\right)^{-1}\right) \tau^{-1 / 3} \widetilde{\Lambda} \\
& \left.+\mathcal{O}\left(\tau^{2 \delta-4 \varepsilon}\right)+\mathcal{O}\left(\tau^{-\varepsilon-\delta_{1}}\right)\right),
\end{aligned}
$$

where

$$
\varpi:= \begin{cases}+1, & \arg (\widetilde{\Lambda})=0, \\ -1, & \arg (\widetilde{\Lambda})=\pi .\end{cases}
$$

Proof. Using the definition of $\mathcal{A}(\widetilde{\mu})$ (cf. Equation (3.7)), together with Equations (3.10), (3.11), and (3.14), and conditions (3.12) and those in Corollary 3.2.3, one derives the following expansions:

$$
\begin{aligned}
& \frac{1}{\sqrt{2 \mathrm{i} l(\widetilde{\mu})\left(\mathcal{A}_{11}(\widetilde{\mu})-\mathrm{i} l(\widetilde{\mu})\right)}} \underset{\tau \rightarrow \widetilde{\mu} \widetilde{\mu}_{0}}{=} \frac{\tau^{1 / 6}}{\sqrt{8 \sqrt{3} \alpha \hat{r}_{0}(\tau) \varpi \widetilde{\Lambda}}}\left(1-\frac{h_{0}(\tau)}{48 \alpha^{2} \widetilde{\Lambda}^{2}}-\frac{\left(a-\frac{\mathrm{i}}{2}\right)}{48 \widetilde{\Lambda}^{2}}-\frac{1}{2 \alpha}\left(\frac{4-\hat{r}_{0}(\tau)+4 \sqrt{3} \varpi}{\hat{r}_{0}(\tau)}\right.\right. \\
& \left.\left.-\frac{7}{6}\right) \tau^{-1 / 3} \widetilde{\Lambda}+\mathcal{O}\left(\frac{\left(h_{0}(\tau)\right)^{2}}{\widetilde{\Lambda}^{4}}\right)+\mathcal{O}\left(\frac{\tau^{-1 / 3} h_{0}(\tau)}{\hat{r}_{0}(\tau) \widetilde{\Lambda}}\right)\right), \\
& \mathrm{i} \mathcal{A}_{11}(\widetilde{\mu})+l(\widetilde{\mu}) \underset{\substack{\widetilde{\mu}=\widetilde{\mu}_{0} \\
\tau \rightarrow+\infty}}{=} \alpha \hat{r}_{0}(\tau)\left(1+\left(\frac{4-\hat{r}_{0}(\tau)+4 \sqrt{3} \varpi}{\alpha \hat{r}_{0}(\tau)}+\frac{4 \sqrt{3} \varpi}{\alpha \hat{r}_{0}(\tau)}\left(\frac{h_{0}(\tau)}{24 \alpha^{2} \widetilde{\Lambda}^{2}}+\frac{\left(a-\frac{\mathrm{i}}{2}\right)}{24 \widetilde{\Lambda}^{2}}\right)\right) \tau^{-1 / 3} \widetilde{\Lambda}\right. \\
& \left.+\left(\frac{-2+\hat{r}_{0}(\tau)-\frac{14}{\sqrt{3}} \varpi}{\alpha^{2} \hat{r}_{0}(\tau)}\right) \tau^{-2 / 3} \widetilde{\Lambda}^{2}+\mathcal{O}\left(\frac{\tau^{-1 / 3}\left(h_{0}(\tau)\right)^{2}}{\hat{r}_{0}(\tau) \widetilde{\Lambda}^{3}}\right)\right), \\
& -\mathrm{i} \mathcal{A}_{12}(\widetilde{\mu}) \underset{\substack{\widetilde{\mu}=\widetilde{\mu}_{0} \\
\tau \rightarrow+\infty}}{=} \frac{1}{b(\tau)}\left(-8 \alpha^{4} \hat{u}_{0}(\tau)-16 \alpha^{3} \tau^{-1 / 3} \widetilde{\Lambda}+24 \alpha^{2} \tau^{-2 / 3} \widetilde{\Lambda}^{2}+\mathcal{O}\left(\tau^{-1} \widetilde{\Lambda}^{3}\right)\right), \\
& \mathrm{i} \mathcal{A}_{21}(\widetilde{\mu}) \underset{\substack{\tilde{\mu}=\widetilde{\mu}_{0} \\
\tau \rightarrow+\infty}}{=} b(\tau)\left(-\frac{\left(-2+\hat{r}_{0}(\tau)\right)}{2 \alpha^{2}\left(1+\hat{u}_{0}(\tau)\right)}-\frac{1}{\alpha^{2}}+\frac{\left(a-\frac{i}{2}\right) \tau^{-2 / 3}}{2 \alpha^{4}\left(1+\hat{u}_{0}(\tau)\right)}+\frac{2}{\alpha^{3}} \tau^{-1 / 3} \widetilde{\Lambda}-\frac{3}{\alpha^{4}} \tau^{-2 / 3} \widetilde{\Lambda}^{2}\right. \\
& \left.+\mathcal{O}\left(\tau^{-1} \widetilde{\Lambda}^{3}\right)\right) .
\end{aligned}
$$

According to the choice of the branch of $l(\xi)$ in Corollary 3.2.4 $l(\xi)>0$ for positive $\xi$ outside the neighborhood of $\xi=\alpha$ containing the double-turning points; therefore, one arrives at the definition of $\varpi$ stated in the Proposition. The asymptotics (3.88)-(3.90) are obtained by substituting the above expansions into Equations (3.22). 


\subsection{The Model Problem and Asymptotics Near the Turning Points}

For the calculation of the monodromy data, one needs an approximation that is more accurate than that given by the WKB formula (cf. Equation (3.18)) for the solution of Equation (3.6) in proper neighborhoods of the turning points. There are two simple turning points approaching $\pm \mathrm{i} \sqrt{2} \alpha$ : the approximate solution of Equation (3.6) in neighborhoods of these turning points is given in terms of Airy functions. There are also two pairs of turning points, one pair coalescing at $-\alpha$ and another pair coalescing at $+\alpha$ (double-turning points): it is well known that, in neighborhoods of $\pm \alpha$, the approximate solution of Equation (3.6) is expressed in terms of parabolic-cylinder functions (see, for example, [8, 9]). In order to obtain asymptotics of $u(\tau)$, and the associated functions $\mathcal{H}(\tau)$ and $f(\tau)$, it is sufficient to study a subset of the complete set of the monodromy data, which can be calculated via the approximation of the general solution of Equation (3.6) in a neighborhood of the doubleturning point $+\alpha$. For the asymptotic conditions (3.12) on the functions $\hat{r}_{0}(\tau), \hat{u}_{0}(\tau)$, and $h_{0}(\tau)$, this approximation is not straightforward, and is given in Lemma 3.3 .1 below.

Lemma 3.3.1. Let $\widetilde{\mu}=\alpha+\tau^{-1 / 3} \widetilde{\Lambda}$, where $\widetilde{\Lambda}={ }_{\tau \rightarrow+\infty} \mathcal{O}\left(\tau^{\varepsilon}\right), 0<\varepsilon<1 / 9$, and

$$
\nu+1:=-\frac{\mathrm{i}}{2 \sqrt{3}}\left(\frac{h_{0}(\tau)}{\alpha^{2}}+\left(a-\frac{\mathrm{i}}{2}\right)-\frac{\mathfrak{p}_{\tau}}{4}\right)+\frac{1}{2},
$$

where $\mathfrak{p}_{\tau}$ is defined by Equation (3.35). In conjunction with Equations (3.9)-(3.11) and (3.13), and conditions (3.12), impose the following restrictions:

$$
\begin{gathered}
0 \underset{\tau \rightarrow+\infty}{<} \operatorname{Re}(\nu+1) \underset{\tau \rightarrow+\infty}{<} 1, \quad \operatorname{Im}(\nu+1) \underset{\tau \rightarrow+\infty}{=} \mathcal{O}(1), \\
0<\delta<\varepsilon<\frac{1}{9}, \quad 6 \varepsilon+2 \varepsilon \operatorname{Re}(\nu+1)+\frac{\delta}{2}<\delta_{1}<\frac{1}{3} .
\end{gathered}
$$

Then there exists a fundamental solution of Equation (3.6) with asymptotic representation

$$
\widetilde{\Psi}(\widetilde{\mu}, \tau) \underset{\tau \rightarrow+\infty}{=} \mathcal{F}_{\tau}(\widetilde{\Lambda})\left(\mathrm{I}+\mathcal{O}\left(\tau^{6 \varepsilon+2 \varepsilon \operatorname{Re}(\nu+1)+\frac{\delta}{2}-\delta_{1}}\right)\right) \psi_{0}(\widetilde{\Lambda})
$$

where

$$
\mathcal{F}_{\tau}(\widetilde{\Lambda}):=\left(\begin{array}{cc}
\frac{\mathrm{i} \tau^{1 / 6} \sqrt{-8 \alpha^{4} \hat{u}_{0}(\tau)}}{\sqrt{b(\tau)} \sqrt{\varkappa_{0}(\tau)}} & 0 \\
\frac{\mathrm{i} \sqrt{b(\tau)}\left(\alpha \hat{r}_{0}(\tau) \tau^{1 / 6}-\mathrm{i} \tau^{-1 / 6} \ell_{\tau} \widetilde{\Lambda}\right)}{\sqrt{\varkappa_{0}(\tau)} \sqrt{-8 \alpha^{4} \hat{u}_{0}(\tau)}} & -\frac{\mathrm{i} \sqrt{\varkappa_{0}(\tau)} \sqrt{b(\tau)} \tau^{-1 / 6}}{\sqrt{-8 \alpha^{4} \hat{u}_{0}(\tau)}}
\end{array}\right),
$$

$\varkappa_{0}^{2}(\tau)$ is defined by Equation (3.65),

$$
\ell_{\tau}=-\mathfrak{p}_{\tau}+\sqrt{\mathfrak{p}_{\tau}^{2}-\hat{r}_{0}(\tau)\left(8-\hat{r}_{0}(\tau)\right)} \underset{\tau \rightarrow+\infty}{=} \ell_{\tau}^{\infty}+\mathcal{O}\left(\frac{\tau^{-2 / 3} \varkappa_{0}^{2}(\tau)\left(\mathcal{O}(1)+\left(\hat{r}_{0}(\tau)\right)^{2}\right)}{\left(\hat{r}_{0}(\tau)\right)^{2}}\right),
$$

with

$$
\ell_{\tau}^{\infty}:=-\mathfrak{p}_{\tau}+\mathrm{i} 4 \sqrt{3}
$$

and $\psi_{0}(\widetilde{\Lambda})$ is a fundamental solution of

$$
\frac{\partial \psi_{0}(\widetilde{\Lambda})}{\partial \widetilde{\Lambda}}=\left(\mathrm{i} 4 \sqrt{3} \widetilde{\Lambda} \sigma_{3}+\widetilde{q}(\tau) \sigma_{-}+\widetilde{p}(\tau) \sigma_{+}\right) \psi_{0}(\widetilde{\Lambda})
$$

where

$$
\widetilde{p}(\tau)=-\mathrm{i} \varkappa_{0}(\tau), \quad \widetilde{q}(\tau)=-\frac{\mathrm{i}}{\varkappa_{0}(\tau)}\left(\varkappa_{0}^{2}(\tau)+\ell_{\tau}^{\infty}+\frac{4\left(a-\frac{\mathrm{i}}{2}\right) \hat{u}_{0}(\tau)}{1+\hat{u}_{0}(\tau)}\right) .
$$

The function $\psi_{0}(\widetilde{\Lambda})$ can be explicitly presented in the following form:

$$
\psi_{0}(\widetilde{\Lambda})=\left(\begin{array}{cc}
D_{-1-\nu}\left(\mathrm{ie}^{\mathrm{i} \frac{\mathrm{i} \pi}{4}} 2^{3 / 2} 3^{1 / 4} \widetilde{\Lambda}\right) & D_{\nu}\left(\mathrm{e}^{\mathrm{i} \frac{\mathrm{i}}{4}} 2^{3 / 2} 3^{1 / 4} \widetilde{\Lambda}\right) \\
\hat{\partial}_{\widetilde{\Lambda}} D_{-1-\nu}\left(\mathrm{ie}^{\frac{\mathrm{i} \pi}{4}} 2^{3 / 2} 3^{1 / 4} \widetilde{\Lambda}\right) & \hat{\partial}_{\widetilde{\Lambda}} D_{\nu}\left(\mathrm{e}^{\frac{\mathrm{i} \pi}{4}} 2^{3 / 2} 3^{1 / 4} \widetilde{\Lambda}\right)
\end{array}\right),
$$

where $D_{*}(\cdot)$ is the parabolic-cylinder function [11], and $\hat{\partial}_{\widetilde{\Lambda}}:=(\widetilde{p}(\tau))^{-1}\left(\frac{\partial}{\partial \Lambda}-\mathrm{i} 4 \sqrt{3} \widetilde{\Lambda}\right)$. 
Proof. The derivation of approximation (3.94) consists of the following sequence of invertible linear transformations, $F_{j}: \mathrm{SL}_{2}(\mathbb{C}) \rightarrow \mathrm{SL}_{2}(\mathbb{C}), j=1, \ldots, 7$ :

$$
\begin{aligned}
\text { (i) } \quad F_{1}: \widetilde{\Psi}(\widetilde{\mu}) \mapsto \Phi(\widetilde{\Lambda}):=\widetilde{\Psi}\left(\alpha+\tau^{-1 / 3} \widetilde{\Lambda}\right), \\
\text { (ii) } \quad F_{2}: \Phi(\widetilde{\Lambda}) \mapsto \widehat{\Phi}(\widetilde{\Lambda}):=(b(\tau))^{\frac{1}{2} \sigma_{3}} \Phi(\widetilde{\Lambda}), \\
\text { (iii) } \quad F_{3}: \widehat{\Phi}(\widetilde{\Lambda}) \mapsto \phi(\widetilde{\Lambda}):=(\mathcal{N}(\tau))^{-1} \widehat{\Phi}(\widetilde{\Lambda}), \\
\text { (iv) } \quad F_{4}: \phi(\widetilde{\Lambda}) \mapsto \widetilde{\Phi}(\widetilde{\Lambda}):=\tau^{-\frac{1}{6} \sigma_{3}} \phi(\widetilde{\Lambda}), \\
\text { (v) } \quad F_{5}: \widetilde{\Phi}(\widetilde{\Lambda}) \mapsto \widehat{\phi}(\widetilde{\Lambda}):=\left(\begin{array}{c}
1 \\
-\ell_{\tau} \widetilde{\Lambda} 1
\end{array}\right) \widetilde{\Phi}(\widetilde{\Lambda}), \\
\text { (vi) } \quad F_{6}: \widehat{\phi}(\widetilde{\Lambda}) \mapsto \psi(\widetilde{\Lambda}):=(\mathcal{G}(\tau))^{-1} \widehat{\phi}(\widetilde{\Lambda}), \\
\text { (vii) } \quad F_{7}: \psi(\widetilde{\Lambda}) \mapsto \psi_{0}(\widetilde{\Lambda}):=\left(\chi_{0}(\widetilde{\Lambda})\right)^{-1} \psi(\widetilde{\Lambda}),
\end{aligned}
$$

where the unimodular, matrix-valued functions $\mathcal{N}(\tau), \mathcal{G}(\tau)$, and $\chi_{0}(\widetilde{\Lambda})$, respectively, are described in steps (iii), (vi), and (vii) below.

(i) Let $\widetilde{\Psi}(\widetilde{\mu})$ solve Equation (3.6). Then applying the transformation $F_{1}$, using Equations (3.10), (3.11), and (3.14), and conditions (3.12), one shows that

$$
\frac{\partial \Phi(\widetilde{\Lambda})}{\partial \widetilde{\Lambda}} \underset{\tau \rightarrow+\infty}{=}\left(\left(\tau^{1 / 3} \mathcal{Q}_{+}+\tau^{-1 / 3} \mathcal{Q}_{-}\right)+\widetilde{\Lambda} \mathcal{Q}_{1}+\tau^{-1 / 3} \widetilde{\Lambda}^{2} \mathcal{Q}_{2}+\mathcal{O}\left(\tau^{-2 / 3} \widetilde{\Lambda}^{3} \mathcal{Q}_{3}\right)\right) \Phi(\widetilde{\Lambda}),
$$

where

$$
\mathcal{Q}_{ \pm}=(b(\tau))^{-\frac{1}{2} \operatorname{ad}\left(\sigma_{3}\right)} \hat{\mathcal{P}}_{ \pm}, \quad \mathcal{Q}_{j}=(b(\tau))^{-\frac{1}{2} \operatorname{ad}\left(\sigma_{3}\right)} \hat{\mathcal{P}}_{j}, \quad j=1,2,3
$$

with

$$
\begin{array}{cc}
\hat{\mathcal{P}}_{+}:=\left(\begin{array}{cc}
-\mathrm{i} \alpha \hat{r}_{0}(\tau) & -8 \mathrm{i} \alpha^{4} \hat{u}_{0}(\tau) \\
\frac{\mathrm{i}\left(\hat{r}_{0}(\tau)+2 \hat{u}_{0}(\tau)\right)}{2 \alpha^{2}\left(1+\hat{u}_{0}(\tau)\right)} & \mathrm{i} \alpha \hat{r}_{0}(\tau)
\end{array}\right), & \hat{\mathcal{P}}_{-}:=\left(\begin{array}{cc}
0 & 0 \\
-\frac{\mathrm{i}\left(a-\frac{\mathrm{i}}{2}\right)}{2 \alpha^{4}\left(1+\hat{u}_{0}(\tau)\right)} & 0
\end{array}\right) \\
\hat{\mathcal{P}}_{1}:=\left(\begin{array}{ccc}
\mathrm{i}\left(-4+\hat{r}_{0}(\tau)\right) & -16 \mathrm{i} \alpha^{3} \\
-\frac{2 \mathrm{i}}{\alpha^{3}} & -\mathrm{i}\left(-4+\hat{r}_{0}(\tau)\right)
\end{array}\right), & \hat{\mathcal{P}}_{2}:=\left(\begin{array}{cc}
-\frac{\mathrm{i}\left(-2+\hat{r}_{0}(\tau)\right)}{\alpha} & 24 \mathrm{i} \alpha^{2} \\
\frac{3 \mathrm{i}}{\alpha^{4}} & \frac{\mathrm{i}\left(-2+\hat{r}_{0}(\tau)\right)}{\alpha}
\end{array}\right) \\
\hat{\mathcal{P}}_{3}:=\left(\begin{array}{cc}
\mathcal{O}(1)+\mathcal{O}\left(\hat{r}_{0}(\tau)\right) & \mathcal{O}(1) \\
\mathcal{O}(1) & \mathcal{O}(1)+\mathcal{O}\left(\hat{r}_{0}(\tau)\right)
\end{array}\right)
\end{array}
$$

Note that $\operatorname{tr}\left(\mathcal{Q}_{ \pm}\right)=\operatorname{tr}\left(\hat{\mathcal{P}}_{ \pm}\right)=\operatorname{tr}\left(\mathcal{Q}_{j}\right)=\operatorname{tr}\left(\hat{\mathcal{P}}_{j}\right)=0, j=1,2,3$; furthermore, Equation (3.77) implies

$$
\operatorname{det}\left(\hat{\mathcal{P}}_{+}\right)=-8 \alpha^{2}\left(\frac{\left(\hat{u}_{0}(\tau)\right)^{2}}{1+\hat{u}_{0}(\tau)}+\frac{\hat{r}_{0}(\tau) \hat{u}_{0}(\tau)}{2\left(1+\hat{u}_{0}(\tau)\right)}-\frac{\left(\hat{r}_{0}(\tau)\right)^{2}}{8}\right)=\varkappa_{0}^{2}(\tau) \tau^{-2 / 3},
$$

where $\varkappa_{0}^{2}(\tau)$ is defined by Equation (3.65).

(ii) Let $\Phi(\widetilde{\Lambda})$ solve Equation (3.101). Then applying the transformation $F_{2}$, one obtains

$$
\frac{\partial \widehat{\Phi}(\widetilde{\Lambda})}{\partial \widetilde{\Lambda}} \underset{\tau \rightarrow+\infty}{=}\left(\left(\tau^{1 / 3} \hat{\mathcal{P}}_{+}+\tau^{-1 / 3} \hat{\mathcal{P}}_{-}\right)+\widetilde{\Lambda} \hat{\mathcal{P}}_{1}+\tau^{-1 / 3} \widetilde{\Lambda}^{2} \hat{\mathcal{P}}_{2}+\mathcal{O}\left(\tau^{-2 / 3} \widetilde{\Lambda}^{3} \hat{\mathcal{P}}_{3}\right)\right) \widehat{\Phi}(\widetilde{\Lambda}) .
$$

(iii) The idea behind the following transformation for Equation (3.103) is to put the matrix $\hat{\mathcal{P}}_{+}$ into Jordan canonical form, that is, to find a function $\mathcal{N}(\tau)$ such that

$$
(\mathcal{N}(\tau))^{-1} \hat{\mathcal{P}}_{+} \mathcal{N}(\tau)=\mathrm{i} \varkappa_{0}(\tau) \tau^{-1 / 3} \sigma_{3}+\sigma_{+} .
$$

The following solution for $\mathcal{N}(\tau)$ is chosen:

$$
\mathcal{N}(\tau)=\left(\begin{array}{cc}
\sqrt{-8 \mathrm{i} \alpha^{4} \hat{u}_{0}(\tau)} & 0 \\
\frac{\mathrm{i}\left(\alpha \hat{r}_{0}(\tau)+\varkappa_{0}(\tau) \tau^{-1 / 3}\right)}{\sqrt{-8 \mathrm{i} \alpha^{4} \hat{u}_{0}(\tau)}} & \frac{1}{\sqrt{-8 \mathrm{i} \alpha^{4} \hat{u}_{0}(\tau)}}
\end{array}\right) .
$$


Let $\widehat{\Phi}(\widetilde{\Lambda})$ solve Equation (3.103). Then applying the transformation $F_{3}$, using Equations (3.10) and (3.11), conditions (3.12), and Equations (3.77), (3.65), and (3.102), one shows that

$$
\frac{\partial \phi(\widetilde{\Lambda})}{\partial \widetilde{\Lambda}} \underset{\tau \rightarrow+\infty}{=}\left(\left(\tau^{1 / 3} \mathcal{P}_{+}^{\sharp}+\tau^{-1 / 3} \mathcal{P}_{-}^{\sharp}\right)+\widetilde{\Lambda} \mathcal{P}_{1}^{\sharp}+\tau^{-1 / 3} \widetilde{\Lambda}^{2} \mathcal{P}_{2}^{\sharp}+\mathcal{O}\left(\tau^{-2 / 3} \widetilde{\Lambda}^{3}(\mathcal{N}(\tau))^{-1} \hat{\mathcal{P}}_{3} \mathcal{N}(\tau)\right)\right) \phi(\widetilde{\Lambda}),
$$

where $\mathcal{P}_{ \pm}^{\sharp}:=(\mathcal{N}(\tau))^{-1} \mathcal{P}_{ \pm} \mathcal{N}(\tau), \mathcal{P}_{j}^{\sharp}:=(\mathcal{N}(\tau))^{-1} \hat{\mathcal{P}}_{j} \mathcal{N}(\tau), j=1,2$, with

$$
\begin{aligned}
& \mathcal{P}_{+}^{\sharp}=\mathrm{i} \varkappa_{0}(\tau) \tau^{-1 / 3} \sigma_{3}+\sigma_{+}, \quad \mathcal{P}_{-}^{\sharp}=-\frac{4\left(a-\frac{\mathrm{i}}{2}\right) \hat{u}_{0}(\tau)}{\left(1+\hat{u}_{0}(\tau)\right)} \sigma_{-}, \\
& \left(\mathcal{P}_{1}^{\sharp}\right)_{11}=-\left(\mathcal{P}_{1}^{\sharp}\right)_{22} \underset{\tau \rightarrow+\infty}{=} \mathfrak{p}_{\tau}+\frac{2 \mathrm{i} \varkappa_{0}(\tau) \tau^{-1 / 3}}{\alpha \hat{u}_{0}(\tau)}+\mathcal{O}\left(\frac{\varkappa_{0}^{2}(\tau) \tau^{-2 / 3}}{\hat{r}_{0}(\tau) \hat{u}_{0}(\tau)}\right)+\mathcal{O}\left(\frac{\varkappa_{0}^{2}(\tau) \tau^{-2 / 3}}{\hat{r}_{0}(\tau)}\right), \\
& \left(\mathcal{P}_{1}^{\sharp}\right)_{12}=\frac{2}{\alpha \hat{u}_{0}(\tau)}, \quad\left(\mathcal{P}_{1}^{\sharp}\right)_{21} \underset{\tau \rightarrow+\infty}{=}-2 \mathrm{i} \varkappa_{0}(\tau) \mathfrak{p}_{\tau} \tau^{-1 / 3}+\mathcal{O}\left(\frac{\varkappa_{0}^{2}(\tau) \tau^{-2 / 3}}{\hat{u}_{0}(\tau)}\right)+\mathcal{O}\left(\varkappa_{0}^{2}(\tau) \tau^{-2 / 3}\right), \\
& \left(\mathcal{P}_{2}^{\sharp}\right)_{11}=-\left(\mathcal{P}_{2}^{\sharp}\right)_{22}=-\frac{\mathrm{i}\left(6+\left(-2+\hat{r}_{0}(\tau)\right)\left(3+\hat{u}_{0}(\tau)\right)\right)}{\alpha \hat{u}_{0}(\tau)}-\frac{3 \mathrm{i} \varkappa_{0}(\tau) \tau^{-1 / 3}}{\alpha^{2} \hat{u}_{0}(\tau)}, \quad\left(\mathcal{P}_{2}^{\sharp}\right)_{12}=-\frac{3}{\alpha^{2} \hat{u}_{0}(\tau)}, \\
& \left(\mathcal{P}_{2}^{\sharp}\right)_{21} \underset{\tau \rightarrow+\infty}{=}-\hat{r}_{0}(\tau)\left(8-\hat{r}_{0}(\tau)\right)-\frac{2 \varkappa_{0}(\tau)}{\alpha \hat{u}_{0}(\tau)}\left(6+\left(-2+\hat{r}_{0}(\tau)\right)\left(3+\hat{u}_{0}(\tau)\right)\right) \tau^{-1 / 3} \\
& +\mathcal{O}\left(\frac{\varkappa_{0}^{2}(\tau) \tau^{-2 / 3}}{\hat{u}_{0}(\tau)}\right)+\mathcal{O}\left(\varkappa_{0}^{2}(\tau) \tau^{-2 / 3}\right),
\end{aligned}
$$

where $\mathfrak{p}_{\tau}$ is defined by Equation (3.35).

(iv) Let $\phi(\widetilde{\Lambda})$ solve Equation (3.104). Then applying the transformation $F_{4}$, one proves that

$$
\begin{aligned}
\frac{\partial \widetilde{\Phi}(\widetilde{\Lambda})}{\partial \widetilde{\Lambda}} \underset{\tau \rightarrow+\infty}{=}\left(\left(\begin{array}{cc}
\mathrm{i} \varkappa_{0}(\tau) & 1 \\
-\frac{4\left(a-\frac{i}{2}\right) \hat{u}_{0}(\tau)}{1+\hat{u}_{0}(\tau)} & -\mathrm{i} \varkappa_{0}(\tau)
\end{array}\right)+\widetilde{\Lambda}\left(\begin{array}{cc}
\mathfrak{p}_{\tau} & 0 \\
-2 \mathrm{i} \varkappa_{0}(\tau) \mathfrak{p}_{\tau} & -\mathfrak{p}_{\tau}
\end{array}\right)\right. \\
\left.+\widetilde{\Lambda}^{2}\left(\begin{array}{cc}
0 & 0 \\
-\hat{r}_{0}(\tau)\left(8-\hat{r}_{0}(\tau)\right) & 0
\end{array}\right)+\mathcal{O}\left(\tau^{-1 / 3} E_{\tau}(\widetilde{\Lambda})\right)\right) \widetilde{\Phi}(\widetilde{\Lambda}),
\end{aligned}
$$

where, with the help of conditions (3.12),

$$
\begin{aligned}
& E_{\tau}(\widetilde{\Lambda}) \underset{\tau \rightarrow+\infty}{=} \tilde{\Lambda}\left(\begin{array}{cc}
\mathcal{O}\left(\frac{\varkappa_{0}(\tau)}{\hat{u}_{0}(\tau)}\right)+\mathcal{O}\left(\frac{\varkappa_{0}^{2}(\tau) \tau^{-1 / 3}}{\hat{r}_{0}(\tau) \hat{u}_{0}(\tau)}\right) & \mathcal{O}\left(\left(\hat{u}_{0}(\tau)\right)^{-1}\right) \\
\mathcal{O}\left(\frac{\varkappa_{0}^{2}(\tau)}{\hat{u}_{0}(\tau)}\right)+\mathcal{O}\left(\varkappa_{0}^{2}(\tau)\right) & \mathcal{O}\left(\frac{\varkappa_{0}(\tau)}{\hat{u}_{0}(\tau)}\right)+\mathcal{O}\left(\frac{\varkappa_{0}^{2}(\tau) \tau^{-1 / 3}}{\hat{r}_{0}(\tau) \hat{u}_{0}(\tau)}\right)
\end{array}\right) \\
& +\widetilde{\Lambda}^{2}\left(\begin{array}{cc}
\mathcal{O}(1)+\mathcal{O}\left(\hat{r}_{0}(\tau)\right) & \mathcal{O}\left(\tau^{-1 / 3}\left(\hat{u}_{0}(\tau)\right)^{-1}\right) \\
\mathcal{O}\left(\varkappa_{0}(\tau)\right)+\mathcal{O}\left(\varkappa_{0}(\tau) \hat{r}_{0}(\tau)\right) & \mathcal{O}(1)+\mathcal{O}\left(\hat{r}_{0}(\tau)\right)
\end{array}\right) \\
& +\widetilde{\Lambda}^{3}\left(\begin{array}{cc}
\mathcal{O}\left(\tau^{-1 / 3}\right)+\mathcal{O}\left(\tau^{-1 / 3} \hat{r}_{0}(\tau)\right) & \mathcal{O}\left(\tau^{-2 / 3}\left(\hat{u}_{0}(\tau)\right)^{-1}\right) \\
\mathcal{O}\left(\hat{r}_{0}(\tau)\right)+\mathcal{O}\left(\left(\hat{r}_{0}(\tau)\right)^{2}\right) & \mathcal{O}\left(\tau^{-1 / 3}\right)+\mathcal{O}\left(\tau^{-1 / 3} \hat{r}_{0}(\tau)\right)
\end{array}\right) \\
& \left.=\begin{array}{cc}
\mathcal{O}\left(\frac{\tilde{\Lambda} \varkappa_{0}^{2}(\tau) \tau^{-1 / 3}}{\hat{r}_{0}(\tau) \hat{u}_{0}(\tau)}\right)+\mathcal{O}\left(\widetilde{\Lambda}^{2}\right)+\mathcal{O}\left(\widetilde{\Lambda}^{2} \hat{r}_{0}(\tau)\right) & \mathcal{O}\left(\widetilde{\Lambda}\left(\hat{u}_{0}(\tau)\right)^{-1}\right) \\
\mathcal{O}\left(\frac{\widetilde{\Lambda} \varkappa_{0}^{2}(\tau)}{\hat{u}_{0}(\tau)}\right)+\mathcal{O}\left(\widetilde{\Lambda}^{2} \varkappa_{0}(\tau)\right)+\mathcal{O}\left(\widetilde{\Lambda}^{3}\left(\hat{r}_{0}(\tau)\right)^{2}\right) & \mathcal{O}\left(\frac{\widetilde{\Lambda}_{0}^{2}(\tau) \tau^{-1 / 3}}{\hat{r}_{0}(\tau) \hat{u}_{0}(\tau)}\right)+\mathcal{O}\left(\widetilde{\Lambda}^{2}\right)+\mathcal{O}\left(\widetilde{\Lambda}^{2} \hat{r}_{0}(\tau)\right)
\end{array}\right)
\end{aligned}
$$

(v) One now proceeds to eliminate the $\widetilde{\Lambda}^{2}$ term in Equation (3.105). Applying the transformation $F_{5}$, with a $\tau$-dependent parameter $\ell_{\tau}$, one obtains

$$
\begin{aligned}
\frac{\partial \widehat{\phi}(\widetilde{\Lambda})}{\partial \widetilde{\Lambda}} \underset{\tau \rightarrow+\infty}{=}\left(\left(\begin{array}{cc}
\mathrm{i} \varkappa_{0}(\tau) & 1 \\
-\ell_{\tau}-\frac{4\left(a-\frac{i}{2}\right) \hat{u}_{0}(\tau)}{1+\hat{u}_{0}(\tau)} & -\mathrm{i} \varkappa_{0}(\tau)
\end{array}\right)+\widetilde{\Lambda}\left(\begin{array}{cc}
\mathfrak{p}_{\tau}+\ell_{\tau} & 0 \\
-2 \mathrm{i} \varkappa_{0}(\tau)\left(\ell_{\tau}+\mathfrak{p}_{\tau}\right) & -\left(\mathfrak{p}_{\tau}+\ell_{\tau}\right)
\end{array}\right)\right. \\
\left.+\widetilde{\Lambda}^{2}\left(\begin{array}{ccc}
0 & 0 \\
-\hat{r}_{0}(\tau)\left(8-\hat{r}_{0}(\tau)\right)-2 \mathfrak{p}_{\tau} \ell_{\tau}-\ell_{\tau}^{2} & 0
\end{array}\right)+\mathcal{O}\left(\tau^{-1 / 3} \widehat{E}_{\tau}(\widetilde{\Lambda})\right)\right) \widehat{\phi}(\widetilde{\Lambda})
\end{aligned}
$$

where

$$
\widehat{E}_{\tau}(\widetilde{\Lambda}):=\left(\begin{array}{cc}
1 & 0 \\
-\ell_{\tau} \widetilde{\Lambda} & 1
\end{array}\right) E_{\tau}(\widetilde{\Lambda})\left(\begin{array}{cc}
1 & 0 \\
\ell_{\tau} \widetilde{\Lambda} & 1
\end{array}\right)
$$


One now chooses $\ell_{\tau}$ so that the quadratic term in Equation (3.107) is annihilated:

$$
\ell_{\tau}^{2}+2 \mathfrak{p}_{\tau} \ell_{\tau}+\hat{r}_{0}(\tau)\left(8-\hat{r}_{0}(\tau)\right)=0
$$

thus,

$$
\ell_{\tau}=-\mathfrak{p}_{\tau}+\sqrt{\mathfrak{p}_{\tau}^{2}-\hat{r}_{0}(\tau)\left(8-\hat{r}_{0}(\tau)\right)} .
$$

A straightforward calculation shows that (cf. Equation (3.68)

$$
\mathfrak{p}_{\tau}^{2}-\hat{r}_{0}(\tau)\left(8-\hat{r}_{0}(\tau)\right) \underset{\tau \rightarrow+\infty}{=}-48+\mathcal{O}\left(\frac{\tau^{-2 / 3} \varkappa_{0}^{2}(\tau)\left(\mathcal{O}(1)+\left(\hat{r}_{0}(\tau)\right)^{2}\right)}{\left(\hat{r}_{0}(\tau)\right)^{2}}\right)
$$

hence, making the choice $\sqrt{-1}=\mathrm{i}$,

$$
\ell_{\tau} \underset{\tau \rightarrow+\infty}{=} \ell_{\tau}^{\infty}+\mathcal{O}\left(\frac{\tau^{-2 / 3} \varkappa_{0}^{2}(\tau)\left(\mathcal{O}(1)+\left(\hat{r}_{0}(\tau)\right)^{2}\right)}{\left(\hat{r}_{0}(\tau)\right)^{2}}\right)
$$

where $\ell_{\tau}^{\infty}$ is defined by Equation (3.97). Now, with the help of Equations (3.106), (3.108), and (3.110), and conditions (3.12), one re-writes Equation (3.107) as

$$
\frac{\partial \widehat{\phi}(\widetilde{\Lambda})}{\partial \widehat{\Lambda}} \underset{\tau \rightarrow+\infty}{=}\left(\left(\begin{array}{cc}
\mathrm{i} \varkappa_{0}(\tau) & 1 \\
-\ell_{\tau}^{\infty}-\frac{4\left(a-\frac{\mathrm{i}}{2}\right) \hat{u}_{0}(\tau)}{1+\hat{u}_{0}(\tau)} & -\mathrm{i} \varkappa_{0}(\tau)
\end{array}\right)+\widetilde{\Lambda}\left(\begin{array}{cc}
\mathrm{i} 4 \sqrt{3} & 0 \\
8 \sqrt{3} \varkappa_{0}(\tau) & -\mathrm{i} 4 \sqrt{3}
\end{array}\right)+\mathcal{O}\left(\widetilde{E}_{\tau}(\widetilde{\Lambda})\right)\right) \widehat{\phi}(\widetilde{\Lambda}),
$$

where

$$
\widetilde{E}_{\tau}(\widetilde{\Lambda}):=\left(\begin{array}{ll}
\left(\widetilde{E}_{\tau}(\widetilde{\Lambda})\right)_{11} & \left(\widetilde{E}_{\tau}(\widetilde{\Lambda})\right)_{12} \\
\left(\widetilde{E}_{\tau}(\widetilde{\Lambda})\right)_{21} & \left(\widetilde{E}_{\tau}(\widetilde{\Lambda})\right)_{22}
\end{array}\right)
$$

with

$$
\begin{aligned}
& \left(\widetilde{E}_{\tau}(\widetilde{\Lambda})\right)_{11}=-\left(\widetilde{E}_{\tau}(\widetilde{\Lambda})\right)_{22} \underset{\tau \rightarrow+\infty}{=} \mathcal{O}\left(\frac{\widetilde{\Lambda} \tau^{-2 / 3} \varkappa_{0}^{2}(\tau)}{\left(\hat{r}_{0}(\tau)\right)^{2}}\right)+\mathcal{O}\left(\widetilde{\Lambda} \tau^{-2 / 3} \varkappa_{0}^{2}(\tau)\right) \\
& +\mathcal{O}\left(\widetilde{\Lambda}^{2} \tau^{-1 / 3} \hat{r}_{0}(\tau)\right)+\mathcal{O}\left(\frac{\widetilde{\Lambda}^{2} \tau^{-1 / 3} \ell_{\tau}^{\infty}}{\hat{u}_{0}(\tau)}\right) \\
& \left(\widetilde{E}_{\tau}(\widetilde{\Lambda})\right)_{12} \underset{\tau \rightarrow+\infty}{=} \mathcal{O}\left(\frac{\widetilde{\Lambda} \tau^{-1 / 3}}{\hat{u}_{0}(\tau)}\right) \\
& \left(\widetilde{E}_{\tau}(\widetilde{\Lambda})\right)_{21} \underset{\tau \rightarrow+\infty}{=} \mathcal{O}\left(\frac{\tau^{-2 / 3} \varkappa_{0}^{2}(\tau)}{\left(\hat{r}_{0}(\tau)\right)^{2}}\right)+\mathcal{O}\left(\tau^{-2 / 3} \varkappa_{0}^{2}(\tau)\right)+\mathcal{O}\left(\frac{\widetilde{\Lambda} \tau^{-2 / 3} \varkappa_{0}^{3}(\tau)}{\left(\hat{r}_{0}(\tau)\right)^{2}}\right)+\mathcal{O}\left(\widetilde{\Lambda} \tau^{-2 / 3} \varkappa_{0}^{3}(\tau)\right) \\
& +\mathcal{O}\left(\frac{\widetilde{\Lambda} \tau^{-1 / 3} \varkappa_{0}^{2}(\tau)}{\hat{u}_{0}(\tau)}\right)+\mathcal{O}\left(\widetilde{\Lambda}^{2} \tau^{-1 / 3} \varkappa_{0}(\tau)\right)+\mathcal{O}\left(\frac{\widetilde{\Lambda}^{2} \tau^{-2 / 3} \ell_{\tau}^{\infty} \varkappa_{0}^{2}(\tau)}{\hat{r}_{0}(\tau) \hat{u}_{0}(\tau)}\right) \\
& +\mathcal{O}\left(\widetilde{\Lambda}^{3} \tau^{-1 / 3}\left(\hat{r}_{0}(\tau)\right)^{2}\right)+\mathcal{O}\left(\widetilde{\Lambda}^{3} \tau^{-1 / 3} \ell_{\tau}^{\infty}\right)+\mathcal{O}\left(\widetilde{\Lambda}^{3} \tau^{-1 / 3} \ell_{\tau}^{\infty} \hat{r}_{0}(\tau)\right) \\
& +\mathcal{O}\left(\frac{\widetilde{\Lambda}^{3} \tau^{-1 / 3}\left(\ell_{\tau}^{\infty}\right)^{2}}{\hat{u}_{0}(\tau)}\right)
\end{aligned}
$$

(vi) One now proceeds to diagonalize the $\widetilde{\Lambda}$ term in Equation (3.111). Set

$$
\mathcal{G}(\tau):=\mathrm{e}^{-\frac{\mathrm{i} \pi}{4}} \sqrt{\varkappa_{0}(\tau)}\left(\begin{array}{cc}
\mathrm{i}\left(\varkappa_{0}(\tau)\right)^{-1} & 0 \\
1 & 1
\end{array}\right) .
$$

Then applying the transformation $F_{6}$, one shows that

$$
\frac{\partial \psi(\widetilde{\Lambda})}{\partial \widetilde{\Lambda}}=\left(B_{0}(\widetilde{\Lambda})+R_{0}(\widetilde{\Lambda})\right) \psi(\widetilde{\Lambda}),
$$


where

$$
B_{0}(\widetilde{\Lambda}):=\mathrm{i} 4 \sqrt{3} \widetilde{\Lambda} \sigma_{3}+\widetilde{q}(\tau) \sigma_{-}+\widetilde{p}(\tau) \sigma_{+}
$$

with $\widetilde{p}(\tau), \widetilde{q}(\tau)$ given in Equations (3.99), and

$$
R_{0}(\widetilde{\Lambda}) \underset{\tau \rightarrow+\infty}{:=} \mathcal{O}\left((\mathcal{G}(\tau))^{-1} \widetilde{E}_{\tau}(\widetilde{\Lambda}) \mathcal{G}(\tau)\right)
$$

where $\widetilde{E}_{\tau}(\widetilde{\Lambda})$ is defined by Equation (3.112).

(vii) Let $\psi_{0}(\widetilde{\Lambda})$ be a fundamental solution of $\frac{\partial \psi_{0}(\widetilde{\Lambda})}{\partial \widetilde{\Lambda}}=B_{0}(\widetilde{\Lambda}) \psi_{0}(\widetilde{\Lambda})$, which coincides with Equation (3.98). Changing variables in Equation (3.98) according to $\mathcal{D}(x):=\psi_{0}(\widetilde{\Lambda})$, where $\widetilde{\Lambda}=x_{0} x$, with $x_{0}=\mathrm{e}^{-\frac{\mathrm{i} \pi}{4}} 2^{-3 / 2} 3^{-1 / 4}$, one proves that $\mathcal{D}(x)$ solves the standard equation

$$
\partial_{x} \mathcal{D}(x)=\left(\frac{x}{2} \sigma_{3}+q^{*}(\tau) \sigma_{-}+p^{*}(\tau) \sigma_{+}\right) \mathcal{D}(x),
$$

where $p^{*}(\tau):=x_{0} \widetilde{p}(\tau)$ and $q^{*}(\tau):=x_{0} \widetilde{q}(\tau)$, with fundamental solution given in terms of the paraboliccylinder function, $D_{*}(\cdot)$ (see, for example, [2, 6, 12]):

$$
\mathcal{D}(x)=\left(\begin{array}{ll}
D_{-1-\nu}(\mathrm{i} x) & D_{\nu}(x) \\
\dot{D}_{-1-\nu}(\mathrm{i} x) & \dot{D}_{\nu}(x)
\end{array}\right)
$$

where $\dot{D}_{*}(z):=\left(p^{*}(\tau)\right)^{-1}\left(\partial_{z} D_{*}(z)-\frac{z}{2} D_{*}(z)\right)$, and

$$
\nu+1:=-p^{*}(\tau) q^{*}(\tau)=-\frac{\mathrm{i}}{8 \sqrt{3}}\left(\varkappa_{0}^{2}(\tau)+\ell_{\tau}^{\infty}+\frac{4\left(a-\frac{\mathrm{i}}{2}\right) \hat{u}_{0}(\tau)}{1+\hat{u}_{0}(\tau)}\right) .
$$

Now, applying definitions (3.65) and (3.97), one re-writes Equation (3.118) as definition (3.91); thus, the representation for $\psi_{0}(\widetilde{\Lambda})$ given in Equations (3.91) and (3.98)-(3.100) is obtained.

Finally, in order to prove the error estimate in Equation (3.94), one has to estimate the function $\chi_{0}(\tilde{\Lambda})$ defined in the transformation $F_{7}$. Applying the transformation $F_{7}$, one re-writes Equation (3.114) as follows:

$$
\frac{\partial \chi_{0}(\widetilde{\Lambda})}{\partial \widetilde{\Lambda}} \underset{\tau \rightarrow+\infty}{=} R_{0}(\widetilde{\Lambda}) \chi_{0}(\widetilde{\Lambda})+\left[B_{0}(\widetilde{\Lambda}), \chi_{0}(\widetilde{\Lambda})\right]
$$

where $B_{0}(\widetilde{\Lambda})$ is defined by Equation (3.115), $\left[B_{0}(\widetilde{\Lambda}), \chi_{0}(\widetilde{\Lambda})\right]:=B_{0}(\widetilde{\Lambda}) \chi_{0}(\widetilde{\Lambda})-\chi_{0}(\widetilde{\Lambda}) B_{0}(\widetilde{\Lambda})$ is the matrix commutator, and $R_{0}(\widetilde{\Lambda})$ is defined by Equation (3.116). The normalized solution $\left(\chi_{0}(0)=\mathrm{I}\right)$ of Equation (3.119) is given by

$$
\chi_{0}(\widetilde{\Lambda})=\mathrm{I}+\int_{0}^{\widetilde{\Lambda}} \psi_{0}(\widetilde{\Lambda})\left(\psi_{0}(\xi)\right)^{-1} R_{0}(\xi) \chi_{0}(\xi) \psi_{0}(\xi)\left(\psi_{0}(\widetilde{\Lambda})\right)^{-1} \mathrm{~d} \xi
$$

To prove the required estimate for $\chi_{0}(\widetilde{\Lambda})$, one uses the method of successive approximations,

$$
\chi_{0}^{(n)}(\widetilde{\Lambda}):=\mathrm{I}+\int_{0}^{\widetilde{\Lambda}} \psi_{0}(\widetilde{\Lambda})\left(\psi_{0}(\xi)\right)^{-1} R_{0}(\xi) \chi_{0}^{(n-1)}(\xi) \psi_{0}(\xi)\left(\psi_{0}(\widetilde{\Lambda})\right)^{-1} \mathrm{~d} \xi, \quad n \in \mathbb{N},
$$

with $\chi_{0}^{(0)}(\widetilde{\Lambda})=\mathrm{I}$, to construct a Neumann series solution for $\chi_{0}(\widetilde{\Lambda})$, that is, $\chi_{0}(\widetilde{\Lambda}):=\lim _{n \rightarrow \infty} \chi_{0}^{(n)}(\widetilde{\Lambda})$. In this case, however, it suffices to estimate the norm of the associated resolvent kernel. Via the above iteration argument, it follows that

$$
\left\|\chi_{0}(\widetilde{\Lambda})-\mathrm{I}\right\| \leqslant \exp \left(\int_{0}^{\widetilde{\Lambda}} \| \psi_{0}(\widetilde{\Lambda})||||\left(\psi_{0}(\xi)\right)^{-1}|||| R_{0}(\xi)|||| \psi_{0}(\xi)||\left|\left(\psi_{0}(\widetilde{\Lambda})\right)^{-1}\right||| \mathrm{d} \xi \mid\right)-1,
$$

where $|\mathrm{d} \xi|$ denotes integration with respect to arc length. One now estimates the norms appearing in Equation (3.121). Using Equations (3.112), (3.113), and (3.116), conditions (3.12), and the conditions of Corollary 3.2.3. one shows that

$$
R_{0}(\xi) \underset{\tau \rightarrow+\infty}{=}\left(\begin{array}{cc}
\mathcal{O}\left(\tau^{2 \varepsilon+\delta-\delta_{1}}\right) & \mathcal{O}\left(\tau^{\varepsilon+\frac{\delta}{2}-\delta_{1}}\right) \\
\mathcal{O}\left(\tau^{3 \varepsilon+\frac{3 \delta}{2}-\delta_{1}}\right) & \mathcal{O}\left(\tau^{2 \varepsilon+\delta-\delta_{1}}\right)
\end{array}\right)
$$


recalling the definition of the matrix norm (cf. Subsection 3.1), one arrives at

$$
\left\|R_{0}(\xi)\right\| \underset{\tau \rightarrow+\infty}{=} \mathcal{O}\left(\tau^{3 \varepsilon+\frac{3 \delta}{2}-\delta_{1}}\right) .
$$

For the function $\psi_{0}(\xi)$, one has to find a uniform approximation for $\xi \in \mathbb{R} \cup i \mathbb{R}$. To do so, one uses the following integral representation for the parabolic-cylinder function [13]:

$$
D_{\nu}(x)=\frac{2^{\frac{\nu}{2}} \mathrm{e}^{-\frac{x^{2}}{4}}}{\Gamma\left(-\frac{\nu}{2}\right)} \int_{0}^{+\infty} \mathrm{e}^{-\frac{t x^{2}}{2}} t^{-\frac{\nu}{2}-1}(1+t)^{\frac{\nu-1}{2}} \mathrm{~d} t, \quad \operatorname{Re}(\nu)<0, \quad|\arg (x)| \leqslant \pi / 4 .
$$

As this integral representation will be applied to the entries of the matrix-valued function $\psi_{0}(\xi)$ (cf. Equation (3.100), it implies the following restriction on $\nu$ :

$$
0 \underset{\tau \rightarrow+\infty}{<} \operatorname{Re}(\nu+1) \underset{\tau \rightarrow+\infty}{<} 1 .
$$

Since, in the sector $|\arg (x)| \leqslant \pi / 4$, the exponents in the integral representation (3.123) are less than or equal to one, the following estimate for $D_{\nu}(x)$ can be deduced:

$$
\left|D_{\nu}(x)\right| \leqslant \frac{\sqrt{\pi} 2^{\operatorname{Re}(\nu) / 2}}{\Gamma\left(\frac{1-\operatorname{Re}(\nu)}{2}\right)}, \quad|\arg (x)| \leqslant \pi / 4 .
$$

From the above inequality, one derives, for the elements of the second column of $\psi_{0}(\xi)$, the following estimates:

$$
\left|\left(\psi_{0}(\xi)\right)_{12}\right| \underset{\tau \rightarrow+\infty}{=} \mathcal{O}(1), \quad\left|\left(\psi_{0}(\xi)\right)_{22}\right| \underset{\tau \rightarrow+\infty}{=} \mathcal{O}(1)+\mathcal{O}\left(\xi\left|\varkappa_{0}(\tau)\right|^{-1}\right), \quad \arg (\xi) \in(-\pi / 2,0) .
$$

In order to apply, simultaneously, the same integral representation (3.123) for the elements of the first column of $\psi_{0}(\xi)$, one has to restrict $\arg (\xi)$ to $-\pi / 2$; hence, one arrives at the following, analogous estimates:

$$
\left|\left(\psi_{0}(\xi)\right)_{11}\right| \underset{\tau \rightarrow+\infty}{=} \mathcal{O}(1), \quad\left|\left(\psi_{0}(\xi)\right)_{21}\right| \underset{\tau \rightarrow+\infty}{=} \mathcal{O}(1)+\mathcal{O}\left(\xi\left|\varkappa_{0}(\tau)\right|^{-1}\right), \quad \arg (\xi)=-\pi / 2 .
$$

Thus, for $\arg (\xi)=-\pi / 2$, one arrives at the following estimate for $\left\|\psi_{0}(\xi)\right\|$ :

$$
\| \psi_{0}(\xi) \mid \underset{\tau \rightarrow+\infty}{=} \mathcal{O}(1)+\mathcal{O}\left(\xi\left|\varkappa_{0}(\tau)\right|^{-1}\right) .
$$

In order to find estimates for $\psi_{0}(\xi)$ on the other Stokes rays $\arg (\xi)=0, \pi / 2, \pm \pi, \ldots$, one has to use the linear relations for the parabolic-cylinder functions relating any three of the four functions $D_{\nu}( \pm x)$ and $D_{-\nu-1}( \pm \mathrm{i} x)$ (see, for example, [11, pg. 1094, Equations 9.248 1.-3.), and impose the additional restriction $\operatorname{Im}(\nu+1)=_{\tau \rightarrow+\infty} \mathcal{O}(1)$, in which case, for all the Stokes rays $\arg (\xi)=$ $0, \pm \pi / 2, \pm \pi, \ldots, 0 \leqslant|\xi|<+\infty$, one verifies an estimate similar to Equation (3.124). Noting that $\operatorname{det}\left(\psi_{0}(\xi)\right)=-\left(p^{*}(\tau)\right)^{-1} \exp \left(-\frac{\mathrm{i} \pi}{2}(\nu+1)\right)$, one obtains

$$
\left\|\left(\psi_{0}(\xi)\right)^{-1}\right\| \underset{\tau \rightarrow+\infty}{=}\left|\varkappa_{0}(\tau)\right| \mathrm{e}^{-\frac{\pi}{2} \operatorname{Im}(\nu+1)}\left\|\psi_{0}(\xi)\right\| .
$$

Using the asymptotic expansions for the parabolic-cylinder functions (see Remark 3.3 .1 below), one shows that

$$
\left\|\psi_{0}(\widetilde{\Lambda})\right\| \underset{\tau \rightarrow+\infty}{=} \mathcal{O}\left(\left|\varkappa_{0}(\tau)\right|^{-1} \widetilde{\Lambda}^{\operatorname{Re}(\nu+1)}\right), \quad\left\|\left(\psi_{0}(\widetilde{\Lambda})\right)^{-1}\right\|_{\tau \rightarrow+\infty}^{=} \mathcal{O}\left(\widetilde{\Lambda}^{\operatorname{Re}(\nu+1)}\right) .
$$

Combining the estimates (3.122), (3.124), (3.125), and (3.126), and assuming that

$$
6 \varepsilon+2 \varepsilon \operatorname{Re}(\nu+1)+\frac{\delta}{2}<\delta_{1},
$$

one deduces from Equation (3.121) that

$$
\left\|\chi_{0}(\widetilde{\Lambda})-\mathrm{I}\right\| \underset{\tau \rightarrow+\infty}{\leqslant} \mathcal{O}\left(\tau^{6 \varepsilon+2 \varepsilon \operatorname{Re}(\nu+1)+\frac{\delta}{2}-\delta_{1}}\right) .
$$


Hence, forming the composition of the invertible linear transformations $F_{1}, \ldots, F_{7}$, that is (the "symbol" o denotes "composition"),

$$
\begin{aligned}
& \widetilde{\Psi}(\widetilde{\mu})=\left(F_{1}^{-1} \circ F_{2}^{-1} \circ F_{3}^{-1} \circ F_{4}^{-1} \circ F_{5}^{-1} \circ F_{6}^{-1} \circ F_{7}^{-1}\right) \psi_{0}(\widetilde{\Lambda}) \\
& =\underbrace{(b(\tau))^{-\frac{1}{2} \sigma_{3}} \mathcal{N}(\tau) \tau^{\frac{1}{6} \sigma_{3}}\left(\begin{array}{cc}
1 & 0 \\
\ell_{\tau} \widetilde{\Lambda} & 1
\end{array}\right) \mathcal{G}(\tau)}_{=: \mathcal{F}_{\tau}(\widetilde{\Lambda})} \chi_{0}(\widetilde{\Lambda}) \psi_{0}(\widetilde{\Lambda}),
\end{aligned}
$$

one arrives at the asymptotic representation for $\widetilde{\Psi}(\widetilde{\mu})$ given in Equation (3.94).

Remark 3.3.1. In Lemma 3.3.1 and hereafter, the matrix-valued function $\psi_{0}(\widetilde{\Lambda})$ (cf. Equation $(3.100)$ ) plays a pivotal role; therefore, for the reader's convenience, its asymptotics are presented here:

$$
\psi_{0}(\widetilde{\Lambda}) \underset{\substack{\widetilde{\Lambda} \rightarrow \infty \\ \arg (\widetilde{\Lambda})=\frac{k \pi}{2}}}{=}\left(\mathrm{I}+\sum_{j=1}^{\infty} \psi_{j}(\tau) \widetilde{\Lambda}^{-j}\right) \exp \left(\left(\mathrm{i} 2 \sqrt{3} \widetilde{\Lambda}^{2}-(\nu+1) \ln \left(\mathrm{e}^{\frac{\mathrm{i} \pi}{4}} 2^{\frac{3}{2}} 3^{\frac{1}{4}} \widetilde{\Lambda}\right)\right) \sigma_{3}\right) \mathcal{R}_{k}, k=-1,0,1,2,
$$

where $\psi_{j}(\tau)$ are off-diagonal (resp., diagonal) matrices for $j$ odd (resp., $j$ even),

$$
\begin{gathered}
\mathcal{R}_{-1}:=\left(\begin{array}{cc}
\mathrm{e}^{-\frac{\pi \mathrm{i}}{2}(\nu+1)} & 0 \\
0 & -\left(p^{*}(\tau)\right)^{-1}
\end{array}\right), \quad \mathcal{R}_{0}:=\left(\begin{array}{cc}
\mathrm{e}^{-\frac{\pi \mathrm{i}}{2}(\nu+1)} & 0 \\
-\frac{\mathrm{i} \sqrt{2 \pi}}{p^{*}(\tau) \Gamma(\nu+1)} \mathrm{e}^{-\frac{\pi \mathrm{i}}{2}(\nu+1)} & -\left(p^{*}(\tau)\right)^{-1}
\end{array}\right), \\
\mathcal{R}_{1}:=\left(\begin{array}{cc}
\mathrm{e}^{\frac{3 \pi \mathrm{i}}{2}(\nu+1)} & \frac{\sqrt{2 \pi}}{\Gamma(-\nu)} \mathrm{e}^{\pi \mathrm{i}(\nu+1)} \\
-\frac{\mathrm{i} \sqrt{2 \pi}}{p^{*}(\tau) \Gamma(\nu+1)} \mathrm{e}^{-\frac{\pi \mathrm{i}}{2}(\nu+1)} & -\left(p^{*}(\tau)\right)^{-1}
\end{array}\right), \quad \mathcal{R}_{2}:=\left(\begin{array}{cc}
\mathrm{e}^{\frac{3 \pi \mathrm{i}}{2}(\nu+1)} & \frac{\sqrt{2 \pi}}{\Gamma(-\nu)} \mathrm{e}^{\pi \mathrm{i}(\nu+1)} \\
0 & -\left(p^{*}(\tau)\right)^{-1} \mathrm{e}^{-2 \pi \mathrm{i}(\nu+1)}
\end{array}\right),
\end{gathered}
$$

and $\Gamma(\cdot)$ is the (Euler) gamma function [1]. The above asymptotic expansion can be deduced from the asymptotics of the parabolic-cylinder functions (see, for example, [13]). The diagonal/off-diagonal structure of the matrices $\psi_{j}(\tau)$ is a consequence of the " $\sigma_{3}$-reduction" for Equation (3.98): $\psi_{0}(\widetilde{\Lambda}) \rightarrow$ $\sigma_{3} \psi_{0}(-\widetilde{\Lambda})$. In Lemmata 3.4.1 and 3.4.2 (see Subsection 3.4 below), explicit knowledge of the following matrices is essential:

$$
\begin{gathered}
\psi_{1}(\tau)=\left(\begin{array}{cc}
0 & \frac{\varkappa_{0}(\tau)}{8 \sqrt{3}} \\
-\frac{\mathrm{i}(\nu+1)}{\varkappa_{0}(\tau)} & 0
\end{array}\right), \quad \psi_{2}(\tau)=\left(\begin{array}{cc}
-\frac{\mathrm{i}(\nu+1)(\nu+2)}{16 \sqrt{3}} & 0 \\
0 & \frac{\mathrm{i} \nu(\nu+1)}{16 \sqrt{3}}
\end{array}\right), \\
\psi_{3}(\tau)=\left(\begin{array}{cc}
0 & \frac{\mathrm{i} \nu(\nu-1) \varkappa_{0}(\tau)}{384} \\
-\frac{(\nu+1)(\nu+2)(\nu+3)}{16 \sqrt{3} \varkappa_{0}(\tau)} & 0
\end{array}\right) .
\end{gathered}
$$

\subsection{Matching of Asymptotics}

In this subsection the connection matrix (cf. Equation (1.11)), $G$, is calculated asymptotically in terms of the matrix elements of the function $\mathcal{A}(\widetilde{\mu}, \tau)$ defined by Equation (3.7), that is, the functions $\hat{r}_{0}(\tau)$, $\hat{u}_{0}(\tau), h_{0}(\tau)$, and $b(\tau)$; thus, under conditions (3.12), the direct monodromy problem for Equation (3.6) is solved asymptotically.

Proposition 3.4.1. Let $\widetilde{\mu}=\widetilde{\mu}_{0}=\alpha+\tau^{-1 / 3} \widetilde{\Lambda}$, where $\widetilde{\Lambda}={ }_{\tau \rightarrow+\infty} \mathcal{O}\left(\tau^{\varepsilon}\right), 0<\varepsilon<1 / 9$. Then under conditions (3.12), with $0<\delta<\varepsilon<1 / 9$ and $\varepsilon<\delta_{1}<1 / 3$,

$$
\begin{aligned}
\left(\mathcal{F}_{\tau}(\widetilde{\Lambda})\right)^{-1} T(\widetilde{\mu}) \underset{\substack{\widetilde{\mu}=\widetilde{\mu}_{0} \\
\tau \rightarrow+\infty}}{=} & \left(\frac{1}{3^{1 / 4}} \sqrt{\frac{\varkappa_{0}(\tau)}{\varpi \widetilde{\Lambda}}}\right)^{\sigma_{3}}\left(\begin{array}{cc}
-\frac{1}{8}\left(1+\mathcal{O}\left(\tau^{\delta-2 \varepsilon}\right)\right) & 1+\mathcal{O}\left(\tau^{\delta-2 \varepsilon}\right) \\
-\frac{1}{2}(\varpi+1)\left(1+\mathcal{O}\left(\tau^{2 \delta-2 \varepsilon}\right)\right) & 4(\varpi-1)\left(1+\mathcal{O}\left(\tau^{2 \delta-2 \varepsilon}\right)\right)
\end{array}\right) \\
& \times\left(\frac{1}{\alpha^{3 / 2}} \sqrt{\frac{b(\tau) \hat{r}_{0}(\tau)}{\hat{u}_{0}(\tau)}}\right)^{\sigma_{3}}
\end{aligned}
$$

where $\varpi$ is defined in Proposition 3.2 .6 . 
Proof. The proof is a straightforward, though algebraically tedious, consequence of Proposition 3.2.6 (cf. Equations (3.88)-(3.90) ) and Lemma 3.3.1 (cf. Equations (3.95)-(3.97)). More precisely, using conditions (3.12), the conditions of Corollary 3.2.3, Equations (3.35) and (3.65), and repeated application of Equation (3.77), one shows that

$$
\begin{aligned}
& \left(\left(\mathcal{F}_{\tau}(\widetilde{\Lambda})\right)^{-1} T\left(\alpha+\tau^{-1 / 3} \widetilde{\Lambda}\right)\right)_{11} \underset{=}{=}-\frac{1}{8 \cdot 3^{1 / 4} \alpha \sqrt{\alpha}} \sqrt{\frac{\hat{r}_{0}(\tau) b(\tau)}{\hat{u}_{0}(\tau)}} \sqrt{\frac{\varkappa_{0}(\tau)}{\varpi \widetilde{\Lambda}}}\left(1+\mathcal{O}\left(h_{0}(\tau) \widetilde{\Lambda}^{-2}\right)\right) \\
& \left(\left(\mathcal{F}_{\tau}(\widetilde{\Lambda})\right)^{-1} T\left(\alpha+\tau^{-1 / 3} \widetilde{\Lambda}\right)\right)_{12}=\frac{\alpha \sqrt{\alpha}}{\tau_{\tau \rightarrow+\infty}^{1 / 4}} \sqrt{\frac{\hat{u}_{0}(\tau)}{\hat{r}_{0}(\tau) b(\tau)}} \sqrt{\frac{\varkappa_{0}(\tau)}{\varpi \widetilde{\Lambda}}}\left(1+\mathcal{O}\left(h_{0}(\tau) \widetilde{\Lambda}^{-2}\right)\right) \\
& \left(\left(\mathcal{F}_{\tau}(\widetilde{\Lambda})\right)^{-1} T\left(\alpha+\tau^{-1 / 3} \widetilde{\Lambda}\right)\right)_{21} \underset{\tau \rightarrow+\infty}{=}-\frac{\sqrt{3}(\varpi+1)}{2 \cdot 3^{1 / 4} \alpha \sqrt{\alpha}} \sqrt{\frac{b(\tau) \hat{r}_{0}(\tau)}{\hat{u}_{0}(\tau)}} \sqrt{\frac{\varpi \widetilde{\Lambda}}{\varkappa_{0}(\tau)}} \\
& \times\left(1+\mathcal{O}\left(\frac{\left(4 \hat{u}_{0}(\tau)+\hat{r}_{0}(\tau)+2\left(\hat{u}_{0}(\tau)\right)^{2}\right) h_{0}(\tau)}{\hat{r}_{0}(\tau)\left(1+\hat{u}_{0}(\tau)\right) \widetilde{\Lambda}^{2}}\right)\right), \\
& \left(\left(\mathcal{F}_{\tau}(\widetilde{\Lambda})\right)^{-1} T\left(\alpha+\tau^{-1 / 3} \widetilde{\Lambda}\right)\right)_{22}=\frac{4 \sqrt{3}(\varpi-1) \alpha \sqrt{\alpha}}{3^{1 / 4}} \sqrt{\frac{\varpi \widetilde{\Lambda}}{\varkappa_{0}(\tau)}} \sqrt{\frac{\hat{u}_{0}(\tau)}{b(\tau) \hat{r}_{0}(\tau)}} \\
& \times\left(1+\mathcal{O}\left(\frac{\left(4 \hat{u}_{0}(\tau)+\hat{r}_{0}(\tau)+2\left(\hat{u}_{0}(\tau)\right)^{2}\right) h_{0}(\tau)}{\hat{r}_{0}(\tau)\left(1+\hat{u}_{0}(\tau)\right) \widetilde{\Lambda}^{2}}\right)\right) ;
\end{aligned}
$$

hence, via conditions (3.12) and the conditions of Corollary 3.2.3, one arrives at, after simplification, Equation (3.127).

Lemma 3.4.1. Let $\widetilde{\Psi}(\widetilde{\mu}, \tau)$ be the fundamental solution of Equation (3.6) with asymptotics given in Lemma 3.3.1, and $Y_{0}^{\infty}(\widetilde{\mu}, \tau)$ be the canonical solution of Equation (3.1). Defin 11

$$
L_{\infty}(\tau):=(\widetilde{\Psi}(\widetilde{\mu}, \tau))^{-1} \tau^{-\frac{1}{12} \sigma_{3}} Y_{0}^{\infty}\left(\tau^{-\frac{1}{6}} \widetilde{\mu}, \tau\right)
$$

Assume that the parameters $\varepsilon, \delta, \delta_{1}$, and $\nu+1$ satisfy the conditions (3.92) and (3.93); furthermore, let the function $b(\tau)$ satisfy the following conditions:

$$
\begin{aligned}
& \left(\frac{\tau^{\frac{1}{3} \operatorname{Im}(a)}\left(\hat{u}_{0}(\tau)\right)^{2}}{b(\tau)\left(\hat{r}_{0}(\tau)\right)^{2}}\right) \tau^{6 \varepsilon+\delta-\delta_{1}} \underset{\tau \rightarrow+\infty}{=} \mathcal{O}\left(\tau^{-\hat{\delta}_{1}}\right),\left(\frac{b(\tau)\left(\hat{r}_{0}(\tau)\right)^{2}}{\tau^{\frac{1}{3} \operatorname{Im}(a)}\left(\hat{u}_{0}(\tau)\right)^{2}}\right) \tau^{6 \varepsilon+4 \varepsilon \operatorname{Re}(\nu+1)-\delta_{1}} \underset{\tau \rightarrow+\infty}{=} \mathcal{O}\left(\tau^{-\hat{\delta}_{2}}\right) \\
& \left(\frac{\tau^{\frac{1}{3} \operatorname{Im}(a)}\left(\hat{u}_{0}(\tau)\right)^{2}}{b(\tau)\left(\hat{r}_{0}(\tau)\right)^{2}}\right) \tau^{-\varepsilon-2 \varepsilon \operatorname{Re}(\nu+1)} \underset{\tau \rightarrow+\infty}{=} \mathcal{O}\left(\tau^{-\hat{\delta}_{3}}\right),\left(\frac{b(\tau)\left(\hat{r}_{0}(\tau)\right)^{2}}{\tau^{\frac{1}{3} \operatorname{Im}(a)}\left(\hat{u}_{0}(\tau)\right)^{2}}\right) \tau^{2 \delta+2 \varepsilon \operatorname{Re}(\nu+1)-3 \varepsilon} \underset{\tau \rightarrow+\infty}{=} \mathcal{O}\left(\tau^{-\hat{\delta}_{4}}\right),
\end{aligned}
$$

where $\hat{\delta}_{k}>0, k=1,2,3,4$. Then

$$
\begin{aligned}
L_{\infty}(\tau) \underset{\tau \rightarrow+\infty}{=} & \mathrm{i} \mathcal{R}_{0}^{-1} \exp \left(\left(\mathfrak{t}_{\infty}+\mathfrak{c}_{\infty}-\mathcal{I}_{\infty}^{\sharp}(\tau)\right) \sigma_{3}\right)\left(\sqrt{\frac{\varkappa_{0}(\tau) \hat{u}_{0}(\tau)}{b(\tau) \hat{r}_{0}(\tau)}}\right)^{\sigma_{3}} \sigma_{2} \\
& \times\left(\mathrm{I}+\left(\begin{array}{cc}
\mathcal{O}\left(\tau^{-\delta_{11}}\right) & \mathcal{O}\left(\tau^{-\delta_{12}}\right) \\
\mathcal{O}\left(\tau^{-\delta_{21}}\right) & \mathcal{O}\left(\tau^{-\delta_{22}}\right)
\end{array}\right)\right),
\end{aligned}
$$

where $\mathcal{R}_{0}$ is defined in Remark 3.3.1, $\mathcal{I}_{\infty}^{\sharp}(\tau)$ is defined by Equation (3.59),

$$
\mathfrak{t}_{\infty}:=\mathrm{i} 3(\sqrt{3}-1) \alpha^{2} \tau^{2 / 3}+\frac{1}{3}\left(\nu+\frac{1}{2}-\frac{\mathrm{i} a}{2}\right) \ln \tau,
$$

\footnotetext{
${ }^{11}$ Note that $\tau^{-\frac{1}{12} \sigma_{3}} Y_{0}^{\infty}\left(\tau^{-\frac{1}{6}} \widetilde{\mu}, \tau\right)$ is also a fundamental solution of Equation [3.6]; therefore, $L_{\infty}(\tau)$ is independent of $\widetilde{\mu}$.
} 


$$
\mathfrak{c}_{\infty}:=\frac{3}{2} \ln \alpha-\frac{1}{4} \ln 3+(\nu+1) \ln \left(\mathrm{e}^{\frac{\mathbf{i} \pi}{4}} 2^{3 / 2} 3^{1 / 4}\right)+C_{\infty}^{\mathrm{WKB}},
$$

with $C_{\infty}^{\mathrm{WKB}}$ defined by Equation (3.55), and $\delta_{11}^{\infty}=\delta_{22}^{\infty}:=\min \left\{\delta_{1}-\frac{\delta}{2}-6 \varepsilon-2 \varepsilon \operatorname{Re}(\nu+1), 2(\varepsilon-\delta), \frac{1}{3}-3 \varepsilon\right\}$, $\delta_{12}^{\infty}:=\min \left\{\hat{\delta}_{1}, \hat{\delta}_{3}\right\}$, and $\delta_{21}^{\infty}:=\min \left\{\hat{\delta}_{2}, \hat{\delta}_{4}\right\}$.

Proof. Denote by $\widetilde{\Psi}_{\mathrm{WKB}}(\widetilde{\mu}, \tau)$ the solution of Equation (3.6) which has WKB asymptotics given by Equations (3.18)- (3.20) in the canonical domain containing the Stokes curve approaching the positive real $\widetilde{\mu}$-axis from above as $\widetilde{\mu} \rightarrow+\infty$. Now, re-write $L_{\infty}(\tau)$ in the following, equivalent form:

$$
L_{\infty}(\tau)=\left((\widetilde{\Psi}(\widetilde{\mu}, \tau))^{-1} \widetilde{\Psi}_{\mathrm{WKB}}(\widetilde{\mu}, \tau)\right)\left(\left(\widetilde{\Psi}_{\mathrm{WKB}}(\widetilde{\mu}, \tau)\right)^{-1} \tau^{-\frac{1}{12} \sigma_{3}} Y_{0}^{\infty}\left(\tau^{-\frac{1}{6}} \widetilde{\mu}, \tau\right)\right) .
$$

Noting that $\widetilde{\Psi}(\widetilde{\mu}, \tau), \widetilde{\Psi}_{\mathrm{WKB}}(\widetilde{\mu}, \tau)$, and $\tau^{-\frac{1}{12} \sigma_{3}} Y_{0}^{\infty}\left(\tau^{-\frac{1}{6}} \widetilde{\mu}, \tau\right)$ are all solutions of Equation (3.6), it follows that they differ by right-hand, $\widetilde{\mu}$-independent matrix factors. Using this fact, one evaluates asymptotically (as $\tau \rightarrow+\infty$ ) each pair by taking separate limits, that is, $\widetilde{\mu} \rightarrow \alpha$ and $\widetilde{\mu} \rightarrow+\infty$, respectively; more precisely,

$$
\begin{gathered}
(\widetilde{\Psi}(\widetilde{\mu}, \tau))^{-1} \widetilde{\Psi}_{\mathrm{WKB}}(\widetilde{\mu}, \tau) \underset{\tau \rightarrow+\infty}{=} \underbrace{6 \varepsilon+2 \varepsilon \operatorname{Re}(\nu+1)+\frac{\delta}{2}-\delta_{1}}_{\widetilde{\mu}=\widetilde{\mu}_{0}=\alpha+\tau^{-1 / 3} \widetilde{\Lambda}, \quad \mathcal{I}_{\tau}(\widetilde{\Lambda})\left(\mathrm{I}+\mathcal{O}\left(\tau^{\varepsilon}, 0<\varepsilon<1 / 9, \quad \arg (\widetilde{\Lambda})=0\right.\right.})) \psi_{0}(\widetilde{\Lambda}))^{-1} T(\widetilde{\mu}) \mathrm{e}^{\mathrm{WKB}(\widetilde{\mu}, \tau)}, \\
\left(\widetilde{\Psi}_{\mathrm{WKB}}(\widetilde{\mu}, \tau)\right)^{-1} \tau^{-\frac{1}{12} \sigma_{3}} Y_{0}^{\infty}\left(\tau^{-\frac{1}{6}} \widetilde{\mu}, \tau\right) \underset{\tau \rightarrow+\infty}{=} \underbrace{\left(T(\widetilde{\mu}) \mathrm{e}^{\mathrm{WKB}(\widetilde{\mu}, \tau)}\right)^{-1} \tau^{-\frac{1}{12} \sigma_{3}} Y_{0}^{\infty}\left(\tau^{-\frac{1}{6}} \widetilde{\mu}, \tau\right)}_{\widetilde{\mu} \rightarrow \infty, \quad \arg (\widetilde{\mu})=0},
\end{gathered}
$$

where $\operatorname{WKB}(\widetilde{\mu}, \tau):=-\sigma_{3} \mathrm{i} \tau^{2 / 3} \int_{\widetilde{\mu}_{0}}^{\widetilde{\mu}} l(\xi) \mathrm{d} \xi-\int_{\widetilde{\mu}_{0}}^{\widetilde{\mu}} \operatorname{diag}\left((T(\xi))^{-1} \partial_{\xi} T(\xi)\right) \mathrm{d} \xi$.

To calculate asymptotics of $(\widetilde{\Psi}(\widetilde{\mu}, \tau))^{-1} \widetilde{\Psi}_{\mathrm{WKB}}(\widetilde{\mu}, \tau)$, one uses Equation (3.127), with $\varpi=+1$, and Equation (3.100) (in conjunction with Remark 3.3.1) to show that

$$
\begin{aligned}
& (\widetilde{\Psi}(\widetilde{\mu}, \tau))^{-1} \widetilde{\Psi}_{\mathrm{WKB}}(\widetilde{\mu}, \tau) \underset{\tau \rightarrow+\infty}{=}\left(\mathrm{I}+\left(\psi_{0}(\widetilde{\Lambda})\right)^{-1} \mathcal{O}\left(\tau^{6 \varepsilon+2 \varepsilon \operatorname{Re}(\nu+1)+\frac{\delta}{2}-\delta_{1}}\right) \psi_{0}(\widetilde{\Lambda})\right)\left(\psi_{0}(\widetilde{\Lambda})\right)^{-1}\left(\mathcal{F}_{\tau}(\widetilde{\Lambda})\right)^{-1} T(\widetilde{\mu}) \\
& \underset{\tau \rightarrow+\infty}{=}\left(\mathrm{I}+\left(\psi_{0}(\widetilde{\Lambda})\right)^{-1} \mathcal{O}\left(\tau^{6 \varepsilon+2 \varepsilon \operatorname{Re}(\nu+1)+\frac{\delta}{2}-\delta_{1}}\right) \psi_{0}(\widetilde{\Lambda})\right) \mathcal{R}_{0}^{-1} \\
& \times \exp \left(-\left(\mathrm{i} 2 \sqrt{3} \widetilde{\Lambda}^{2}-(\nu+1) \ln \left(\mathrm{e}^{\frac{\mathrm{i} \pi}{4}} 2^{3 / 2} 3^{1 / 4} \widetilde{\Lambda}\right)\right) \sigma_{3}\right) \\
& \times\left(\left(\begin{array}{cc}
1 & -\frac{\varkappa_{0}(\tau)}{8 \sqrt{3} \Lambda} \\
0 & 1
\end{array}\right)+\frac{1}{\widetilde{\Lambda}}\left(\begin{array}{cc}
0 & 0 \\
\frac{\mathrm{i}(\nu+1)}{\varkappa_{0}(\tau)} & 0
\end{array}\right)+\frac{1}{\widetilde{\Lambda}^{2}}\left(\begin{array}{cc}
\frac{\mathrm{i} \nu(\nu+1)}{16 \sqrt{3}} & 0 \\
0 & -\frac{\mathrm{i}(\nu+1)(\nu+2)}{16 \sqrt{3}}
\end{array}\right)\right. \\
& \left.+\frac{1}{\widetilde{\Lambda}^{3}}\left(\begin{array}{cc}
0 & -\frac{\mathrm{i} \nu(\nu-1) \varkappa_{0}(\tau)}{384} \\
\frac{(\nu+1)(\nu+2)(\nu+3)}{16 \sqrt{3} \varkappa_{0}(\tau)} & 0
\end{array}\right)+\mathcal{O}\left(\frac{1}{\widetilde{\Lambda}^{4}}\left(\begin{array}{cc}
\mathcal{O}(1) & 0 \\
0 & \mathcal{O}(1)
\end{array}\right)\right)\right) \\
& \times\left(\frac{1}{3^{1 / 4}} \sqrt{\frac{\varkappa_{0}(\tau)}{\widetilde{\Lambda}}}\right)^{\sigma_{3}}\left(\left(\begin{array}{cc}
-\frac{1}{8} & 1 \\
-1 & 0
\end{array}\right)+\left(\begin{array}{cc}
\mathcal{O}\left(\tau^{\delta-2 \varepsilon}\right) & \mathcal{O}\left(\tau^{\delta-2 \varepsilon}\right) \\
\mathcal{O}\left(\tau^{2 \delta-2 \varepsilon}\right) & 0
\end{array}\right)\right) \\
& \times\left(\frac{1}{\alpha^{3 / 2}} \sqrt{\frac{b(\tau) \hat{r}_{0}(\tau)}{\hat{u}_{0}(\tau)}}\right)^{\sigma_{3}} \\
& \underset{\tau \rightarrow+\infty}{=}\left(\mathrm{I}+\left(\psi_{0}(\widetilde{\Lambda})\right)^{-1} \mathcal{O}\left(\tau^{6 \varepsilon+2 \varepsilon \operatorname{Re}(\nu+1)+\frac{\delta}{2}-\delta_{1}}\right) \psi_{0}(\widetilde{\Lambda})\right) \mathcal{R}_{0}^{-1} \\
& \times\left(\frac{\alpha^{3 / 2}}{3^{1 / 4}} \sqrt{\frac{\varkappa_{0}(\tau) \hat{u}_{0}(\tau)}{b(\tau) \hat{r}_{0}(\tau) \widetilde{\Lambda}}}\right)^{\sigma_{3}} \exp \left(-\left(\mathrm{i} 2 \sqrt{3} \widetilde{\Lambda}^{2}-(\nu+1) \ln \left(\mathrm{e}^{\mathrm{i} \pi} 2^{3 / 2} 3^{1 / 4} \widetilde{\Lambda}\right)\right) \sigma_{3}\right) \mathrm{i} \sigma_{2} \\
& \times\left(\operatorname{I}+\tau^{-2 \varepsilon}\left(\begin{array}{cc}
\mathcal{O}\left(\tau^{2 \delta}\right) & \mathcal{O}\left(\frac{\hat{u}_{0}(\tau)}{b(\tau) \hat{r}_{0}(\tau)}\right) \\
\mathcal{O}\left(\frac{b(\tau) \hat{r}_{0}(\tau) \tau^{2 \delta}}{\hat{u}_{0}(\tau)}\right) & \mathcal{O}\left(\tau^{\delta}\right)
\end{array}\right)\right) .
\end{aligned}
$$

For the calculation of asymptotics for $\left(\widetilde{\Psi}_{\mathrm{WKB}}(\widetilde{\mu}, \tau)\right)^{-1} \tau^{-\frac{1}{12} \sigma_{3}} Y_{0}^{\infty}\left(\tau^{-\frac{1}{6}} \widetilde{\mu}, \tau\right)$, one uses the asymptotics of $Y_{0}^{\infty}\left(\tau^{-\frac{1}{6}} \widetilde{\mu}, \tau\right)$ given in Proposition 1.3. Equation (3.86), Equations (3.54) and (3.55), Equations (3.58) and (3.59), and conditions (3.93), to show that

$$
\left(\widetilde{\Psi}_{\mathrm{WKB}}(\widetilde{\mu}, \tau)\right)^{-1} \tau^{-\frac{1}{12} \sigma_{3}} Y_{0}^{\infty}\left(\tau^{-\frac{1}{6}} \widetilde{\mu}, \tau\right) \underset{\tau \rightarrow+\infty}{=} \mathrm{e}^{\widetilde{\mathcal{W}}_{\infty}(\tau) \sigma_{3}}\left(\mathrm{I}+\mathcal{O}\left(\tau^{-2(\varepsilon-\delta)}\right)+\mathcal{O}\left(\tau^{-\frac{1}{3}+3 \varepsilon}\right)\right),
$$


where

$$
\begin{aligned}
\widetilde{\mathcal{W}}_{\infty}(\tau) \underset{\substack{:=\\
\tau \rightarrow+\infty}}{ }-\mathrm{i} 3(\sqrt{3}-1) \alpha^{2} \tau^{2 / 3}-\mathrm{i} 2 \sqrt{3} \widetilde{\Lambda}^{2}+\frac{\mathrm{i} a}{6} \ln \tau+\mathcal{I}_{\infty}^{\sharp}(\tau)-C_{\infty}^{\mathrm{WKB}} \\
+\frac{\mathrm{i}}{2 \sqrt{3}}\left(\left(a-\frac{\mathrm{i}}{2}\right)+\frac{h_{0}(\tau)}{\alpha^{2}}-\frac{\mathfrak{p}_{\tau}}{4}\right)\left(\frac{1}{3} \ln \tau-\ln \widetilde{\Lambda}\right) .
\end{aligned}
$$

Hence, via expansions (3.133) and (3.134), upon taking into account definition (3.91), and conditions (3.92) and (3.93), one arrives at

$$
L_{\infty}(\tau) \underset{\tau \rightarrow+\infty}{=} \mathrm{i} \mathcal{R}_{0}^{-1} \exp \left(\left(\mathfrak{t}_{\infty}+\mathfrak{c}_{\infty}-\mathcal{I}_{\infty}^{\sharp}(\tau)\right) \sigma_{3}\right)\left(\sqrt{\frac{\varkappa_{0}(\tau) \hat{u}_{0}(\tau)}{b(\tau) \hat{r}_{0}(\tau)}}\right)^{\sigma_{3}} \sigma_{2}\left(\mathrm{I}+E_{\infty}(\tau)\right),
$$

where $\mathfrak{t}_{\infty}$ and $\mathfrak{c}_{\infty}$ are defined by Equations (3.131) and (3.132), respectively, and

$$
\begin{aligned}
& E_{\infty}(\tau) \underset{\tau \rightarrow+\infty}{=} \tau^{6 \varepsilon+2 \varepsilon \operatorname{Re}(\nu+1)+\frac{\delta}{2}-\delta_{1}}\left(\begin{array}{cc}
\mathcal{O}(1) & \mathcal{O}\left(\frac{\varkappa_{0}(\tau)\left(\hat{u}_{0}(\tau)\right)^{2} \mathrm{e}^{\eta_{1}(\tau)}}{b(\tau)\left(\hat{r}_{0}(\tau)\right)^{2}}\right) \\
\mathcal{O}\left(\frac{b(\tau)\left(\hat{r}_{0}(\tau)\right)^{2} \mathrm{e}^{-\eta_{1}(\tau)}}{\varkappa_{0}(\tau)\left(\hat{u}_{0}(\tau)\right)^{2}}\right) & \mathcal{O}(1)
\end{array}\right) \\
&+\tau^{-2 \varepsilon}\left(\begin{array}{cc}
\mathcal{O}\left(\tau^{2 \delta}\right) & \mathcal{O}\left(\frac{\left(\hat{u}_{0}(\tau)\right)^{2} \tau^{\varepsilon} \eta^{\eta_{1}(\tau)}}{b(\tau)\left(\hat{r}_{0}(\tau)\right)^{2}}\right) \\
\mathcal{O}\left(\frac{\left(\hat{r}_{0}(\tau)\right)^{2} b(\tau) \tau^{2 \delta} \tau^{-\varepsilon} \mathrm{e}^{-\eta_{1}(\tau)}}{\left(\hat{u}_{0}(\tau)\right)^{2}}\right) & \mathcal{O}\left(\tau^{\delta}\right)
\end{array}\right) \\
&+\left(\mathcal{O}\left(\tau^{-2(\varepsilon-\delta)}\right)+\mathcal{O}\left(\tau^{-\frac{1}{3}+3 \varepsilon}\right)\right)\left(\begin{array}{cc}
\mathcal{O}(1) & o(1) \\
o(1) & \mathcal{O}(1)
\end{array}\right),
\end{aligned}
$$

where $\eta_{1}(\tau):=\frac{1}{3} \operatorname{Im}(a) \ln \tau-2 \varepsilon \operatorname{Re}(\nu+1) \ln \tau$. Invoking conditions (3.128) and (3.129), and conditions (3.92) and (3.93), one arrives at Equation (3.130).

Lemma 3.4.2. Let $\widetilde{\Psi}(\widetilde{\mu}, \tau)$ be the fundamental solution of Equation (3.6) with asymptotics given in Lemma 3.3.1, and $X_{0}^{0}(\widetilde{\mu}, \tau)$ be the canonical solution of Equation (3.1). Defin 12

$$
L_{0}(\tau):=(\widetilde{\Psi}(\widetilde{\mu}, \tau))^{-1} \tau^{-\frac{1}{12} \sigma_{3}} X_{0}^{0}\left(\tau^{-\frac{1}{6}} \widetilde{\mu}, \tau\right) .
$$

Assume that the parameters $\varepsilon, \delta, \delta_{1}$, and $\nu+1$ satisfy the conditions (3.92) and (3.93); furthermore, let

$$
6 \varepsilon+\delta-\delta_{1}<0, \quad 6 \varepsilon+4 \varepsilon \operatorname{Re}(\nu+1)-\delta_{1}<0, \quad-3 \varepsilon+2 \varepsilon \operatorname{Re}(\nu+1)+2 \delta<0 .
$$

Then

$$
\begin{aligned}
L_{0}(\tau) \underset{\tau \rightarrow+\infty}{=} & -\mathrm{i} \mathcal{R}_{2}^{-1} \exp \left(\left(\mathfrak{t}_{0}+\mathfrak{c}_{0}+\mathcal{I}_{0}^{\sharp}(\tau)\right) \sigma_{3}\right)\left(\sqrt{\frac{\varkappa_{0}(\tau) \hat{r}_{0}(\tau)}{\hat{u}_{0}(\tau)}}\right)^{\sigma_{3}} \sigma_{2} \\
& \times\left(\mathrm{I}+\left(\begin{array}{ll}
\mathcal{O}\left(\tau^{-\delta_{11}^{0}}\right) & \mathcal{O}\left(\tau^{-\delta_{12}^{0}}\right) \\
\mathcal{O}\left(\tau^{-\delta_{21}^{0}}\right) & \mathcal{O}\left(\tau^{-\delta_{22}^{0}}\right)
\end{array}\right)\right),
\end{aligned}
$$

where $\mathcal{R}_{2}$ is defined in Remark 3.3.1, $\mathcal{I}_{0}^{\sharp}(\tau)$ is defined by Equation (3.61),

$$
\begin{gathered}
\mathfrak{t}_{0}:=\mathrm{i} 3 \sqrt{3} \alpha^{2} \tau^{2 / 3}+\frac{1}{3}\left(\nu+\frac{1}{2}\right) \ln \tau, \\
\mathfrak{c}_{0}:=-\frac{9}{4} \ln 2-\frac{1}{4} \ln 3+(\nu+1) \ln \left(\mathrm{e}^{\frac{\mathrm{i} \pi}{4}} 2^{3 / 2} 3^{1 / 4}\right)-C_{0}^{\mathrm{WKB}},
\end{gathered}
$$

with $C_{0}^{\mathrm{WKB}}$ defined by Equation (3.57), and $\delta_{11}^{0}=\delta_{22}^{0}:=\min \left\{\delta_{1}-\frac{\delta}{2}-6 \varepsilon-2 \varepsilon \operatorname{Re}(\nu+1), 2(\varepsilon-\delta), \frac{1}{3}-3 \varepsilon\right\}$, $\delta_{12}^{0}:=\min \left\{\delta_{1}-\delta-6 \varepsilon, \varepsilon+2 \varepsilon \operatorname{Re}(\nu+1)\right\}$, and $\delta_{21}^{0}:=\min \left\{\delta_{1}-6 \varepsilon-4 \varepsilon \operatorname{Re}(\nu+1), 3 \varepsilon-2 \delta-2 \varepsilon \operatorname{Re}(\nu+1)\right\}$. $\widetilde{\mu}$.

${ }^{12}$ Note that $\tau^{-\frac{1}{12} \sigma_{3}} X_{0}^{0}\left(\tau^{-\frac{1}{6}} \tilde{\mu}, \tau\right)$ is also a fundamental solution of Equation (3.6); therefore, $L_{0}(\tau)$ is independent of 
Proof. Denote by $\widetilde{\Psi}_{\mathrm{WKB}}(\widetilde{\mu}, \tau)$ the solution of Equation (3.6) which has WKB asymptotics given by Equations (3.18)-(3.20) in the canonical domain containing the Stokes curve approaching the positive real $\widetilde{\mu}$-axis from above as $\widetilde{\mu} \rightarrow+0$. Now, re-write $L_{0}(\tau)$ in the following, equivalent form:

$$
L_{0}(\tau)=\left((\widetilde{\Psi}(\widetilde{\mu}, \tau))^{-1} \widetilde{\Psi}_{\mathrm{WKB}}(\widetilde{\mu}, \tau)\right)\left(\left(\widetilde{\Psi}_{\mathrm{WKB}}(\widetilde{\mu}, \tau)\right)^{-1} \tau^{-\frac{1}{12} \sigma_{3}} X_{0}^{0}\left(\tau^{-\frac{1}{6}} \widetilde{\mu}, \tau\right)\right) .
$$

Noting that $\widetilde{\Psi}(\widetilde{\mu}, \tau), \widetilde{\Psi}_{\mathrm{WKB}}(\widetilde{\mu}, \tau)$, and $\tau^{-\frac{1}{12} \sigma_{3}} X_{0}^{0}\left(\tau^{-\frac{1}{6}} \widetilde{\mu}, \tau\right)$ are all solutions of Equation (3.6), it follows that they differ by right-hand, $\widetilde{\mu}$-independent matrix factors. Using this fact, one evaluates asymptotically (as $\tau \rightarrow+\infty$ ) each pair by taking separate limits, that is, $\widetilde{\mu} \rightarrow \alpha$ and $\widetilde{\mu} \rightarrow+0$, respectively; more precisely,

$$
\begin{gathered}
(\widetilde{\Psi}(\widetilde{\mu}, \tau))^{-1} \widetilde{\Psi}_{\mathrm{WKB}}(\widetilde{\mu}, \tau) \underset{\tau \rightarrow+\infty}{=} \underbrace{\left(\mathcal{F}_{\tau}(\widetilde{\Lambda})\left(\mathrm{I}+\mathcal{O}\left(\tau^{6 \varepsilon+2 \varepsilon \operatorname{Re}(\nu+1)+\frac{\delta}{2}-\delta_{1}}\right)\right) \psi_{0}(\widetilde{\Lambda})\right)^{-1} T(\widetilde{\mu}) \mathrm{e}^{\mathrm{WKB}(\widetilde{\mu}, \tau)}}_{\widetilde{\mu}=\widetilde{\mu}_{0}=\alpha+\tau^{-1 / 3} \widetilde{\Lambda}, \quad \tilde{\Lambda} \sim \tau^{\varepsilon}, 0<\varepsilon<1 / 9, \quad \arg (\widetilde{\Lambda})=\pi}, \\
\left(\widetilde{\Psi}_{\mathrm{WKB}}(\widetilde{\mu}, \tau)\right)^{-1} \tau^{-\frac{1}{12} \sigma_{3}} X_{0}^{0}\left(\tau^{-\frac{1}{6}} \widetilde{\mu}, \tau\right) \underset{\tau \rightarrow+\infty}{=} \underbrace{\left(T(\widetilde{\mu}) \mathrm{e}^{\mathrm{WKB}(\widetilde{\mu}, \tau)}\right)^{-1} \tau^{-\frac{1}{12} \sigma_{3}} X_{0}^{0}\left(\tau^{-\frac{1}{6}} \widetilde{\mu}, \tau\right)}_{\widetilde{\mu} \rightarrow 0, \quad \arg (\widetilde{\mu})=0},
\end{gathered}
$$

where $\operatorname{WKB}(\widetilde{\mu}, \tau):=-\sigma_{3} \mathrm{i} \tau^{2 / 3} \int_{\widetilde{\mu}_{0}}^{\widetilde{\mu}} l(\xi) \mathrm{d} \xi-\int_{\widetilde{\mu}_{0}}^{\widetilde{\mu}} \operatorname{diag}\left((T(\xi))^{-1} \partial_{\xi} T(\xi)\right) \mathrm{d} \xi$.

To calculate asymptotics of $(\widetilde{\Psi}(\widetilde{\mu}, \tau))^{-1} \widetilde{\Psi}_{\mathrm{WKB}}(\widetilde{\mu}, \tau)$, one uses Equation (3.127), with $\varpi=-1$, and Equation (3.100) (in conjunction with Remark 3.3.1) to show that

$$
\begin{aligned}
& (\widetilde{\Psi}(\widetilde{\mu}, \tau))^{-1} \widetilde{\Psi}_{\mathrm{WKB}}(\widetilde{\mu}, \tau) \underset{\tau \rightarrow+\infty}{=}\left(\mathrm{I}+\left(\psi_{0}(\widetilde{\Lambda})\right)^{-1} \mathcal{O}\left(\tau^{6 \varepsilon+2 \varepsilon \operatorname{Re}(\nu+1)+\frac{\delta}{2}-\delta_{1}}\right) \psi_{0}(\widetilde{\Lambda})\right)\left(\psi_{0}(\widetilde{\Lambda})\right)^{-1}\left(\mathcal{F}_{\tau}(\widetilde{\Lambda})\right)^{-1} T(\widetilde{\mu}) \\
& \underset{\tau \rightarrow+\infty}{=}\left(\mathrm{I}+\left(\psi_{0}(\widetilde{\Lambda})\right)^{-1} \mathcal{O}\left(\tau^{6 \varepsilon+2 \varepsilon \operatorname{Re}(\nu+1)+\frac{\delta}{2}-\delta_{1}}\right) \psi_{0}(\widetilde{\Lambda})\right) \mathcal{R}_{2}^{-1} \\
& \times \exp \left(-\left(\mathrm{i} 2 \sqrt{3} \widetilde{\Lambda}^{2}-(\nu+1) \ln \left(\mathrm{e}^{\mathrm{i} \pi} \frac{1}{4} 2^{3 / 2} 3^{1 / 4} \widetilde{\Lambda}\right)\right) \sigma_{3}\right) \\
& \times\left(\left(\begin{array}{cc}
1 & -\frac{\varkappa_{0}(\tau)}{8 \sqrt{3} \Lambda} \\
0 & 1
\end{array}\right)+\frac{1}{\widetilde{\Lambda}}\left(\begin{array}{cc}
0 & 0 \\
\frac{\mathrm{i}(\nu+1)}{\varkappa_{0}(\tau)} & 0
\end{array}\right)+\frac{1}{\widetilde{\Lambda}^{2}}\left(\begin{array}{cc}
\frac{\mathrm{i} \nu(\nu+1)}{16 \sqrt{3}} & 0 \\
0 & -\frac{\mathrm{i}(\nu+1)(\nu+2)}{16 \sqrt{3}}
\end{array}\right)\right. \\
& \left.+\frac{1}{\widetilde{\Lambda}^{3}}\left(\begin{array}{cc}
0 & -\frac{\mathrm{i} \nu(\nu-1) \varkappa_{0}(\tau)}{384} \\
\frac{(\nu+1)(\nu+2)(\nu+3)}{16 \sqrt{3} \varkappa_{0}(\tau)} & 0
\end{array}\right)+\mathcal{O}\left(\frac{1}{\widetilde{\Lambda}^{4}}\left(\begin{array}{cc}
\mathcal{O}(1) & 0 \\
0 & \mathcal{O}(1)
\end{array}\right)\right)\right) \\
& \times\left(\frac{\mathrm{e}^{-\frac{\mathrm{i} \pi}{2}}}{3^{1 / 4}} \sqrt{\frac{\varkappa_{0}(\tau)}{\widetilde{\Lambda}}}\right)^{\sigma_{3}}\left(\left(\begin{array}{cc}
-\frac{1}{8} & 1 \\
0 & -8
\end{array}\right)+\left(\begin{array}{cc}
\mathcal{O}\left(\tau^{\delta-2 \varepsilon}\right) & \mathcal{O}\left(\tau^{\delta-2 \varepsilon}\right) \\
0 & \mathcal{O}\left(\tau^{2 \delta-2 \varepsilon}\right)
\end{array}\right)\right) \\
& \times\left(\frac{1}{\alpha^{3 / 2}} \sqrt{\frac{b(\tau) \hat{r}_{0}(\tau)}{\hat{u}_{0}(\tau)}}\right)^{\sigma_{3}} \\
& \underset{\tau \rightarrow+\infty}{=}-\left(\mathrm{I}+\left(\psi_{0}(\widetilde{\Lambda})\right)^{-1} \mathcal{O}\left(\tau^{6 \varepsilon+2 \varepsilon \operatorname{Re}(\nu+1)+\frac{\delta}{2}-\delta_{1}}\right) \psi_{0}(\widetilde{\Lambda})\right) \\
& \times \mathcal{R}_{2}^{-1}\left(\frac{-\mathrm{i}}{8 \cdot 3^{1 / 4} \alpha^{3 / 2}} \sqrt{\frac{b(\tau) \varkappa_{0}(\tau) \hat{r}_{0}(\tau)}{\hat{u}_{0}(\tau) \widetilde{\Lambda}}}\right)^{\sigma_{3}} \\
& \times \exp \left(-\left(\mathrm{i} 2 \sqrt{3} \widetilde{\Lambda}^{2}-(\nu+1) \ln \left(\mathrm{e}^{\mathrm{i} \pi} 2^{3 / 2} 3^{1 / 4} \widetilde{\Lambda}\right)\right) \sigma_{3}\right) \\
& \times\left(\operatorname{I}+\tau^{-2 \varepsilon}\left(\begin{array}{cc}
\mathcal{O}\left(\tau^{\delta}\right) & \mathcal{O}\left(\frac{\hat{u}_{0}(\tau) \tau^{2 \delta}}{b(\tau) \hat{r}_{0}(\tau)}\right) \\
\mathcal{O}\left(\frac{b(\tau) \hat{r}_{0}(\tau)}{\hat{u}_{0}(\tau)}\right) & \mathcal{O}\left(\tau^{2 \delta}\right)
\end{array}\right)\right)
\end{aligned}
$$

For the calculation of asymptotics for $\left(\widetilde{\Psi}_{\mathrm{WKB}}(\widetilde{\mu}, \tau)\right)^{-1} \tau^{-\frac{1}{12} \sigma_{3}} X_{0}^{0}\left(\tau^{-\frac{1}{6}} \widetilde{\mu}, \tau\right)$, one uses the asymptotics of $X_{0}^{0}\left(\tau^{-\frac{1}{6}} \widetilde{\mu}, \tau\right)$ given in Proposition 1.3, Equation (3.87), Equations (3.56) and (3.57), Equations (3.60) and (3.61), and conditions (3.93), to show that

$$
\begin{aligned}
& \left(\widetilde{\Psi}_{\mathrm{WKB}}(\widetilde{\mu}, \tau)\right)^{-1} \tau^{-\frac{1}{12} \sigma_{3}} X_{0}^{0}\left(\tau^{-\frac{1}{6}} \widetilde{\mu}, \tau\right) \underset{\tau \rightarrow+\infty}{=} \mathrm{e}^{\widetilde{\mathcal{W}}_{0}(\tau) \sigma_{3}}\left(\begin{array}{cc}
0 & \frac{\mathrm{i} 2^{3 / 4} \alpha^{3 / 2}}{\sqrt{b(\tau)}} \\
\frac{\mathrm{i} \sqrt{b(\tau)}}{2^{3 / 4} \alpha^{3 / 2}} & 0
\end{array}\right) \\
& \times\left(\mathrm{I}+\mathcal{O}\left(\tau^{-2(\varepsilon-\delta)}\right)+\mathcal{O}\left(\tau^{-\frac{1}{3}+3 \varepsilon}\right)\right),
\end{aligned}
$$


where

$$
\begin{aligned}
\widetilde{\mathcal{W}}_{0}(\tau) \underset{\tau \rightarrow+\infty}{:=} \mathrm{i} 3 \sqrt{3} \alpha^{2} \tau^{2 / 3}+\mathrm{i} 2 \sqrt{3} \widetilde{\Lambda}^{2}+\mathcal{I}_{0}^{\sharp}(\tau)-C_{0}^{\mathrm{WKB}} \\
\quad-\frac{\mathrm{i}}{2 \sqrt{3}}\left(\left(a-\frac{\mathrm{i}}{2}\right)+\frac{h_{0}(\tau)}{\alpha^{2}}-\frac{\mathfrak{p}_{\tau}}{4}\right)\left(\frac{1}{3} \ln \tau-\ln \widetilde{\Lambda}\right) .
\end{aligned}
$$

Hence, via expansions (3.140) and (3.141), upon taking into account definition (3.91), and conditions (3.92) and (3.93), one arrives at

$$
L_{0}(\tau) \underset{\tau \rightarrow+\infty}{=}-\mathrm{i} \mathcal{R}_{2}^{-1} \exp \left(\left(\mathfrak{t}_{0}+\mathfrak{c}_{0}+\mathcal{I}_{0}^{\sharp}(\tau)\right) \sigma_{3}\right)\left(\sqrt{\frac{\varkappa_{0}(\tau) \hat{r}_{0}(\tau)}{\hat{u}_{0}(\tau)}}\right)^{\sigma_{3}} \sigma_{2}\left(\mathrm{I}+E_{0}(\tau)\right),
$$

where $\mathfrak{t}_{0}$ and $\mathfrak{c}_{0}$ are defined by Equations (3.138) and (3.139), respectively, and

$$
\begin{aligned}
E_{0}(\tau) \underset{\tau \rightarrow+\infty}{=} \tau^{6 \varepsilon+2 \varepsilon \operatorname{Re}(\nu+1)+\frac{\delta}{2}-\delta_{1}}\left(\begin{array}{cc}
\mathcal{O}(1) & \mathcal{O}\left(\varkappa_{0}(\tau) \tau^{-2 \varepsilon \operatorname{Re}(\nu+1)}\right) \\
\mathcal{O}\left(\left(\varkappa_{0}(\tau)\right)^{-1} \tau^{2 \varepsilon \operatorname{Re}(\nu+1)}\right) & \mathcal{O}(1)
\end{array}\right) \\
+\tau^{-2 \varepsilon}\left(\begin{array}{cc}
\mathcal{O}\left(\tau^{2 \delta}\right) & \mathcal{O}\left(\tau^{-2 \varepsilon \operatorname{Re}(\nu+1 / 2)}\right) \\
\mathcal{O}\left(\tau^{2 \delta+2 \varepsilon \operatorname{Re}(\nu+1 / 2)}\right) & \mathcal{O}\left(\tau^{\delta}\right)
\end{array}\right) \\
+\left(\mathcal{O}\left(\tau^{-2(\varepsilon-\delta)}\right)+\mathcal{O}\left(\tau^{-\frac{1}{3}+3 \varepsilon}\right)\right)\left(\begin{array}{cc}
\mathcal{O}(1) & o(1) \\
o(1) & \mathcal{O}(1)
\end{array}\right) .
\end{aligned}
$$

Invoking conditions (3.136), and conditions (3.92) and (3.93), one arrives at Equation (3.137).

Theorem 3.4.1. Assuming conditions (3.12), (3.92), (3.93), (3.128), (3.129), and (3.136), define $\delta^{0}:=\min \left\{\delta_{i j}^{0}\right\}_{i, j=1,2}$. Furthermore, suppose that

$$
\left(\frac{\tau^{\frac{1}{3} \operatorname{Im}(a)}\left(\hat{u}_{0}(\tau)\right)^{2}}{b(\tau)\left(\hat{r}_{0}(\tau)\right)^{2}}\right) \tau^{-\delta^{0}} \underset{\tau \rightarrow+\infty}{=} \mathcal{O}\left(\tau^{-\delta_{12}^{G}}\right), \quad\left(\frac{b(\tau)\left(\hat{r}_{0}(\tau)\right)^{2}}{\tau^{\frac{1}{3} \operatorname{Im}(a)}\left(\hat{u}_{0}(\tau)\right)^{2}}\right) \tau^{-\delta^{0}} \underset{\tau \rightarrow+\infty}{=} \mathcal{O}\left(\tau^{-\delta_{21}^{G}}\right),
$$

where $\delta_{12}^{G}, \delta_{21}^{G}>0$. Let

$$
\delta_{G}:=\min \left\{\delta^{0}, \delta_{12}^{G}, \delta_{21}^{G}, \delta_{12}^{\infty}, \delta_{21}^{\infty}\right\} .
$$

Then the connection matrix (cf. Equation (1.11) ) has the following asymptotics:

$$
G \underset{\tau \rightarrow+\infty}{=} G_{\infty}\left(\mathrm{I}+\mathcal{O}\left(\tau^{-\delta_{G}}\right)\right),
$$

where

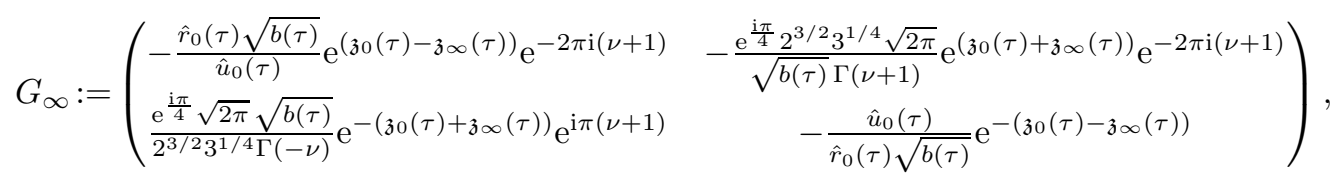

with

$$
\begin{gathered}
\mathfrak{z}_{\infty}(\tau)=\mathfrak{t}_{\infty}+\mathfrak{c}_{\infty}-\mathcal{I}_{\infty}^{\sharp}(\tau), \\
\mathfrak{z}_{0}(\tau)=\mathfrak{t}_{0}+\mathfrak{c}_{0}+\mathcal{I}_{0}^{\sharp}(\tau),
\end{gathered}
$$

where $\mathfrak{t}_{\infty}, \mathfrak{c}_{\infty}, \mathcal{I}_{\infty}^{\sharp}(\tau), \mathfrak{t}_{0}, \mathfrak{c}_{0}$, and $\mathcal{I}_{0}^{\sharp}(\tau)$ are defined by Equations (3.131), (3.132), (3.59), (3.138), (3.139), and (3.61), respectively.

Proof. Starting from the definition of the connection matrix (cf. Equation (1.11)), one has the following sequence of formulae: $Y_{0}^{\infty}(\mu):=X_{0}^{0}(\mu) G \Rightarrow$ (cf. Equations (3.5)) $Y_{0}^{\infty}\left(\tau^{-1 / 6} \widetilde{\mu}\right)=X_{0}^{0}\left(\tau^{-1 / 6} \widetilde{\mu}\right) G$ $\Rightarrow \tau^{-\frac{1}{12} \sigma_{3}} Y_{0}^{\infty}\left(\tau^{-1 / 6} \widetilde{\mu}\right)=\tau^{-\frac{1}{12} \sigma_{3}} X_{0}^{0}\left(\tau^{-1 / 6} \widetilde{\mu}\right) G \Rightarrow$ (cf. Lemmata 3.4.1 and 3.4.2) $\widetilde{\Psi}(\widetilde{\mu}, \tau) L_{\infty}(\tau)=$ $\widetilde{\Psi}(\widetilde{\mu}, \tau) L_{0}(\tau) G \Rightarrow L_{\infty}(\tau)=L_{0}(\tau) G \Rightarrow$

$$
G=\left(L_{0}(\tau)\right)^{-1} L_{\infty}(\tau) .
$$


Using Equations (3.130)-(3.132), Equations (3.137)-(3.139), and the formulae for $\mathcal{R}_{0}$ and $\mathcal{R}_{2}$ given in Remark 3.3.1, one arrives at

$$
G \underset{\tau \rightarrow+\infty}{=} G_{\infty}\left(\mathrm{I}+E_{G}(\tau)\right)
$$

where $G_{\infty}$ is defined by Equations (3.146) - (3.148), and

$$
E_{G}(\tau) \underset{\tau \rightarrow+\infty}{:=}\left(\begin{array}{cc}
\mathcal{O}\left(\tau^{-\delta_{11}^{\infty}}\right) & \mathcal{O}\left(\tau^{-\delta_{12}}\right) \\
\mathcal{O}\left(\tau^{-\delta_{21}}\right) & \mathcal{O}\left(\tau^{-\delta_{22}}\right)
\end{array}\right)+\left(G_{\infty}\right)^{-1}\left(\begin{array}{cc}
\mathcal{O}\left(\tau^{-\delta_{11}^{0}}\right) & \mathcal{O}\left(\tau^{-\delta_{12}^{0}}\right) \\
\mathcal{O}\left(\tau^{-\delta_{21}}\right) & \mathcal{O}\left(\tau^{-\delta_{22}}\right)
\end{array}\right) G_{\infty}
$$

The estimate for $E_{G}(\tau)$ (cf. Equations (3.144) and (3.145)) follows from the definitions of $\delta_{i j}^{\infty}$ and $\delta_{i j}^{0}$, $i, j=1,2$, given in Lemmata 3.4.1 and 3.4.2, respectively, and conditions (3.143).

\section{Asymptotic Solution of the Inverse Monodromy Problem}

In Section 3 the connection matrix, $G$, for Equation (3.6) is calculated asymptotically under certain conditions on its coefficient function, 13 . Using these conditions, one can derive the $\tau$-dependent class of functions to which $G$ belongs. We are not, however, going to proceed in this, most general, way; rather, we will invoke the isomonodromy condition on $G$, that is, its matrix elements, $g_{i j}, i, j=1,2$, are constants, and then invert the formula for $G$ to obtain the coefficient functions of Equation (3.6) and verify that they satisfy all of the imposed conditions for this isomonodromy case. The latter procedure produces explicit formulae for the coefficient functions of Equation (3.6), which give rise to asymptotics of the solution of the system of isomonodromy deformations (1.5) (see, also, Equations (3.5)) and, in turn, define asymptotics of the solution of the degenerate third Painlevé equation (1.1) and the related functions $\mathcal{H}(\tau)$ and $f(\tau)$ (cf. Equations (1.2) and (1.3), respectively). Zeroes and poles of the leading term of asymptotics of $u(\tau)$ define the centers of the cheese-holes of the domain $\widetilde{\mathcal{D}}_{u}$ (cf. definitions (2.10), (3.2), (3.3), and (3.4)). We also prove that, for large enough $\tau$, there is a one-to-one correspondence between the zeroes and poles of the solution $u(\tau)$ and the zeroes and poles of its leading term of asymptotics, that is, in each cheese-hole there is located one, and only one, zero or pole of $u(\tau)$.

Proposition 4.1. Let $g_{i j}, i, j=1,2$, denote the matrix elements of $G$. Assume that $g_{11} g_{12} g_{21} g_{22} \neq 0$ and the conditions of Theorem 3.4 .1 are valid. Then the functions $\hat{r}_{0}(\tau), \hat{u}_{0}(\tau), h_{0}(\tau), \varkappa_{0}^{2}(\tau)$, and $b(\tau)$ have the following asymptotics:

$$
\begin{gathered}
\hat{r}_{0}(\tau) \underset{\tau \rightarrow+\infty}{=} \frac{12}{1-\mathrm{i} \sqrt{2} \cos (\widetilde{\vartheta}(\tau))} \underset{\tau \rightarrow+\infty}{=} \frac{6 \cos \vartheta_{0}}{\cos \left(\frac{1}{2}\left(\widetilde{\vartheta}(\tau)+\vartheta_{0}\right)\right) \cos \left(\frac{1}{2}\left(\widetilde{\vartheta}(\tau)-\vartheta_{0}\right)\right)}, \\
\hat{u}_{0}(\tau) \underset{\tau \rightarrow+\infty}{=}-\frac{3}{2 \cos ^{2}\left(\frac{1}{2}\left(\widetilde{\vartheta}(\tau)+\vartheta_{0}\right)\right)}+\mathcal{O}\left(\tau^{-\frac{1}{3}+\delta-\delta_{1}}\right) \underset{\tau \rightarrow+\infty}{=}-\frac{\sin ^{2} \vartheta_{0}}{\cos ^{2}\left(\frac{1}{2}\left(\widetilde{\vartheta}(\tau)+\vartheta_{0}\right)\right)}+\mathcal{O}\left(\tau^{-\frac{1}{3}+\delta-\delta_{1}}\right), \\
h_{0}(\tau) \underset{\tau \rightarrow+\infty}{=} \mathrm{i} 2 \sqrt{3} \alpha^{2}\left((\widetilde{\nu}+1)-\frac{1}{2}+\frac{\mathrm{i}}{2 \sqrt{3}}\left(a-\frac{\mathrm{i}}{2}\right)-\frac{\sin (\widetilde{\vartheta}(\tau)) \cos \vartheta_{0}}{2 \sqrt{2} \cos \left(\frac{1}{2}\left(\widetilde{\vartheta}(\tau)+\vartheta_{0}\right)\right) \cos \left(\frac{1}{2}\left(\widetilde{\vartheta}(\tau)-\vartheta_{0}\right)\right)}\right. \\
\left.\quad+\mathcal{O}\left(\tau^{-\delta_{G}}\right)+\mathcal{O}\left(\tau^{\delta-2 \delta_{1}}\right)\right), \\
\varkappa_{0}^{2}(\tau) \underset{\tau \rightarrow+\infty}{=} \mathrm{i} 8 \sqrt{3}\left((\widetilde{\nu}+1)-\frac{1}{2}+\frac{\mathrm{i}}{2 \sqrt{3}}\left(a-\frac{\mathrm{i}}{2}\right)-\frac{\sin (\widetilde{\vartheta}(\tau)) \cos \vartheta_{0}}{2 \sqrt{2} \cos \left(\frac{1}{2}\left(\widetilde{\vartheta}(\tau)+\vartheta_{0}\right)\right) \cos \left(\frac{1}{2}\left(\widetilde{\vartheta}(\tau)-\vartheta_{0}\right)\right)}\right) \\
+\frac{4\left(a-\frac{\mathrm{i}}{2}\right) \cos { }^{2}\left(\frac{1}{2}\left(\widetilde{\vartheta}(\tau)+\vartheta_{0}\right)\right)}{\cos \left(\frac{1}{2}\left(\widetilde{\vartheta}(\tau)+\vartheta_{0}\right)+\vartheta_{0}\right) \cos \left(\frac{1}{2}\left(\widetilde{\vartheta}(\tau)+\vartheta_{0}\right)-\vartheta_{0}\right)}+\mathcal{O}\left(\tau^{-\delta_{G}}\right)+\mathcal{O}\left(\tau^{\delta-2 \delta_{1}}\right)+\mathcal{O}\left(\tau^{-\frac{2}{3}+3 \delta}\right), \\
\sqrt{b(\tau)} \underset{\tau \rightarrow+\infty}{=}\left(\frac{\cos \left(\frac{1}{2}\left(\widetilde{\vartheta}(\tau)-\vartheta_{0}\right)\right)}{4 \cos \left(\vartheta_{0}\right) \cos \left(\frac{1}{2}\left(\widetilde{\vartheta}(\tau)+\vartheta_{0}\right)\right)}+\mathcal{O}\left(\tau^{-\frac{2}{3}}\right)+\mathcal{O}\left(\tau^{\delta-2 \delta_{1}}\right)\right) \exp (\Phi(\tau)),
\end{gathered}
$$

${ }^{13}$ See Equations (3.7)-(3.11) and (3.13), and conditions (3.12), 3.92), (3.93), 3.128), (3.129), (3.136), and (3.143). 
where

$$
\begin{gathered}
\widetilde{\nu}+1:=\frac{\mathrm{i}}{2 \pi} \ln \left(g_{11} g_{22}\right), \quad 0<\operatorname{Re}(\widetilde{\nu}+1)<1 \\
\widetilde{\vartheta}(\tau):=6 \sqrt{3} \alpha^{2} \tau^{2 / 3}-\mathrm{i}\left((\widetilde{\nu}+1)-\frac{1}{2}\right) \ln \left(6 \sqrt{3} \alpha^{2} \tau^{2 / 3}\right)-\mathrm{i}\left((\widetilde{\nu}+1)-\frac{1}{2}\right) \ln 12+\left(a-\frac{\mathrm{i}}{2}\right) \ln (2+\sqrt{3}) \\
+\mathrm{i} \ln \left(\frac{g_{11} g_{12} \Gamma(\widetilde{\nu}+1)}{\sqrt{2 \pi}}\right)-\frac{3 \pi}{2}(\widetilde{\nu}+1)+\frac{7 \pi}{4}+\mathcal{O}\left(\tau^{-\delta_{G}} \ln \tau\right) \\
\vartheta_{0}:=-\frac{\pi}{2}+\frac{\mathrm{i}}{2} \ln (2+\sqrt{3}), \quad \cos \vartheta_{0}=\frac{\mathrm{i}}{\sqrt{2}}, \quad \sin \vartheta_{0}=-\sqrt{\frac{3}{2}} \\
\Phi(\tau):=-\mathrm{i} 3 \alpha^{2} \tau^{2 / 3}-\frac{\mathrm{i} a}{6} \ln \tau+\mathrm{i} \pi\left((\widetilde{\nu}+1)-\frac{1}{2}\right)+\ln g_{11}+2 \ln (2 \alpha)+\frac{\mathrm{i} a}{2} \ln \left(\frac{\alpha^{2}}{2}\right) \\
-\left((\widetilde{\nu}+1)-\frac{1}{2}\right) \ln (2+\sqrt{3})+\mathcal{O}\left(\tau^{-\delta_{G}}\right)
\end{gathered}
$$

and $\delta_{G}>0$ is defined by Equation (3.144).

Proof. Multiplying the diagonal elements of $G$ (cf. Equations (3.145)-(3.148) and taking into account conditions (3.92) and definition (4.6), one shows that

$$
\nu+1 \underset{\tau \rightarrow+\infty}{=}(\widetilde{\nu}+1)\left(1+\mathcal{O}\left(\tau^{-\delta_{G}}\right)\right),
$$

where $\delta_{G}>0$ is defined by Equation (3.144).

Multiplying the elements of the first row of $G$, one shows, via Equation (4.10), that

$$
g_{11} g_{12} \underset{\tau \rightarrow+\infty}{=} \exp \left(\frac{\mathrm{i} \pi}{4}+\frac{1}{2} \ln 2 \pi+\ln \left(2^{\frac{3}{2}} 3^{\frac{1}{4}}\right)-\ln \Gamma(\widetilde{\nu}+1)-4 \pi \mathrm{i}(\widetilde{\nu}+1)+\ln \left(\frac{\hat{r}_{0}(\tau)}{\hat{u}_{0}(\tau)}\right)+2 \mathfrak{z}_{0}(\tau)+\mathcal{O}\left(\tau^{-\delta_{G}}\right)\right),
$$

where, via Equations (3.148), (3.139), (3.61), and (4.10),

$$
\begin{aligned}
2 \mathfrak{z}_{0}(\tau) \underset{\tau \rightarrow+\infty}{=} & 2 \mathfrak{t}_{0}-3 \ln 2-\frac{1}{2} \ln 3+\frac{3 \pi \mathrm{i}}{2}+2(\widetilde{\nu}+1) \ln \left(\mathrm{e}^{\frac{\mathrm{i} \pi}{4}} 2^{\frac{3}{2}} 3^{\frac{1}{4}}\right)+2 \mathrm{i}\left(a-\frac{\mathrm{i}}{2}\right) \ln \left(\frac{\sqrt{3}+1}{\sqrt{2}}\right) \\
& +2\left((\widetilde{\nu}+1)-\frac{1}{2}\right) \ln 3 \alpha+2 \pi \mathrm{i}(\widetilde{\nu}+1)-\ln \left(\frac{\hat{r}_{0}(\tau)}{\hat{u}_{0}(\tau)}\right)-\frac{1}{2} \ln \mathfrak{Q}(\tau)+\mathcal{O}\left(\tau^{-\delta_{G}}\right),
\end{aligned}
$$

with $\mathfrak{t}_{0}$ defined by Equation (3.138), and

$$
\mathfrak{Q}(\tau):=\left(\frac{\hat{r}_{0}(\tau)-4 \sqrt{3}+\sqrt{32+\left(\hat{r}_{0}(\tau)-4\right)^{2}}}{\hat{r}_{0}(\tau)-4 \sqrt{3}-\sqrt{32+\left(\hat{r}_{0}(\tau)-4\right)^{2}}}\right)\left(\frac{\hat{r}_{0}(\tau)+4 \sqrt{3}-\sqrt{32+\left(\hat{r}_{0}(\tau)-4\right)^{2}}}{\hat{r}_{0}(\tau)+4 \sqrt{3}+\sqrt{32+\left(\hat{r}_{0}(\tau)-4\right)^{2}}}\right) .
$$

Substituting Equations (4.12) and (4.13) into Equation (4.11) and solving for $\mathfrak{Q}(\tau)$, one arrives at

$$
\frac{12-\hat{r}_{0}(\tau)-\sqrt{3} \sqrt{32+\left(\hat{r}_{0}(\tau)-4\right)^{2}}}{12-\hat{r}_{0}(\tau)+\sqrt{3} \sqrt{32+\left(\hat{r}_{0}(\tau)-4\right)^{2}}} \underset{\tau \rightarrow+\infty}{=} \exp (2 \mathrm{i} \widetilde{\vartheta}(\tau)),
$$

where $\widetilde{\vartheta}(\tau)$ is defined by Equation (4.7). There are two solutions of Equation (4.14) for $\hat{r}_{0}(\tau)$. Define the parameter $\vartheta_{0}$ by the first equation of (4.8); then, one of the solutions for $\hat{r}_{0}(\tau)$ is given in Equation (4.1), whilst the other is given by the same formula as in Equation (4.1) but with the change $\widetilde{\vartheta}(\tau) \rightarrow \widetilde{\vartheta}(\tau)+\pi$. This ambiguity in the solution of the inverse monodromy problem can be rectified by calculating one additional parameter, namely, one of the Stokes multiplier:14; however, we choose a different approach. There is a common domain of validity of the results obtained in this paper and

\footnotetext{
${ }^{14}$ This ambiguity is distinct from the one for the direct monodromy problem mentioned in Remark 1.3
} 
Part I [1]. In Appendix B, this fact is used in order to justify the choice for $\hat{r}_{0}(\tau)$ that is given in Equation (4.1). Furthermore, as a by-product of Equations (4.14), (4.8), and (4.1), one shows that

$$
\sqrt{32+\left(\hat{r}_{0}(\tau)-4\right)^{2}} \underset{\tau \rightarrow+\infty}{=}-\frac{4 \sqrt{6} \sin \widetilde{\vartheta}(\tau)}{1-\mathrm{i} \sqrt{2} \cos \widetilde{\vartheta}(\tau)} \underset{\tau \rightarrow+\infty}{=} \frac{2 \sin (\widetilde{\vartheta}(\tau)) \sin 2 \vartheta_{0}}{\cos \left(\frac{1}{2}\left(\widetilde{\vartheta}(\tau)+\vartheta_{0}\right)\right) \cos \left(\frac{1}{2}\left(\widetilde{\vartheta}(\tau)-\vartheta_{0}\right)\right)} .
$$

Via Equations (3.77), (4.1), (4.8), and (4.15), and conditions (3.12), one shows that

$$
\hat{u}_{0}(\tau) \underset{\tau \rightarrow+\infty}{=}-\frac{3}{2} \frac{\left(\mathrm{i} \sqrt{2}\left(\cos \left(\vartheta_{0}\right)+\cos \widetilde{\vartheta}(\tau)\right)-\sqrt{6}\left(\sin \left(\vartheta_{0}\right)+\sin \widetilde{\vartheta}(\tau)\right)\right)}{\left(\cos \left(\vartheta_{0}\right)+\cos \widetilde{\vartheta}(\tau)\right)^{2}}+\mathcal{O}\left(\tau^{-\frac{1}{3}+\delta-\delta_{1}}\right) .
$$

Now, from Equations (4.8), (4.10), and (4.16), and the trigonometric identities $\sin \left(\vartheta_{0}\right)+\sin \widetilde{\vartheta}(\tau)=$ $2 \sin \left(\frac{1}{2}\left(\vartheta_{0}+\widetilde{\vartheta}(\tau)\right)\right) \cos \left(\frac{1}{2}\left(\vartheta_{0}-\widetilde{\vartheta}(\tau)\right)\right)$ and $\cos \left(\vartheta_{0}\right)+\cos \widetilde{\vartheta}(\tau)=2 \cos \left(\frac{1}{2}\left(\vartheta_{0}+\widetilde{\vartheta}(\tau)\right)\right) \cos \left(\frac{1}{2}\left(\vartheta_{0}-\widetilde{\vartheta}(\tau)\right)\right)$, one arrives at Equation (4.2). To obtain $h_{0}(\tau)$ (cf. Equation (4.3)), one uses Equations (3.68), (3.91), (4.8), (4.10), and (4.15), and conditions (3.12). Finally, using Equations (3.65), (4.2), and (4.3), one arrives at Equation (4.4) for $\varkappa_{0}^{2}(\tau)$.

It follows from the (12)-element of $G$ and Equation (4.10) that

$$
\sqrt{b(\tau)} \underset{\tau \rightarrow+\infty}{=} \exp \left(\frac{5 \pi \mathrm{i}}{4}+\ln \left(2^{\frac{3}{2}} 3^{\frac{1}{4}}\right)-2 \pi \mathrm{i}(\widetilde{\nu}+1)+\ln \left(\frac{\sqrt{2 \pi}}{g_{12} \Gamma(\widetilde{\nu}+1)}\right)+\mathfrak{z}_{0}(\tau)+\mathfrak{z}_{\infty}(\tau)+\mathcal{O}\left(\tau^{-\delta_{G}}\right)\right),
$$

where $\mathfrak{z}_{0}(\tau)$ is given in Equations (4.12) and (4.13), and (cf. Equation (3.147)) $\mathfrak{z}_{\infty}(\tau)=\mathfrak{t}_{\infty}+\mathfrak{c}_{\infty}-\mathcal{I}_{\infty}^{\sharp}(\tau)$, with $\mathfrak{t}_{\infty}, \mathfrak{c}_{\infty}$, and $\mathcal{I}_{\infty}^{\sharp}(\tau)$ defined, respectively, by Equations (3.131), (3.132), and (3.59). Hence, using Equations (4.14) and (4.17), one shows that

$$
\sqrt{b(\tau)} \underset{\tau \rightarrow+\infty}{=} \frac{\hat{u}_{0}(\tau)}{\hat{r}_{0}(\tau)} \exp (\Phi(\tau))
$$

where $\Phi(\tau)$ is defined by Equation (4.9), whence, via Equations (4.1) and (4.2), and conditions (3.12), one arrives at Equation (4.5).

Remark 4.1. It is important to note that, as a consequence of Equation (4.18),

$$
\frac{\tau^{\frac{1}{6} \operatorname{Im}(a)} \hat{u}_{0}(\tau)}{\sqrt{b(\tau)} \hat{r}_{0}(\tau)} \underset{\tau \rightarrow+\infty}{=} \mathcal{O}(1)
$$

therefore, conditions (3.143) are satisfied; moreover, via conditions (3.136) and the estimate (4.19), it follows that conditions (3.128) and (3.129) are also satisfied. Making the following choice for the parameters $\varepsilon, \delta$, and $\delta_{1}$,

$$
0<20 \delta<10 \varepsilon<\delta_{1}<1 / 3,
$$

one shows that conditions (3.93) and (3.136) are valid uniformly for $0<\operatorname{Re}(\widetilde{\nu}+1)<1$. For the functions $\hat{r}_{0}(\tau), \hat{u}_{0}(\tau)$, and $h_{0}(\tau)$ defined, respectively, by Equations (4.1), (4.2), and (4.3), conditions (3.12) are satisfied.

Proposition 4.2. Under the conditions of Proposition 4.1, the functions $a(\tau), b(\tau), c(\tau)$, and $d(\tau)$, defining, via Equations (3.5), the solution of the system of isomonodromy deformations (1.5), have the following asymptotic representations:

$$
\begin{gathered}
\sqrt{-a(\tau) b(\tau)} \underset{\tau \rightarrow+\infty}{=} \frac{2 \alpha^{4} \cos \left(\kappa(\tau)+\vartheta_{0}\right) \cos \left(\kappa(\tau)-\vartheta_{0}\right)}{\cos ^{2}(\kappa(\tau))}+\mathcal{O}\left(\tau^{-\frac{1}{3}+\delta-\delta_{1}}\right), \\
a(\tau) d(\tau) \underset{\tau \rightarrow+\infty}{=} \frac{\mathrm{i} 4 \alpha^{6} \cos \left(\vartheta_{0}\right) \cos \left(\kappa(\tau)+\vartheta_{0}\right) \cos \left(\kappa(\tau)-\vartheta_{0}\right)}{\cos ^{2}(\kappa(\tau))}\left(\cos \vartheta_{0}+\frac{\sin ^{2} \vartheta_{0}}{\cos (\kappa(\tau)) \cos \left(\kappa(\tau)-\vartheta_{0}\right)}\right) \\
\quad+\mathcal{O}\left(\tau^{-\frac{2}{3}+2 \delta}\right)+\mathcal{O}\left(\tau^{-\frac{1}{3}+\delta-\delta_{1}}\right), \\
b(\tau) c(\tau) \underset{\tau \rightarrow+\infty}{=}-\frac{\mathrm{i} 4 \alpha^{6} \cos \left(\kappa(\tau)+\vartheta_{0}\right) \cos \left(\kappa(\tau)-\vartheta_{0}\right)}{\cos ^{2}(\kappa(\tau))}\left(\frac{\cos \left(\vartheta_{0}\right) \sin ^{2} \vartheta_{0}}{\cos (\kappa(\tau)) \cos \left(\kappa(\tau)-\vartheta_{0}\right)}\right.
\end{gathered}
$$




$$
\begin{aligned}
&+\left.\frac{\sin ^{2} \vartheta_{0}}{\cos \left(\kappa(\tau)+\vartheta_{0}\right) \cos \left(\kappa(\tau)-\vartheta_{0}\right)}-\cos ^{2} \vartheta_{0}\right)+\mathcal{O}\left(\tau^{-\frac{2}{3}+2 \delta}\right)+\mathcal{O}\left(\tau^{-\frac{1}{3}+\delta-\delta_{1}}\right), \\
& c(\tau) d(\tau) \underset{\tau \rightarrow+\infty}{=}-4 \alpha^{4}\left(\cos ^{2} \vartheta_{0}+\frac{\cos \vartheta_{0}}{\cos (\kappa(\tau)) \cos \left(\kappa(\tau)-\vartheta_{0}\right)}\right)\left(\frac{\cos \left(\vartheta_{0}\right) \sin ^{2} \vartheta_{0}}{\cos (\kappa(\tau)) \cos \left(\kappa(\tau)-\vartheta_{0}\right)}\right. \\
&\left.\quad+\frac{\sin ^{2} \vartheta_{0}}{\cos \left(\kappa(\tau)+\vartheta_{0}\right) \cos \left(\kappa(\tau)-\vartheta_{0}\right)}-\cos ^{2} \vartheta_{0}\right)+\mathcal{O}\left(\tau^{-\frac{1}{3}+\delta-\delta_{1}}\right),
\end{aligned}
$$

where $b(\tau)$ is given in Equation (4.5) (see, also, Equation (4.9) $), \kappa(\tau):=\left(\widetilde{\vartheta}(\tau)+\vartheta_{0}\right) / 2$, and $\widetilde{\vartheta}(\tau), \vartheta_{0}$, and $\delta_{G}>0$ are defined, respectively, by Equations (4.7), (4.8), and (3.144).

Proof. If $g_{i j}, i, j=1,2$, are $\tau$-dependent, then any functions whose asymptotics are given by Equations (4.1)-(4.5) satisfy conditions (3.12), (3.92), (3.93), (3.128), (3.129), (3.136), and (3.143); therefore, one can now use the justification scheme suggested in [10] (see, also, [14). Equations (4.20)(4.23) are obtained from Equations (3.11), (3.14), (3.15), and (3.17) via the direct substitution of Equations (4.1) and (4.2).

From Proposition 1.2, Equations (3.5), and Equation (3.11), one shows that $u(\tau)$, the solution of the degenerate third Painlevé equation (1.1), is given by

$$
u(\tau)=\epsilon \tau^{1 / 3} \sqrt{-a(\tau) b(\tau)}=\frac{\epsilon(\epsilon b)^{2 / 3}}{2} \tau^{1 / 3}\left(1+\hat{u}_{0}(\tau)\right), \quad \epsilon= \pm 1
$$

It was shown in [1] that, in terms of the function $h_{0}(\tau)$, the Hamiltonian (cf. Equation (1.2) $), \mathcal{H}(\tau)$, corresponding to $u(\tau)$, is given by

$$
\mathcal{H}(\tau)=3(\epsilon b)^{2 / 3} \tau^{1 / 3}-4 h_{0}(\tau) \tau^{-1 / 3}+\frac{1}{2 \tau}\left(a-\frac{\mathrm{i}}{2}\right)^{2}
$$

Finally, the function $f(\tau)$ (cf. Equation (1.3) ) can be presented in terms of $\hat{r}_{0}(\tau)$ with the help of Equations (3.10), (3.8), (3.5), and (1.7):

$$
f(\tau)=-\frac{\mathrm{i}(\epsilon b)^{1 / 3}}{2} \tau^{2 / 3}\left(1-\frac{\hat{r}_{0}(\tau)}{2}\right)-\frac{1}{2}\left(\mathrm{i} a+\frac{1}{2}\right) .
$$

Thus, Proposition 4.1 implies the corresponding asymptotic results for the functions $\hat{r}_{0}(\tau), \mathcal{H}(\tau)$, and $f(\tau)$ stated in Theorem 2.1.

It is important to note that asymptotics of $\hat{r}_{0}(\tau)$ can also be reformulated as asymptotics of the logarithmic derivative of $u(\tau)$.

Proposition 4.3. The leading term of asymptotics of $(\ln u(\tau))^{\prime}$ coincides with the logarithmic derivative of the leading term of asymptotics of the function $u(\tau)$ given in Theorem 2.1, Equation (2.4):

$$
\frac{\mathrm{d} \ln u(\tau)}{\mathrm{d} \tau} \underset{\tau \rightarrow \infty \mathrm{e}^{\mathrm{i} \pi \varepsilon_{1}}}{=} \frac{3 \sqrt{3}(-1)^{\varepsilon_{2}}(\epsilon b)^{1 / 3} \tau^{-1 / 3} \cos \left(\frac{\vartheta}{2}\right)}{\sin \left(\frac{\vartheta}{2}\right) \sin \left(\frac{\vartheta}{2}-\vartheta_{0}\right) \sin \left(\frac{\vartheta}{2}+\vartheta_{0}\right)}+\mathcal{O}\left(\tau^{-\frac{2}{3}+\delta-\delta_{1}}\right),
$$

where $\vartheta=\vartheta\left(\varepsilon_{1}, \varepsilon_{2}, \tau\right)$ is defined by Equations (2.2), (2.5), and (2.6), and $\vartheta_{0}$ is given in Equation (2.7).

Proof. As in the main body of the paper, the case $\varepsilon_{1}=\varepsilon_{2}=0$ is considered in detail; in particular,

$$
\vartheta=\vartheta(0,0, \tau)=\widetilde{\vartheta}(\tau)+\vartheta_{0}-\pi:
$$

the remaining cases are proved analogously upon applying the transformation $\mathcal{F}_{\varepsilon_{1}, \varepsilon_{2}}$ introduced at the beginning of Section 2, From Equations (4.26) and (1.3), one shows that

$$
\frac{u^{\prime}(\tau)}{u(\tau)}=\frac{\mathrm{i} b}{u(\tau)}-\frac{2 \mathrm{i}(\epsilon b)^{1 / 3}}{\tau^{1 / 3}}\left(1-\frac{\hat{r}_{0}(\tau)}{2}\right) .
$$

Substituting into Equation (4.28) the asymptotics for $u(\tau)$ and $\hat{r}_{0}(\tau)$ given in Equations (2.4) and (4.1), respectively, and using the identity

$$
2 \sin \left(\frac{\vartheta}{2}\right) \sin \left(\frac{\vartheta}{2}-\vartheta_{0}\right)=\cos \vartheta_{0}-\cos \left(\vartheta-\vartheta_{0}\right)
$$


one arrives at

$$
\frac{u^{\prime}(\tau)}{u(\tau)} \underset{\tau \rightarrow+\infty}{=} \frac{\mathrm{i}(\epsilon b)^{1 / 3}\left(2 \sin ^{3}\left(\frac{\vartheta}{2}\right)+5 \cos \left(\vartheta_{0}\right) \sin \left(\frac{\vartheta}{2}+\vartheta_{0}\right)+\sin \left(\frac{\vartheta}{2}+\vartheta_{0}\right) \cos \left(\vartheta-\vartheta_{0}\right)\right)}{\tau^{1 / 3} \sin \left(\frac{\vartheta}{2}\right) \sin \left(\frac{\vartheta}{2}-\vartheta_{0}\right) \sin \left(\frac{\vartheta}{2}+\vartheta_{0}\right)}+\mathcal{O}\left(\tau^{-\frac{2}{3}+\delta-\delta_{1}}\right) .
$$

Consider, now, the numerator of the above fraction. One uses the identities

$$
\begin{aligned}
\sin \left(\frac{\vartheta}{2}+\vartheta_{0}\right) & =\sin \left(\frac{\vartheta}{2}\right) \cos \vartheta_{0}+\cos \left(\frac{\vartheta}{2}\right) \sin \vartheta_{0}, \\
\sin \left(\frac{\vartheta}{2}+\vartheta_{0}\right) \cos \left(\vartheta-\vartheta_{0}\right) & =\frac{1}{2} \sin \left(\frac{3 \vartheta}{2}\right)-\frac{1}{2} \sin \left(\frac{\vartheta}{2}-2 \vartheta_{0}\right) \\
& =\frac{1}{2} \sin \left(\frac{3 \vartheta}{2}\right)-\frac{1}{2}\left(2 \cos ^{2} \vartheta_{0}-1\right) \sin \left(\frac{\vartheta}{2}\right)+\sin \left(\vartheta_{0}\right) \cos \left(\vartheta_{0}\right) \cos \left(\frac{\vartheta}{2}\right),
\end{aligned}
$$

to transform the second and third terms, respectively, of the summand: regrouping, now, the terms in the numerator, one transforms it into the form

$$
\frac{1}{2}\left(4 \sin ^{3}\left(\frac{\vartheta}{2}\right)+\sin \left(\frac{3 \vartheta}{2}\right)+\left(8 \cos ^{2} \vartheta_{0}+1\right) \sin \left(\frac{\vartheta}{2}\right)\right)+6 \sin \left(\vartheta_{0}\right) \cos \left(\vartheta_{0}\right) \cos \left(\frac{\vartheta}{2}\right)=-\mathrm{i} 3 \sqrt{3} \cos \left(\frac{\vartheta}{2}\right),
$$

where, in order to get the last equality, one uses the values of $\sin \vartheta_{0}$ and $\cos \vartheta_{0}$ given in Equation (4.8). Thus, asymptotics (4.27) is proved.

To finish the proof, one has to confirm that the logarithmic derivative of the leading term of asymptotics (2.4) coincides, modulo $\mathcal{O}\left(\tau^{-\frac{2}{3}+\delta-\delta_{1}}\right)$, with the leading term of asymptotics (4.27). Taking the logarithmic derivative of asymptotics (2.4), neglecting terms that are $\mathcal{O}\left(\tau^{-1}\right)$, and assuming that the derivatives of the errors can also be neglected, one shows that

$$
\begin{aligned}
& \frac{\sqrt{3}(\epsilon b)^{1 / 3}}{\tau^{1 / 3}}\left(\frac{\cos \left(\frac{\vartheta}{2}-\vartheta_{0}\right)}{\sin \left(\frac{\vartheta}{2}-\vartheta_{0}\right)}+\frac{\cos \left(\frac{\vartheta}{2}+\vartheta_{0}\right)}{\sin \left(\frac{\vartheta}{2}+\vartheta_{0}\right)}-\frac{2 \cos \left(\frac{\vartheta}{2}\right)}{\sin \left(\frac{\vartheta}{2}\right)}\right) \\
& =\frac{\sqrt{3}(\epsilon b)^{1 / 3} \cos \left(\frac{\vartheta}{2}\right)}{\tau^{1 / 3}}\left(\frac{2 \sin \left(\frac{\vartheta}{2}\right)}{\sin \left(\frac{\vartheta}{2}-\vartheta_{0}\right) \sin \left(\frac{\vartheta}{2}+\vartheta_{0}\right)}-\frac{2}{\sin \left(\frac{\vartheta}{2}\right)}\right) \\
& =\frac{\sqrt{3}(\epsilon b)^{1 / 3} \cos \left(\frac{\vartheta}{2}\right)\left(2 \sin ^{2}\left(\frac{\vartheta}{2}\right)+\cos (\vartheta)-\cos \left(2 \vartheta_{0}\right)\right)}{\tau^{1 / 3} \sin \left(\frac{\vartheta}{2}\right) \sin \left(\frac{\vartheta}{2}-\vartheta_{0}\right) \sin \left(\frac{\vartheta}{2}+\vartheta_{0}\right)} .
\end{aligned}
$$

One completes the proof upon noting that $2 \sin ^{2}(\vartheta / 2)+\cos (\vartheta)-\cos \left(2 \vartheta_{0}\right)=2 \sin ^{2} \vartheta_{0}=3$.

Consider, now, more carefully, the cheese-like domain $\widetilde{\mathcal{D}}_{u}$ (cf. Equation (3.4)). As follows from the leading term of asymptotics (2.4) for the case $\operatorname{Re}(\widetilde{\nu}+1) \neq 1 / 2$, where $\widetilde{\nu}:=\widetilde{\nu}(0,0)$, the countable sets $\mathcal{P}_{u}^{\delta}$ and $\mathcal{Z}_{u}^{\delta}$ are empty, that is, $\widetilde{\mathcal{D}}_{u}$ coincides with the strip $\mathcal{D}$ (cf. Equation (2.10). In this case, $\delta$ is vacuous: strictly speaking, one can improve some of the error estimates in Section 3 and this section by assuming, in lieu of conditions (3.12), the following conditions for the coefficients:

$$
\hat{r}_{0}(\tau) \underset{\tau \rightarrow+\infty}{\sim} \mathcal{O}\left(\tau^{-\frac{1}{3}+\delta}\right), \quad \hat{u}_{0}(\tau) \underset{\tau \rightarrow+\infty}{\sim} \mathcal{O}\left(\tau^{-\frac{1}{3}+\delta}\right), \quad \hat{h}_{0}(\tau) \underset{\tau \rightarrow+\infty}{\sim} \mathcal{O}(1) .
$$

To eschew this, we can formally substitute $\delta=0$ in our calculations; surely, though, we will not achieve the best possible error estimates, but they are sufficient for our purposes. Consider the definition of $\delta_{G}$ given in Equation (3.144), where $\delta_{12}^{G}=\delta_{21}^{G}=\delta^{0}$ (cf. Equations (3.143) and (4.19)). Put $\delta=0$ in the formulae for $\delta_{12}^{\infty}$ and $\delta_{21}^{\infty}$, and $\delta^{0}$ defined, respectively, in Lemma 3.4.1, and Theorem 3.4.1] and Lemma 3.4.2. Analyzing the resulting system for $\delta_{G}$, one has to choose $\varepsilon \in(0,1 / 9)$ so that $\delta_{G}$ attains its maximum; the result reads:

(i) for $\operatorname{Re}(\widetilde{\nu}+1) \in(0,1 / 2)$ and $\varepsilon=\delta_{1} /(7+6 \operatorname{Re}(\widetilde{\nu}+1))$,

$$
0<\delta_{G}<\delta_{1}\left(\frac{1+2 \operatorname{Re}(\widetilde{\nu}+1)}{7+6 \operatorname{Re}(\widetilde{\nu}+1)}\right)
$$

(ii) for $\operatorname{Re}(\widetilde{\nu}+1) \in(1 / 2,1)$ and $\varepsilon=\delta_{1} /(9+2 \operatorname{Re}(\widetilde{\nu}+1))$,

$$
0<\delta_{G}<\delta_{1}\left(\frac{3-2 \operatorname{Re}(\widetilde{\nu}+1)}{9+2 \operatorname{Re}(\widetilde{\nu}+1)}\right) .
$$


Together with the justification scheme of [10, this completes the proof of Theorem 2.1.

The structure of the cheese-like domain $\widetilde{\mathcal{D}}_{u}$ for $\operatorname{Re}(\widetilde{\nu}+1)=1 / 2$ is more complicated: to study this case, it is convenient to introduce the following definition.

Definition 4.1. Define $\hat{\vartheta}$ as the leading term of asymptotics of $\vartheta$ (cf. Equation (2.5)), that is,

$$
\vartheta=\hat{\vartheta}+\mathcal{O}\left(\tau^{-\delta_{G}} \ln \tau\right)
$$

Proposition 4.4. Suppose that

$$
g_{11}\left(\varepsilon_{1}, \varepsilon_{2}\right) g_{12}\left(\varepsilon_{1}, \varepsilon_{2}\right) g_{21}\left(\varepsilon_{1}, \varepsilon_{2}\right) g_{22}\left(\varepsilon_{1}, \varepsilon_{2}\right) \neq 0, \quad \operatorname{Re}\left(\frac{\mathrm{i}}{2 \pi} \ln \left(g_{11}\left(\varepsilon_{1}, \varepsilon_{2}\right) g_{22}\left(\varepsilon_{1}, \varepsilon_{2}\right)\right)\right)=\frac{1}{2},
$$

and the branch of the function $\ln (\cdot)$ is chosen so that $\operatorname{Im}\left(\ln \left(-g_{11}\left(\varepsilon_{1}, \varepsilon_{2}\right) g_{22}\left(\varepsilon_{1}, \varepsilon_{2}\right)\right)\right)=0$. For $m \in \mathbb{N}$, consider the following transcendental equations:

$$
\frac{\hat{\vartheta}}{2}=\pi m, \quad \frac{\hat{\vartheta}}{2}+\vartheta_{0}=\pi m, \quad \frac{\hat{\vartheta}}{2}-\vartheta_{0}=\pi m .
$$

There exist unique solutions of these equations, $\tau_{a s, m}^{\infty}, \tau_{a s, m}^{+}$, and $\tau_{a s, m}^{-}$, respectively 15 , the signs of the real parts of which correspond to $(-1)^{\varepsilon_{1}}$; furthermore,

$$
\begin{aligned}
& \tau_{a s, m}^{\infty} \underset{m \rightarrow \infty}{=} \mathrm{e}^{\mathrm{i} \pi \varepsilon_{1}}\left(\frac{2 \pi(-1)^{\varepsilon_{2}} m}{3 \sqrt{3}(\epsilon b)^{1 / 3}}\right)^{3 / 2}\left(1-\frac{3 \varrho_{1}\left(\varepsilon_{1}, \varepsilon_{2}\right)}{4 \pi} \frac{\ln m}{m}-\frac{3 \varrho_{2}\left(\varepsilon_{1}, \varepsilon_{2}\right)}{4 \pi} \frac{1}{m}+\mathcal{O}\left(\frac{\ln ^{2} m}{m^{2}}\right)\right), \\
& \tau_{a s, m}^{ \pm} \underset{m \rightarrow \infty}{=} \mathrm{e}^{\mathrm{i} \pi \varepsilon_{1}}\left(\frac{2 \pi(-1)^{\varepsilon_{2}} m}{3 \sqrt{3}(\epsilon b)^{1 / 3}}\right)^{3 / 2}\left(1-\frac{3 \varrho_{1}\left(\varepsilon_{1}, \varepsilon_{2}\right)}{4 \pi} \frac{\ln m}{m}-\frac{3}{4 \pi}\left(\varrho_{2}\left(\varepsilon_{1}, \varepsilon_{2}\right) \pm 2 \vartheta_{0}\right) \frac{1}{m}+\mathcal{O}\left(\frac{\ln ^{2} m}{m^{2}}\right)\right),
\end{aligned}
$$

where

$$
\begin{gathered}
\varrho_{1}\left(\varepsilon_{1}, \varepsilon_{2}\right):=\frac{1}{2 \pi} \ln \left(-g_{11}\left(\varepsilon_{1}, \varepsilon_{2}\right) g_{22}\left(\varepsilon_{1}, \varepsilon_{2}\right)\right) \quad(\in \mathbb{R}), \\
\varrho_{2}\left(\varepsilon_{1}, \varepsilon_{2}\right):=\varrho_{1}\left(\varepsilon_{1}, \varepsilon_{2}\right) \ln (24 \pi)+(-1)^{\varepsilon_{2}} \operatorname{Re}(a) \ln (2+\sqrt{3})+\frac{\pi}{2}-\frac{1}{2} \arg \left(\frac{g_{11}\left(\varepsilon_{1}, \varepsilon_{2}\right) g_{12}\left(\varepsilon_{1}, \varepsilon_{2}\right)}{g_{21}\left(\varepsilon_{1}, \varepsilon_{2}\right) g_{22}\left(\varepsilon_{1}, \varepsilon_{2}\right)}\right) \\
-\arg \left(\Gamma\left(\frac{1}{2}+\mathrm{i} \varrho_{1}\left(\varepsilon_{1}, \varepsilon_{2}\right)\right)\right)+\mathrm{i}\left((-1)^{\varepsilon_{2}} \operatorname{Im}(a) \ln (2+\sqrt{3})+\frac{1}{2} \ln \left(\left|\frac{g_{11}\left(\varepsilon_{1}, \varepsilon_{2}\right) g_{12}\left(\varepsilon_{1}, \varepsilon_{2}\right)}{g_{21}\left(\varepsilon_{1}, \varepsilon_{2}\right) g_{22}\left(\varepsilon_{1}, \varepsilon_{2}\right)}\right|\right)\right),
\end{gathered}
$$

and $\vartheta_{0}$ is given in Equation (2.7).

Proof. Follows from a successive approximations argument applied to Equations (4.29).

Proposition 4.5. Let the conditions on the monodromy data be the same as those in Proposition 4.4 . Suppose that $0<13 \delta<\delta_{1}<1 / 3$. Then

$$
0<\delta<\delta_{G}<\frac{\delta_{1}-8 \delta}{5}
$$

Proof. Consider the definition of $\delta_{G}$ given in Equation (3.144), where $\delta^{0}$ is defined in Theorem 3.4.1 and Lemma 3.4.2, $\delta_{12}^{\infty}$ and $\delta_{21}^{\infty}$ are defined in Lemma 3.4.1, and $\delta_{12}^{G}=\delta_{21}^{G}=\delta^{0}$ (cf. Equations (3.143) and (4.19) ). Under the assumptions stated in the Proposition, one can choose $\varepsilon$ which appears in these equations to satisfy the inequalities

$$
\frac{3 \delta}{2}<\varepsilon<\frac{\delta_{1}+2 \delta}{10}
$$

then $\delta_{G}=2(\varepsilon-\delta)$.

This completes the proof of Theorem 2.3 modulo the distribution of the cheese-holes in $\widetilde{\mathcal{D}}_{u}$.

${ }^{15} \tau_{a s, m}^{\infty}, \tau_{a s, m}^{+}$, and $\tau_{a s, m}^{-}$are the poles and zeroes of the leading term of asymptotics of $u(\tau)$ (cf. Equation (2.4)). 
Theorem 4.1. For any given solution $u(\tau)$ with the monodromy data satisfying the conditions (2.11), consider the cheese-like domain $\mathcal{D}_{u}$ (cf. Remark 2.4). There exists $T>0$ such that, $\forall \tau_{a s, m}^{ \pm}, \tau_{a s, m}^{\infty}$ with $\left|\tau_{a s, m}^{ \pm}\right|>T$ and $\left|\tau_{a s, m}^{\infty}\right|>T$ in each cheese-hole centered at $\tau_{a s, m}^{ \pm}\left(\right.$or $\left.\tau_{a s, m}^{\infty}\right)$, there exists one, and only one, zero $\tau_{m}^{ \pm}$(or pole $\tau_{m}^{\infty}$ ) of $u(\tau)$ located in this cheese-hole:

$$
\left|\tau_{a s, m}^{ \pm}-\tau_{m}^{ \pm}\right|<C\left|\tau_{a s, m}^{ \pm}\right|^{\frac{1}{3}-\delta}, \quad\left|\tau_{a s, m}^{\infty}-\tau_{m}^{\infty}\right|<C\left|\tau_{a s, m}^{\infty}\right|^{\frac{1}{3}-\delta}
$$

where $C>0$.

Proof. As in the main body of the paper, the case $\varepsilon_{1}=\varepsilon_{2}=0$ is considered in detail; in particular, $\vartheta=\vartheta(0,0, \tau)=\widetilde{\vartheta}(\tau)+\vartheta_{0}-\pi$ : the remaining cases are proved analogously upon applying the transformation $\mathcal{F}_{\varepsilon_{1}, \varepsilon_{2}}$ introduced at the beginning of Section 2 . The strategy here is to integrate both sides of Equations (4.27) and (2.18), and the equation resulting upon multiplying Equation (2.19) by the factor $2 \tau^{-1}$, around the boundary of a cheese-hole centered at $\tau_{*}=\tau_{a s, m}^{ \pm}\left(\right.$or $\left.\tau_{a s, m}^{\infty}\right)$. Hereafter, the symbol $\oint$ means that the above-mentioned integration is taken in the counter-clockwise sense.

Denote by $n_{+}, n_{-}$, and $n_{p}$, respectively, the numbers of zeroes of types $\tau_{+}, \tau_{-}$, and poles $\tau_{p}$ of a solution $u(\tau)$ inside the cheese-hole around whose boundary the integration is performed. The integrals on the left-hand sides of Equations (4.27) and (2.18), and the equation resulting upon multiplying Equation (2.19) by the factor $2 \tau^{-1}$, can be calculated via the Cauchy Residue Theorem; the results, respectively, read:

$$
2 \pi \mathrm{i}\left(n_{+}+n_{-}-2 n_{p}\right), \quad 2 \pi \mathrm{i}\left(n_{-}+n_{p}\right), \quad 2 \pi \mathrm{i}\left(n_{-}-n_{p}\right) .
$$

To integrate the right-hand sides, note that the error estimates in the above-mentioned equations are all of the form $\mathcal{O}\left(\tau^{-\frac{1}{3}-v}\right)$, for some $v>0$; therefore,

$$
\oint \mathcal{O}\left(\frac{1}{\tau^{\frac{1}{3}+v}}\right) \mathrm{d} \tau \underset{\tau \rightarrow \infty}{\sim} \mathcal{O}\left(\frac{C}{\tau_{*}^{v+\delta}}\right) \underset{\tau_{*} \rightarrow \infty}{\rightarrow} 0 .
$$

Thus, for some $T>0$, the integral is less than $2 \pi / 3$. The leading terms of the right-hand sides of Equations (4.27) and (2.18), and the equation resulting upon multiplying Equation (2.19) by the factor $2 \tau^{-1}$, are meromorphic functions in the strip $\mathcal{D}$, where $\mathcal{D}$ is defined by Equation (2.10); therefore, one can also calculate $\oint$ by using the Cauchy Residue Theorem 16. To do so, notice that the non-trivial part of the leading terms (non-vanishing under integration) has the form $\tau^{-1 / 3} F(\vartheta)$, where the function $F$ is expressed in terms of trigonometric functions:

$$
\begin{aligned}
\oint 2 \sqrt{3}(\epsilon b)^{1 / 3} \tau^{-1 / 3} F(\vartheta) \mathrm{d} \tau & =\oint \hat{\vartheta}^{\prime} F(\vartheta) \mathrm{d} \tau+\mathcal{O}\left(\oint \frac{F(\vartheta)}{\tau} \mathrm{d} \tau\right) \\
& =\oint \hat{\vartheta}^{\prime} F(\hat{\vartheta}) \mathrm{d} \tau+\mathcal{O}\left(\oint \hat{\vartheta}^{\prime} \max \left(\left|F^{\prime}(\hat{\vartheta})\right|\right) \tau^{-\delta_{G}} \ln \tau \mathrm{d} \tau\right)+\mathcal{O}\left(\oint \frac{F(\vartheta)}{\tau} \mathrm{d} \tau\right) \\
& =\oint F(\hat{\vartheta}) \mathrm{d} \hat{\vartheta}+\mathcal{O}\left(\tau_{*}^{\delta-\delta_{G}} \ln \tau_{*}\right)+\mathcal{O}\left(\tau_{*}^{-2 / 3}\right)
\end{aligned}
$$

where use has been made of the fact that $\delta<\delta_{G}, \max \left(\left|F^{\prime}(\hat{\vartheta})\right|\right)=\mathcal{O}\left(\tau_{*}^{2 \delta}\right)$, and $F(\vartheta)=\mathcal{O}\left(\tau_{*}^{\delta}\right)$ in the annular region of width $\mathcal{O}\left(\tau^{-\delta_{G}} \ln \tau\right)$ around the circle of integration. Again, for $\tau_{*}>T$, each estimate in Equation (4.35) is less than $2 \pi / 3$; thus, for large enough $\tau_{*}$, the modulus of the contribution of the error estimates to $\oint$ is less than $2 \pi$. (Their contribution is actually equal to zero, since the results of the integration of the right-hand sides should coincide with the results of the integration of the corresponding left-hand sides, and, according to Equations (4.34), should be equal be $2 \pi \mathrm{i}$ multipled by an integer.) Now, calculating the integrals $\oint F(\hat{\vartheta}) \mathrm{d} \hat{\vartheta}$ in Equations (4.27) and (2.18), and the equation resulting upon multiplying Equation (2.19) by the factor $2 \tau^{-1}$, one arrives at the following systems: (i) for $\tau_{*}=\tau_{a s, m}^{\infty}$,

$$
2 \pi \mathrm{i}\left(n_{+}+n_{-}-2 n_{p}\right)=-4 \pi \mathrm{i}, \quad 2 \pi \mathrm{i}\left(n_{-}+n_{p}\right)=2 \pi \mathrm{i}, \quad 2 \pi \mathrm{i}\left(n_{-}-n_{p}\right)=-2 \pi \mathrm{i} ;
$$

(ii) for $\tau_{*}=\tau_{a s, m}^{+}$,

$$
2 \pi \mathrm{i}\left(n_{+}+n_{-}-2 n_{p}\right)=2 \pi \mathrm{i}, \quad 2 \pi \mathrm{i}\left(n_{-}+n_{p}\right)=0, \quad 2 \pi \mathrm{i}\left(n_{-}-n_{p}\right)=0 ;
$$

\footnotetext{
${ }^{16}$ It follows from the derivation in Section 3 and this section that the error estimate $\mathcal{O}\left(\tau^{-\delta_{G}} \ln \tau\right)$ in the "phase", $\vartheta$, is a meromorphic function in $\mathcal{D}$; this fact, however, is not used in the proof.
} 
and (iii) for $\tau_{*}=\tau_{a s, m}^{-}$,

$$
2 \pi \mathrm{i}\left(n_{+}+n_{-}-2 n_{p}\right)=2 \pi \mathrm{i}, \quad 2 \pi \mathrm{i}\left(n_{-}+n_{p}\right)=2 \pi \mathrm{i}, \quad 2 \pi \mathrm{i}\left(n_{-}-n_{p}\right)=2 \pi \mathrm{i} .
$$

One completes the proof upon solving these systems.

Corollary 4.1. Define $\hat{\tau}_{a s, m}^{\infty}$ and $\hat{\tau}_{a s, m}^{ \pm}$, respectively, as the leading terms of asymptotics of $\tau_{a s, m}^{\infty}$ and $\tau_{a s, m}^{ \pm}$(cf. Proposition 4.4), that is,

$$
\tau_{a s, m}^{\infty}=: \hat{\tau}_{a s, m}^{\infty}+\mathcal{O}\left(\frac{\ln ^{2} m}{\sqrt{m}}\right), \quad \tau_{a s, m}^{ \pm}=: \hat{\tau}_{a s, m}^{ \pm}+\mathcal{O}\left(\frac{\ln ^{2} m}{\sqrt{m}}\right)
$$

Under the conditions of Theorem 4.1,

$$
\tau_{m}^{\infty} \underset{m \rightarrow \infty}{=} \hat{\tau}_{a s, m}^{\infty}+\mathcal{O}\left(m^{\frac{1}{2}-\frac{3 \delta}{2}}\right), \quad \tau_{m}^{ \pm} \underset{m \rightarrow \infty}{=} \hat{\tau}_{a s, m}^{ \pm}+\mathcal{O}\left(m^{\frac{1}{2}-\frac{3 \delta}{2}}\right),
$$

where $0<\delta<1 / 39$.

Proof. As follows from Proposition 4.5, $0<\delta<1 / 39$; thus, $\ln ^{2} m / \sqrt{m} \ll m^{\frac{1}{2}-\frac{3 \delta}{2}}$ as $m \rightarrow \infty$. in $\widetilde{\mathcal{D}}_{u}$.

This completes the proof of Theorem 2.2, which is equivalent to the specification of the cheese-holes

\section{Acknowledgments}

The authors were supported, in part, by a College of Charleston (CofC) Mathematics Department Summer Research Award. 


\section{Appendix A: Poles and Zeroes}

In this Appendix some basic information concerning the poles and zeroes of the solution $u(\tau)$ of Equation (1.1) and the associated functions $\mathcal{H}(\tau)$ and $f(\tau)$ (cf. Equations (1.2) and (1.3), respectively) are presented.

Let $\tau_{\infty}$ be a pole of $u(\tau)$; then, its Laurent expansion reads

$$
u(\tau)=-\frac{\tau_{\infty}}{4 \epsilon\left(\tau-\tau_{\infty}\right)^{2}}+a_{0}+\sum_{k=1}^{\infty} a_{k}\left(\tau-\tau_{\infty}\right)^{k}, \quad \epsilon= \pm 1
$$

where $a_{0}$ is a parameter, and the remaining coefficients, $a_{k}$, are recursively and uniquely defined; for example,

$$
\begin{gathered}
a_{1}=-\frac{a_{0}}{\tau_{\infty}}, \quad a_{2}=\frac{a b}{5 \tau_{\infty}}-\frac{12 \epsilon a_{0}^{2}}{5 \tau_{\infty}}+\frac{9 a_{0}}{10 \tau_{\infty}^{2}}, \quad a_{3}=-\frac{8 a b}{45 \tau_{\infty}^{2}}+\frac{24 \epsilon a_{0}^{2}}{5 \tau_{\infty}^{2}}-\frac{4 a_{0}}{5 \tau_{\infty}^{3}}, \\
a_{4}=-\frac{\epsilon b^{2}}{7 \tau_{\infty}}+\frac{32 a_{0}^{3}}{7 \tau_{\infty}^{2}}-\frac{4 \epsilon a_{0} a b}{7 \tau_{\infty}^{2}}+\frac{10 a b}{63 \tau_{\infty}^{3}}-\frac{47 \epsilon a_{0}^{2}}{7 \tau_{\infty}^{3}}+\frac{5 a_{0}}{7 \tau_{\infty}^{4}} \\
a_{5}=\frac{\epsilon b^{2}}{35 \tau_{\infty}^{2}}-\frac{96 a_{0}^{3}}{7 \tau_{\infty}^{3}}+\frac{124 \epsilon a_{0} a b}{105 \tau_{\infty}^{3}}-\frac{a b}{7 \tau_{\infty}^{4}}+\frac{57 \epsilon a_{0}^{2}}{7 \tau_{\infty}^{4}}-\frac{9 a_{0}}{14 \tau_{\infty}^{5}}
\end{gathered}
$$

The Laurent expansion of the associated Hamiltonian, $\mathcal{H}(\tau)$, reads

$$
\mathcal{H}(\tau)-\frac{1}{2 \tau}\left(a-\frac{\mathrm{i}}{2}\right)^{2}=\frac{1}{\tau-\tau_{\infty}}+H_{0}+\sum_{k=1}^{\infty} H_{k}\left(\tau-\tau_{\infty}\right)^{k}
$$

where the coefficients, $H_{k}$, can be uniquely determined via Equation (37) of [1]; for example,

$$
\begin{gathered}
H_{0}=12 \epsilon a_{0}, \quad H_{1}=-\frac{8 \epsilon a_{0}}{\tau_{\infty}}, \quad H_{2}=\frac{2 \mathrm{i} \epsilon b}{\tau_{\infty}}+\frac{6 \epsilon a_{0}}{\tau_{\infty}^{2}} \\
H_{3}=-\frac{16 a \epsilon b}{15 \tau_{\infty}^{2}}-\frac{16 a_{0}^{2}}{5 \tau_{\infty}^{2}}-\frac{24 \epsilon a_{0}}{5 \tau_{\infty}^{3}}, \quad H_{4}=\frac{8 \mathrm{i} b a_{0}}{\tau_{\infty}^{2}}+\frac{8 a \epsilon b}{9 \tau_{\infty}^{3}}+\frac{8 a_{0}^{2}}{\tau_{\infty}^{3}}+\frac{4 \epsilon a_{0}}{\tau_{\infty}^{4}} \\
H_{5}=\frac{52 b^{2}}{35 \tau_{\infty}^{2}}-\frac{128 a b a_{0}}{35 \tau_{\infty}^{3}}+\frac{128 \epsilon a_{0}^{3}}{35 \tau_{\infty}^{3}}-\frac{8 \mathrm{i} b a_{0}}{\tau_{\infty}^{3}}-\frac{16 a \epsilon b}{21 \tau_{\infty}^{4}}-\frac{468 a_{0}^{2}}{35 \tau_{\infty}^{4}}-\frac{24 \epsilon a_{0}}{7 \tau_{\infty}^{5}}
\end{gathered}
$$

The function $f(\tau)$ also has a first-order pole at $\tau=\tau_{\infty}$ :

$$
f(\tau)=-\frac{\tau_{\infty}}{2\left(\tau-\tau_{\infty}\right)}+f_{0}+\sum_{k=1}^{\infty} f_{k}\left(\tau-\tau_{\infty}\right)^{k}
$$

where the coefficients, $f_{k}$, can be uniquely determined via Equation (1.3); for example,

$$
\begin{gathered}
f_{0}=-\frac{\mathrm{i} a}{2}-\frac{3}{4}, \quad f_{1}=-2 a_{0} \epsilon, \quad f_{2}=\mathrm{i} \epsilon b+\frac{a_{0} \epsilon}{\tau_{\infty}} \\
f_{3}=\frac{8 a_{0}^{2}}{5 \tau_{\infty}}-\frac{4 a \epsilon b}{5 \tau_{\infty}}+\frac{\mathrm{i} \epsilon b}{\tau_{\infty}}-\frac{3 a_{0} \epsilon}{5 \tau_{\infty}^{2}}, \quad f_{4}=\frac{4 \mathrm{i} a_{0} b}{\tau_{\infty}}-\frac{12 a_{0}^{2}}{5 \tau_{\infty}^{2}}+\frac{4 a \epsilon b}{45 \tau_{\infty}^{2}}+\frac{2 a_{0} \epsilon}{5 \tau_{\infty}^{3}} \\
f_{5}=\frac{6 b^{2}}{7 \tau_{\infty}}-\frac{64 \epsilon a_{0}^{3}}{35 \tau_{\infty}^{2}}-\frac{48 a b a_{0}}{35 \tau_{\infty}^{2}}-\frac{4 a \epsilon b}{63 \tau_{\infty}^{3}}+\frac{94 a_{0}^{2}}{35 \tau_{\infty}^{3}}-\frac{2 \epsilon a_{0}}{7 \tau_{\infty}^{4}}
\end{gathered}
$$

There are two types of first-order zeroes of $u(\tau)$, denoted $\tau_{s}, s= \pm 1$, which differ by their Taylor expansions:

$$
u(\tau)=\sum_{k=1}^{\infty} b_{k}^{s}\left(\tau-\tau_{s}\right)^{k}
$$

where, for example,

$$
b_{1}^{s}=\mathrm{i} s b, \quad b_{2}^{s}=-\frac{b}{\tau_{s}}\left(a-\frac{\mathrm{i} s}{2}\right), \quad b_{3}^{s} \in \mathbb{C}, \quad b_{4}^{s}=\frac{1}{2 \tau_{s}}\left(4 \epsilon b^{2}+\mathrm{i} s a b_{3}^{s}-b_{3}^{s}\right),
$$




$$
b_{5}^{s}=\frac{1}{20 b \tau_{s}^{2}}\left(7 b b_{3}^{s}-8 b^{3} \epsilon-6 \mathrm{i} s\left(b_{3}^{s}\right)^{2} \tau_{s}^{2}+48 \mathrm{i} s a b^{3} \epsilon-10 \mathrm{i} s a b b_{3}^{s}\right) .
$$

These two types of first-order zeroes are "analytically" distinguished by the Hamiltonian function; indeed, at $\tau=\tau_{+}$, the function $\mathcal{H}(\tau)$ is holomorphic,

$$
\mathcal{H}(\tau)=H_{0}^{+}+\sum_{k=1}^{\infty} H_{k}^{+}\left(\tau-\tau_{+}\right)^{k},
$$

where the first few coefficients are

$$
\begin{gathered}
H_{0}^{+}=-\frac{3 \mathrm{i} b_{3}^{+} \tau_{+}}{2 b}+\frac{1}{2 \tau_{+}}\left(a-\frac{\mathrm{i}}{2}\right)^{2}+\frac{\mathrm{i}}{\tau_{+}}\left(a-\frac{\mathrm{i}}{2}\right), \quad H_{1}^{+}=\frac{1}{2 \tau_{+}^{2}}\left(a-\frac{\mathrm{i}}{2}\right)^{2}, \\
H_{2}^{+}=-\frac{3 b_{3}^{+}}{2 b \tau_{+}}\left(a-\frac{\mathrm{i}}{2}\right)+\frac{1}{2 \tau_{+}^{3}}\left(a-\frac{\mathrm{i}}{2}\right)^{2}-\frac{\mathrm{i}}{\tau_{+}^{3}}\left(a-\frac{\mathrm{i}}{2}\right)^{3}
\end{gathered}
$$

while, at $\tau=\tau_{-}$, the function $\mathcal{H}(\tau)$ has a first-order pole,

$$
\mathcal{H}(\tau)-\frac{1}{2 \tau}\left(a-\frac{\mathrm{i}}{2}\right)^{2}=\frac{1}{\tau-\tau_{-}}+H_{0}^{-}+\sum_{k=1}^{\infty} H_{k}^{-}\left(\tau-\tau_{-}\right)^{k},
$$

where, for example,

$$
H_{0}^{-}=\frac{3 \mathrm{i} b_{3}^{-} \tau_{-}}{2 b}, \quad H_{1}^{-}=-\frac{\mathrm{i} b_{3}^{-}}{b}, \quad H_{2}^{-}=-\frac{2 \mathrm{i} \epsilon b}{\tau_{-}}+\frac{3 \mathrm{i} b_{3}^{-}}{4 b \tau_{-}} .
$$

The zeroes of $u(\tau)$ are also distinguished by the function $f(\tau)$ :

$$
f(\tau)=\sum_{k=1}^{\infty} f_{k}^{+}\left(\tau-\tau_{+}\right)^{k}
$$

where the first two coefficients are

$$
f_{1}^{+}=\frac{4 a^{2}+1}{8 \tau_{+}}-\frac{3 \mathrm{i} b_{3}^{+} \tau_{+}}{4 b}, \quad f_{2}^{+}=-2 \mathrm{i} \epsilon b-\frac{3 b_{3}^{+}}{4 b}\left(a-\frac{\mathrm{i}}{2}\right)-\frac{\mathrm{i}\left(4 a^{2}+1\right)}{8 \tau_{+}^{2}}\left(a-\frac{\mathrm{i}}{2}\right)
$$

and

$$
f(\tau)=\frac{\tau_{-}}{2\left(\tau-\tau_{-}\right)}+f_{0}^{-}+\sum_{k=1}^{\infty} f_{k}^{-}\left(\tau-\tau_{-}\right)^{k}
$$

where, for example,

$$
f_{0}^{-}=-\frac{\mathrm{i}}{2}\left(a+\frac{\mathrm{i}}{2}\right), \quad f_{1}^{-}=\frac{\mathrm{i} b_{3}^{-} \tau_{-}}{4 b}, \quad f_{2}^{-}=\mathrm{i} \epsilon b-\frac{\mathrm{i} b_{3}^{-}}{8 b} .
$$

As follows from the definitions of the associated functions $\mathcal{H}(\tau)$ and $f(\tau)$, they have poles only at the poles and $\tau_{-}$-zeroes of the function $u(\tau)$.

\section{Appendix B: Comparison of Asymptotic Results}

The ranges of the validity for asymptotics of $u(\tau)$ obtained in Part I [1] of our studies and in this paper overlap: this enables us to resolve the ambiguity in the choice of sign for the function $\hat{r}_{0}(\tau)$ discussed in the proof of Proposition 4.1.

The asymptotics of $u(\tau)$ for $\left|\operatorname{Re}\left(\widetilde{\nu}\left(\varepsilon_{1}, \varepsilon_{2}\right)+1\right)\right|<1 / 6, \varepsilon_{1}, \varepsilon_{2}=0, \pm 1$, are obtained in Theorem 3.1 of [1, whilst the asymptotics of $u(\tau)$ stated in Theorem 2.1 of this paper are applicable for $\operatorname{Re}\left(\widetilde{\nu}\left(\varepsilon_{1}, \varepsilon_{2}\right)+\right.$ $1) \in(0,1) \backslash\{1 / 2\}$. In fact, if one is concerned only with the leading exponent of $\cosh (\cdot)$ in the asymptotics of $u(\tau)$ presented in Theorem 3.1 of [1], then the range of its validity can be extended to $\left|\operatorname{Re}\left(\widetilde{\nu}\left(\varepsilon_{1}, \varepsilon_{2}\right)+1\right)\right|<1 / 2$. Analogously, only the leading exponent in the expansion of $\sin ^{-2}(\cdot)$ in the asymptotics of $u(\tau)$ obtained in Theorem 2.1 of this paper is "larger" than the error correction term 
in case $\operatorname{Re}\left(\widetilde{\nu}\left(\varepsilon_{1}, \varepsilon_{2}\right)+1\right)>0$. Therefore, the corresponding leading exponents of the asymptotics of $u(\tau)$ in Theorem 3.1 of [1] and Theorem 2.1 of this paper should coincide, provided

$$
0<\operatorname{Re}\left(\widetilde{\nu}\left(\varepsilon_{1}, \varepsilon_{2}\right)+1\right)<\frac{1}{2} .
$$

Recall the main asymptotic (as $\tau \rightarrow \infty$ ) result for $u(\tau)$ obtained in Part I [1] 17 :

Theorem B.1 (Theorem $3.1[1]$ ). Let $\varepsilon_{1}, \varepsilon_{2}=0, \pm 1, \epsilon b=|\epsilon b| \mathrm{e}^{\mathrm{i} \pi \varepsilon_{2}}$, and $u(\tau)$ be a solution of the degenerate third Painlevé equation (1.1) corresponding to the monodromy data $\left(a, s_{0}^{0}, s_{0}^{\infty}, s_{1}^{\infty}, g_{11}, g_{12}, g_{21}\right.$, $\left.g_{22}\right)$. Suppose that

$$
g_{11}\left(\varepsilon_{1}, \varepsilon_{2}\right) g_{12}\left(\varepsilon_{1}, \varepsilon_{2}\right) g_{21}\left(\varepsilon_{1}, \varepsilon_{2}\right) g_{22}\left(\varepsilon_{1}, \varepsilon_{2}\right) \neq 0, \quad\left|\operatorname{Re}\left(\frac{\mathrm{i}}{2 \pi} \ln \left(g_{11}\left(\varepsilon_{1}, \varepsilon_{2}\right) g_{22}\left(\varepsilon_{1}, \varepsilon_{2}\right)\right)\right)\right|<\frac{1}{6}
$$

Then, $\exists \delta>0$ such that $u(\tau)$ has the asymptotic expansion

$$
\begin{aligned}
& u(\tau) \underset{\tau \rightarrow \infty \mathrm{e}^{\mathrm{i} \pi \varepsilon_{1}}}{=} \frac{(-1)^{\varepsilon_{1}} \epsilon \sqrt{|\epsilon b|}}{3^{1 / 4}}\left(\sqrt{\frac{\vartheta(\tau)}{12}}+\sqrt{\widetilde{\nu}\left(\varepsilon_{1}, \varepsilon_{2}\right)+1} \mathrm{e}^{\frac{3 \pi \mathrm{i}}{4}} \cosh \left(\mathrm{i} \vartheta(\tau)+\left(\widetilde{\nu}\left(\varepsilon_{1}, \varepsilon_{2}\right)+1\right) \ln \vartheta(\tau)\right.\right. \\
& \left.\left.\quad+z\left(\varepsilon_{1}, \varepsilon_{2}\right)+o\left(\tau^{-\delta}\right)\right)\right),
\end{aligned}
$$

where

$$
\begin{aligned}
\vartheta(\tau) & :=3 \sqrt{3}|\epsilon b|^{1 / 3}|\tau|^{2 / 3}, \quad \widetilde{\nu}\left(\varepsilon_{1}, \varepsilon_{2}\right)+1:=\frac{\mathrm{i}}{2 \pi} \ln \left(g_{11}\left(\varepsilon_{1}, \varepsilon_{2}\right) g_{22}\left(\varepsilon_{1}, \varepsilon_{2}\right)\right), \\
z\left(\varepsilon_{1}, \varepsilon_{2}\right) & :=\frac{1}{2 \pi} \ln 2 \pi-\frac{\mathrm{i} \pi}{2}-\frac{3 \pi \mathrm{i}}{2}\left(\widetilde{\nu}\left(\varepsilon_{1}, \varepsilon_{2}\right)+1\right)+(-1)^{\varepsilon_{2}} \mathrm{i} a \ln (2+\sqrt{3})+\left(\widetilde{\nu}\left(\varepsilon_{1}, \varepsilon_{2}\right)+1\right) \ln 12 \\
& -\ln \left(\omega\left(\varepsilon_{1}, \varepsilon_{2}\right) \sqrt{\widetilde{\nu}\left(\varepsilon_{1}, \varepsilon_{2}\right)+1} \Gamma\left(\widetilde{\nu}\left(\varepsilon_{1}, \varepsilon_{2}\right)+1\right)\right),
\end{aligned}
$$

with

$$
\omega\left(\varepsilon_{1}, \varepsilon_{2}\right):=\frac{g_{12}\left(\varepsilon_{1}, \varepsilon_{2}\right)}{g_{22}\left(\varepsilon_{1}, \varepsilon_{2}\right)}
$$

and $\Gamma(\cdot)$ is the gamma function.

Let $\mathcal{H}(\tau)$ be the Hamiltonian function defined in Equation (1.2) corresponding to the function $u(\tau)$ given above. Then,

$$
\begin{gathered}
\mathcal{H}(\tau)-\frac{1}{2 \tau}\left(a-\frac{(-1)^{\varepsilon_{2}} \mathrm{i}}{2}\right)^{2} \underset{\tau \rightarrow \infty \mathrm{e}^{\mathrm{i} \pi \varepsilon_{1}}}{ } 3(\epsilon b)^{2 / 3} \tau^{1 / 3}+2|\epsilon b|^{1 / 3} \tau^{-1 / 3}\left(\left(a-\frac{(-1)^{\varepsilon_{2}} \mathrm{i}}{2}\right)\right. \\
\left.-\mathrm{i} 2 \sqrt{3}\left(\widetilde{\nu}\left(\varepsilon_{1}, \varepsilon_{2}\right)+1\right)+o\left(\tau^{-\delta}\right)\right) .
\end{gathered}
$$

Now, taking into account condition (B.1), one shows that asymptotics (B.2) implies

$$
\begin{aligned}
u(\tau) \underset{\tau \rightarrow \infty \mathrm{e}^{\mathrm{i} \pi \varepsilon_{1}}}{ } \frac{(-1)^{\varepsilon_{2}} \epsilon|\epsilon b|^{2 / 3}}{2}|\tau|^{1 / 3}+\frac{(-1)^{\varepsilon_{1}} \epsilon \sqrt{|\epsilon b|}}{2 \cdot 3^{1 / 4}}|\tau|^{\frac{2}{3} \operatorname{Re}\left(\widetilde{\nu}\left(\varepsilon_{1}, \varepsilon_{2}\right)+1\right)} \mathrm{e}^{\Theta(\tau)}+\mathcal{O}\left(\tau^{-\frac{2}{3} \operatorname{Re}\left(\widetilde{\nu}\left(\varepsilon_{1}, \varepsilon_{2}\right)+1\right)}\right) \\
\quad+o\left(\tau^{\frac{2}{3} \operatorname{Re}\left(\widetilde{\nu}\left(\varepsilon_{1}, \varepsilon_{2}\right)+1\right)-\delta}\right)
\end{aligned}
$$

where

$$
\begin{aligned}
\Theta(\tau) & :=\mathrm{i} 3 \sqrt{3}|\epsilon b|^{1 / 3}|\tau|^{2 / 3}+\mathrm{i} \operatorname{Im}\left(\widetilde{\nu}\left(\varepsilon_{1}, \varepsilon_{2}\right)+1\right) \ln |\tau|^{2 / 3}+\left(\widetilde{\nu}\left(\varepsilon_{1}, \varepsilon_{2}\right)+1\right) \ln \left(36 \sqrt{3}|\epsilon b|^{1 / 3}\right) \\
& +(-1)^{\varepsilon_{2}} \mathrm{i} a \ln (2+\sqrt{3})+\frac{\mathrm{i} \pi}{4}-\frac{3 \pi \mathrm{i}}{2}\left(\widetilde{\nu}\left(\varepsilon_{1}, \varepsilon_{2}\right)+1\right)-\ln \left(\frac{\omega\left(\varepsilon_{1}, \varepsilon_{2}\right) \Gamma\left(\widetilde{\nu}\left(\varepsilon_{1}, \varepsilon_{2}\right)+1\right)}{\sqrt{2 \pi}}\right) .
\end{aligned}
$$

Using the Euler formula for $\sin (\cdot)$ to expand asymptotics (2.3) (assuming condition (B.1) in Theorem 2.1 of this work, one arrives, again, at asymptotics (B.4), but with the error correction

$$
\mathcal{O}\left(\tau^{\frac{2}{3} \operatorname{Re}\left(\widetilde{\nu}\left(\varepsilon_{1}, \varepsilon_{2}\right)+1\right)-\delta_{G}} \ln \tau\right)+\mathcal{O}\left(\tau^{\frac{4}{3} \operatorname{Re}\left(\widetilde{\nu}\left(\varepsilon_{1}, \varepsilon_{2}\right)+1\right)-\frac{1}{3}}\right)
$$

\footnotetext{
${ }^{17}$ In order to facilitate the comparison, the parameter $\varepsilon$ which appears in Theorem 3.1 of [1] has been changed to $\epsilon$.
} 
furthermore, there is a discrepancy in the definition of the parameter $\omega\left(\varepsilon_{1}, \varepsilon_{2}\right)$ (cf. Equation (B.5)): instead of (B.3), one finds that

$$
\omega\left(\varepsilon_{1}, \varepsilon_{2}\right):=g_{11}\left(\varepsilon_{1}, \varepsilon_{2}\right) g_{12}\left(\varepsilon_{1}, \varepsilon_{2}\right) .
$$

In fact, (B.6) is the correct definition of $\omega\left(\varepsilon_{1}, \varepsilon_{2}\right)$ (see the discussion below); therefore, (B.6) should be used in lieu of the definition of $\omega\left(\varepsilon_{1}, \varepsilon_{2}\right)$ in Theorem B.1 and in Theorem 3.1 of [1].

The root of this discrepancy is related with the fact that the right factor of the second term in Equation (79) of [1] should be changed, namely,

$$
\ln \left(\frac{3(\varepsilon b)^{1 / 6}}{\sqrt{2}}\right) \rightarrow \ln \left(\frac{3(\varepsilon b)^{1 / 6} \mathrm{e}^{-\mathrm{i} \pi}}{\sqrt{2}}\right) .
$$

As a consequence of (B.7), corresponding changes should be made to the following formulae in 11: (i) to the right-hand sides of Equations (96), (98), and (99) one must add the term $\mathrm{i} \pi(\nu+1)$; (ii) to the argument of the exponential function on the right-hand side of Equation (101) one must add the term $-2 \pi \mathrm{i}(\nu+1)$; (iii) to the right-hand side of the formula for $z_{n}$ given in Corollary 4.3 .1 one must add the term $2 \pi \mathrm{i}(\nu+1)$; and (iv) the definition of the parameter $\widehat{\omega}\left(\varepsilon_{1}, \varepsilon_{2}\right)$ appearing in Theorem A.1 should be changed 18 to $\widehat{\omega}\left(\varepsilon_{1}, \varepsilon_{2}\right):=\widehat{g}_{11}\left(\varepsilon_{1}, \varepsilon_{2}\right) \widehat{g}_{12}\left(\varepsilon_{1}, \varepsilon_{2}\right)$.

Making analogous expansions for the $\cot (\cdot)$ functions in Equation (2.8) of Theorem 2.1, one obtains an asymptotic formula for $\mathcal{H}(\tau)$ that coincides with the one presented in Theorem B.1

For the other choice of $\hat{r}_{0}(\tau)$ (cf. the proof of Proposition 4.1) one would obtain the same asymptotics (B.4) - B.5) but with the change $\Theta(\tau) \rightarrow \Theta(\tau)+\mathrm{i} \pi$.

\section{Appendix C: Asymptotics for Imaginary $\tau$}

Here, asymptotics as $\tau \rightarrow \pm \mathrm{i} \infty$ of the functions $u(\tau), \mathcal{H}(\tau)$, and $f(\tau)$ are presented. These results are obtained by applying transformations 6.2.2 (changing $19 a \rightarrow-a$ ) and 6.2.3 (changing 20 $\tau \rightarrow \mathrm{i} \tau$ ) given in Section 6 of [1] to the asymptotic results (for $\varepsilon_{1}=\varepsilon_{2}=0$ ) stated in Theorems 2.1 2.3 of this paper. For this purpose, it is convenient to introduce the auxiliary mapping 21

$$
\begin{gathered}
\widehat{\mathcal{F}}_{\varepsilon_{1}, \varepsilon_{2}}: \mathcal{M} \rightarrow \mathcal{M}, \quad\left(a, s_{0}^{0}, s_{0}^{\infty}, s_{1}^{\infty}, g_{11}, g_{12}, g_{21}, g_{22}\right) \mapsto \\
\left((-1)^{1+\varepsilon_{2}} a, s_{0}^{0}, \widehat{s}_{0}^{\infty}\left(\varepsilon_{1}, \varepsilon_{2}\right), \widehat{s}_{1}^{\infty}\left(\varepsilon_{1}, \varepsilon_{2}\right), \widehat{g}_{11}\left(\varepsilon_{1}, \varepsilon_{2}\right), \widehat{g}_{12}\left(\varepsilon_{1}, \varepsilon_{2}\right), \widehat{g}_{21}\left(\varepsilon_{1}, \varepsilon_{2}\right), \widehat{g}_{22}\left(\varepsilon_{1}, \varepsilon_{2}\right)\right), \varepsilon_{1}= \pm 1, \varepsilon_{2}=0, \pm 1,
\end{gathered}
$$

which is equivalent to the formulae for the monodromy data in transformations 6.2.2 and 6.2.3 in Section 6 of [1] (with $l=\varepsilon_{1}$ and $p=\varepsilon_{2}$ ). Define22:

(1) $\widehat{\mathcal{F}}_{-1,0}: \widehat{s}_{0}^{\infty}(-1,0)=s_{1}^{\infty} \mathrm{e}^{\frac{3 \pi a}{2}}, \widehat{s}_{1}^{\infty}(-1,0)=s_{0}^{\infty} \mathrm{e}^{\frac{\pi a}{2}}, \widehat{g}_{11}(-1,0)=-g_{22} \mathrm{e}^{\frac{3 \pi a}{4}}, \widehat{g}_{12}(-1,0)=-\left(g_{21}+\right.$ $\left.s_{0}^{\infty} g_{22}\right) \mathrm{e}^{-\frac{3 \pi a}{4}}, \widehat{g}_{21}(-1,0)=\left(s_{0}^{0} g_{22}-g_{12}\right) \mathrm{e}^{\frac{3 \pi a}{4}}$, and $\widehat{g}_{22}(-1,0)=\left(s_{0}^{0}\left(g_{21}+s_{0}^{\infty} g_{22}\right)-g_{11}-s_{0}^{\infty} g_{12}\right) \mathrm{e}^{-\frac{3 \pi a}{4}}$;

(2) $\widehat{\mathcal{F}}_{-1,-1}: \widehat{s}_{0}^{\infty}(-1,-1)=s_{0}^{\infty} \mathrm{e}^{-\frac{\pi a}{2}}, \widehat{s}_{1}^{\infty}(-1,-1)=s_{1}^{\infty} \mathrm{e}^{\frac{\pi a}{2}}, \widehat{g}_{11}(-1,-1)=-\mathrm{i} g_{21} \mathrm{e}^{-\frac{\pi a}{4}}, \widehat{g}_{12}(-1,-1)=$ $-\mathrm{i} g_{22} \mathrm{e}^{\frac{\pi a}{4}}, \widehat{g}_{21}(-1,-1)=-\mathrm{i}\left(g_{11}-s_{0}^{0} g_{21}\right) \mathrm{e}^{-\frac{\pi a}{4}}$, and $\widehat{g}_{22}(-1,-1)=-\mathrm{i}\left(g_{12}-s_{0}^{0} g_{22}\right) \mathrm{e}^{\frac{\pi a}{4}} ;$

(3) $\widehat{\mathcal{F}}_{-1,1}: \widehat{s}_{0}^{\infty}(-1,1)=s_{0}^{\infty} \mathrm{e}^{-\frac{\pi a}{2}}, \widehat{s}_{1}^{\infty}(-1,1)=s_{1}^{\infty} \mathrm{e}^{\frac{\pi a}{2}}, \widehat{g}_{11}(-1,1)=g_{11} \mathrm{e}^{-\frac{\pi a}{4}}, \widehat{g}_{12}(-1,1)=g_{12} \mathrm{e}^{\frac{\pi a}{4}}$, $\widehat{g}_{21}(-1,1)=g_{21} \mathrm{e}^{-\frac{\pi a}{4}}$, and $\widehat{g}_{22}(-1,1)=g_{22} \mathrm{e}^{\frac{\pi a}{4}}$;

(4) $\widehat{\mathcal{F}}_{1,0}: \widehat{s}_{0}^{\infty}(1,0)=s_{1}^{\infty} \mathrm{e}^{\frac{\pi a}{2}}, \widehat{s}_{1}^{\infty}(1,0)=s_{0}^{\infty} \mathrm{e}^{\frac{3 \pi a}{2}}, \widehat{g}_{11}(1,0)=-\mathrm{i} g_{12} \mathrm{e}^{\frac{\pi a}{4}}, \widehat{g}_{12}(1,0)=-\mathrm{i}\left(g_{11}+s_{0}^{\infty} g_{12}\right) \mathrm{e}^{-\frac{\pi a}{4}}$, $\widehat{g}_{21}(1,0)=-\mathrm{i} g_{22} \mathrm{e}^{\frac{1 \pi a}{4}}$, and $\widehat{g}_{22}(1,0)=-\mathrm{i}\left(g_{21}+s_{0}^{\infty} g_{22}\right) \mathrm{e}^{-\frac{\pi a}{4}}$;

\footnotetext{
${ }^{18}$ There are a few innocuous misprints in 11, which do not, however, affect the final results: (i) the factor $3 \sqrt{3}-2$ appearing on the right-hand sides of Equations (76) and (92) should read $3(\sqrt{3}-1)$; (ii) the factor $\mathrm{i}(\varepsilon b)^{1 / 3} \tau^{2 / 3}$ appearing on the right-hand side of Equation (98) and in the argument of the exponential function on the right-hand side of Equation (101) should read $\frac{3 \mathrm{i}}{2}(\varepsilon b)^{1 / 3} \tau^{2 / 3}$; and (iii) the factor $\mathrm{i}(3 \sqrt{3}-1)(\varepsilon b)^{1 / 3} \tau^{2 / 3}$ appearing on the right-hand side of Equation (99) should read $\mathrm{i}\left(3 \sqrt{3}-\frac{3}{2}\right)(\varepsilon b)^{1 / 3} \tau^{2 / 3}$.

${ }^{19}$ In transformation $6.2 .2, \tau \rightarrow \tau$, that is, $\tau_{n}=\tau_{o}$.

${ }^{20}$ In transformation $6.2 .3, a \rightarrow a$, that is, $a_{n}=a_{o}$.

${ }^{21}$ There is a misprint on page 1202 (Appendix) of [1]: for items (1) and (4) in the definition of the auxiliary mapping $\widehat{\mathcal{F}}_{\varepsilon_{1}, \varepsilon_{2}}$, the change $a \rightarrow-a$ should be made everywhere.

${ }^{22} s_{0}^{0}\left(\varepsilon_{1}, \varepsilon_{2}\right)=s_{0}^{0}$.
} 
(5) $\widehat{\mathcal{F}}_{1,-1}: \widehat{s}_{0}^{\infty}(1,-1)=s_{0}^{\infty} \mathrm{e}^{\frac{\pi a}{2}}, \widehat{s}_{1}^{\infty}(1,-1)=s_{1}^{\infty} \mathrm{e}^{-\frac{\pi a}{2}}, \widehat{g}_{11}(1,-1)=g_{11} \mathrm{e}^{\frac{\pi a}{4}}, \widehat{g}_{12}(1,-1)=g_{12} \mathrm{e}^{-\frac{\pi a}{4}}$, $\widehat{g}_{21}(1,-1)=g_{21} \mathrm{e}^{\frac{\pi a}{4}}$, and $\widehat{g}_{22}(1,-1)=g_{22} \mathrm{e}^{-\frac{\pi a}{4}}$;

(6) $\widehat{\mathcal{F}}_{1,1}: \widehat{s}_{0}^{\infty}(1,1)=s_{0}^{\infty} \mathrm{e}^{\frac{\pi a}{2}}, \widehat{s}_{1}^{\infty}(1,1)=s_{1}^{\infty} \mathrm{e}^{-\frac{\pi a}{2}}, \widehat{g}_{11}(1,1)=\mathrm{i}\left(g_{21}+s_{0}^{0} g_{11}\right) \mathrm{e}^{\frac{\pi a}{4}}, \widehat{g}_{12}(1,1)=\mathrm{i}\left(g_{22}+\right.$ $\left.s_{0}^{0} g_{12}\right) \mathrm{e}^{-\frac{\pi a}{4}}, \widehat{g}_{21}(1,1)=\mathrm{i} g_{11} \mathrm{e}^{\frac{\pi a}{4}}$, and $\widehat{g}_{22}(1,1)=\mathrm{i} g_{12} \mathrm{e}^{-\frac{\pi a}{4}}$.

Theorem C.1. Let $\varepsilon_{1}= \pm 1, \varepsilon_{2}=0, \pm 1, \epsilon b=|\epsilon b| \mathrm{e}^{\mathrm{i} \pi \varepsilon_{2}}$, and $u(\tau)$ be a solution of Equation (1.1) corresponding to the monodromy data $\left(a, s_{0}^{0}, s_{0}^{\infty}, s_{1}^{\infty}, g_{11}, g_{12}, g_{21}, g_{22}\right)$. Suppose that

$$
\widehat{g}_{11}\left(\varepsilon_{1}, \varepsilon_{2}\right) \widehat{g}_{12}\left(\varepsilon_{1}, \varepsilon_{2}\right) \widehat{g}_{21}\left(\varepsilon_{1}, \varepsilon_{2}\right) \widehat{g}_{22}\left(\varepsilon_{1}, \varepsilon_{2}\right) \neq 0, \quad \operatorname{Re}\left(\widehat{\nu}\left(\varepsilon_{1}, \varepsilon_{2}\right)+1\right) \in(0,1) \backslash\left\{\frac{1}{2}\right\}
$$

where

$$
\widehat{\nu}\left(\varepsilon_{1}, \varepsilon_{2}\right)+1:=\frac{\mathrm{i}}{2 \pi} \ln \left(\widehat{g}_{11}\left(\varepsilon_{1}, \varepsilon_{2}\right) \widehat{g}_{22}\left(\varepsilon_{1}, \varepsilon_{2}\right)\right) .
$$

Then there exist $\delta_{G}$ satisfying, for $0<\operatorname{Re}\left(\widehat{\nu}\left(\varepsilon_{1}, \varepsilon_{2}\right)+1\right)<\frac{1}{2}$, the inequality

$$
0<\delta_{G}<\frac{1}{3}\left(\frac{1+2 \operatorname{Re}\left(\widehat{\nu}\left(\varepsilon_{1}, \varepsilon_{2}\right)+1\right)}{7+6 \operatorname{Re}\left(\widehat{\nu}\left(\varepsilon_{1}, \varepsilon_{2}\right)+1\right)}\right),
$$

and, for $\frac{1}{2}<\operatorname{Re}\left(\widehat{\nu}\left(\varepsilon_{1}, \varepsilon_{2}\right)+1\right)<1$, the inequality

$$
0<\delta_{G}<\frac{1}{3}\left(\frac{3-2 \operatorname{Re}\left(\widehat{\nu}\left(\varepsilon_{1}, \varepsilon_{2}\right)+1\right)}{9+2 \operatorname{Re}\left(\widehat{\nu}\left(\varepsilon_{1}, \varepsilon_{2}\right)+1\right)}\right),
$$

such that $u(\tau)$ has the asymptotic expansion

$$
\begin{aligned}
u(\tau) \underset{\tau \rightarrow \infty}{ }=\mathrm{e}^{\frac{\mathrm{i} \pi \varepsilon_{1}}{2}} \frac{\mathrm{e}^{-\frac{\mathrm{i} \pi \varepsilon_{1}}{2}} \epsilon(\epsilon b)^{2 / 3}}{2}\left(\mathrm{e}^{-\frac{\mathrm{i} \pi \varepsilon_{1}}{2}} \tau\right)^{1 / 3}\left(1-\frac{3}{2 \sin ^{2}\left(\frac{1}{2} \beta\left(\varepsilon_{1}, \varepsilon_{2}, \tau\right)\right)}\right) \\
\quad=\underset{\tau \rightarrow \infty \mathrm{e}^{\frac{\mathrm{i} \pi \varepsilon_{1}}{2}}}{ } \frac{\mathrm{e}^{-\frac{\mathrm{i} \pi \varepsilon_{1}}{2}} \epsilon(\epsilon b)^{2 / 3}}{2}\left(\mathrm{e}^{-\frac{\mathrm{i} \pi \varepsilon_{1}}{2}} \tau\right)^{1 / 3} \frac{\sin \left(\frac{1}{2} \beta\left(\varepsilon_{1}, \varepsilon_{2}, \tau\right)-\vartheta_{0}\right) \sin \left(\frac{1}{2} \beta\left(\varepsilon_{1}, \varepsilon_{2}, \tau\right)+\vartheta_{0}\right)}{\sin ^{2}\left(\frac{1}{2} \beta\left(\varepsilon_{1}, \varepsilon_{2}, \tau\right)\right)},
\end{aligned}
$$

where

$$
\begin{aligned}
\beta\left(\varepsilon_{1}, \varepsilon_{2}, \tau\right): & =\widehat{\phi}(\tau)-\mathrm{i}\left(\left(\widehat{\nu}\left(\varepsilon_{1}, \varepsilon_{2}\right)+1\right)-\frac{1}{2}\right) \ln \widehat{\phi}(\tau)-\mathrm{i}\left(\left(\widehat{\nu}\left(\varepsilon_{1}, \varepsilon_{2}\right)+1\right)-\frac{1}{2}\right) \ln 12+(-1)^{1+\varepsilon_{2}} a \ln (2+\sqrt{3}) \\
& +\frac{\pi}{4}-\frac{3 \pi}{2}\left(\widehat{\nu}\left(\varepsilon_{1}, \varepsilon_{2}\right)+1\right)+\mathrm{i} \ln \left(\frac{\widehat{g}_{11}\left(\varepsilon_{1}, \varepsilon_{2}\right) \widehat{g}_{12}\left(\varepsilon_{1}, \varepsilon_{2}\right) \Gamma\left(\widehat{\nu}\left(\varepsilon_{1}, \varepsilon_{2}\right)+1\right)}{\sqrt{2 \pi}}\right)+\mathcal{O}\left(\tau^{-\delta_{G}} \ln \tau\right), \quad \text { (C.5) }
\end{aligned}
$$

with

$$
\widehat{\phi}(\tau)=3 \sqrt{3}(-1)^{\varepsilon_{2}}(\epsilon b)^{1 / 3}\left(\mathrm{e}^{-\frac{\mathrm{i} \pi \varepsilon_{1}}{2}} \tau\right)^{2 / 3},
$$

$\vartheta_{0}$ given in Equation (2.7), and $\Gamma(\cdot)$ the Euler gamma function [11.

Let $\mathcal{H}(\tau)$ be the Hamiltonian function defined by Equation (1.2) corresponding to the function $u(\tau)$ given above. Then $\mathcal{H}(\tau)$ has the asymptotic expansion

$$
\begin{aligned}
\mathcal{H}(\tau) \underset{\tau \rightarrow \infty \mathrm{e}^{\frac{\mathrm{i} \pi \varepsilon_{1}}{2}}}{ } \mathrm{e}^{-\frac{\mathrm{i} \pi \varepsilon_{1}}{2}}\left(3(\epsilon b)^{2 / 3}\left(\mathrm{e}^{-\frac{\mathrm{i} \pi \varepsilon_{1}}{2}} \tau\right)^{1 / 3}-\mathrm{i}(-1)^{\varepsilon_{2}} 4 \sqrt{3}(\epsilon b)^{1 / 3}\left(\mathrm{e}^{-\frac{\mathrm{i} \pi \varepsilon_{1}}{2}} \tau\right)^{-1 / 3}\left(\left(\widehat{\nu}\left(\varepsilon_{1}, \varepsilon_{2}\right)+1\right)\right.\right. \\
\quad-\frac{1}{2}+\frac{1}{2 \sqrt{3}}\left(\mathrm{i}(-1)^{1+\varepsilon_{2}} a+\frac{1}{2}\right)+\frac{\mathrm{i}}{4} \cot \left(\frac{1}{2} \beta\left(\varepsilon_{1}, \varepsilon_{2}, \tau\right)\right)+\frac{\mathrm{i}}{4} \cot \left(\frac{1}{2} \beta\left(\varepsilon_{1}, \varepsilon_{2}, \tau\right)-\vartheta_{0}\right) \\
+ \\
\left.\left.+\mathcal{O}\left(\tau^{-\delta_{G}}\right)\right)\right) .
\end{aligned}
$$

The function $f(\tau)$ defined by Equation (1.3) has the following asymptotics:

$$
f(\tau) \underset{\tau \rightarrow \infty \mathrm{e}^{\frac{\mathrm{i} \pi \varepsilon_{1}}{2}}}{=}-\frac{(-1)^{\varepsilon_{2}}(\epsilon b)^{1 / 3}}{2}\left(\mathrm{e}^{-\frac{\mathrm{i} \pi \varepsilon_{1}}{2}} \tau\right)^{2 / 3}\left(\mathrm{i}+\frac{3}{\sqrt{2} \sin \left(\frac{1}{2} \beta\left(\varepsilon_{1}, \varepsilon_{2}, \tau\right)\right) \sin \left(\frac{1}{2} \beta\left(\varepsilon_{1}, \varepsilon_{2}, \tau\right)-\vartheta_{0}\right)}\right) .
$$


Remark C.1. Define the strip (in the $\widehat{\phi}$-plane)

$$
\widehat{\mathcal{D}}:=\left\{\tau \in \mathbb{C}: \operatorname{Re}(\widehat{\phi}(\tau))>c_{1},|\operatorname{Im}(\widehat{\phi}(\tau))|<c_{2}\right\},
$$

where $\widehat{\phi}(\tau)$ is given in Equation (C.6), and $c_{1}, c_{2}>0$ are parameters. The asymptotics of $u(\tau), \mathcal{H}(\tau)$, and $f(\tau)$ presented in Theorem C.1 are actually valid in the strip domain $\widehat{\mathcal{D}}$.

Theorem C.2. Let $\varepsilon_{1}= \pm 1, \varepsilon_{2}=0, \pm 1, \epsilon b=|\epsilon b| \mathrm{e}^{\mathrm{i} \pi \varepsilon_{2}}$, and $u(\tau)$ be a solution of Equation (1.1) corresponding to the monodromy data $\left(a, s_{0}^{0}, s_{0}^{\infty}, s_{1}^{\infty}, g_{11}, g_{12}, g_{21}, g_{22}\right)$. Suppose that

$$
\widehat{g}_{11}\left(\varepsilon_{1}, \varepsilon_{2}\right) \widehat{g}_{12}\left(\varepsilon_{1}, \varepsilon_{2}\right) \widehat{g}_{21}\left(\varepsilon_{1}, \varepsilon_{2}\right) \widehat{g}_{22}\left(\varepsilon_{1}, \varepsilon_{2}\right) \neq 0, \quad \operatorname{Re}\left(\frac{\mathrm{i}}{2 \pi} \ln \left(\widehat{g}_{11}\left(\varepsilon_{1}, \varepsilon_{2}\right) \widehat{g}_{22}\left(\varepsilon_{1}, \varepsilon_{2}\right)\right)\right)=\frac{1}{2} .
$$

Let the branch of the function $\ln (\cdot)$ be chosen ${ }^{23}$ such that $\operatorname{Im}\left(\ln \left(-\widehat{g}_{11}\left(\varepsilon_{1}, \varepsilon_{2}\right) \widehat{g}_{22}\left(\varepsilon_{1}, \varepsilon_{2}\right)\right)\right)=0$. Define

$$
\widehat{\varrho}_{1}\left(\varepsilon_{1}, \varepsilon_{2}\right):=\frac{1}{2 \pi} \ln \left(-\widehat{g}_{11}\left(\varepsilon_{1}, \varepsilon_{2}\right) \widehat{g}_{22}\left(\varepsilon_{1}, \varepsilon_{2}\right)\right) \quad(\in \mathbb{R}) .
$$

Then $\exists \delta \in(0,1 / 39)$ such that the function $u(\tau)$ has, for all large enough $m \in \mathbb{N}$, second-order poles, $\widehat{\tau}_{m}^{\infty}$, accumulating at the point at infinity,

$$
\left.\widehat{\tau}_{m}^{\infty} \underset{m \rightarrow \infty}{=} \mathrm{e}^{\mathrm{i} \pi \varepsilon_{1}} \frac{2 \pi(-1)^{\varepsilon_{2}} m}{3 \sqrt{3}(\epsilon b)^{1 / 3}}\right)^{3 / 2}\left(1-\frac{3 \widehat{\varrho}_{1}\left(\varepsilon_{1}, \varepsilon_{2}\right)}{4 \pi} \frac{\ln m}{m}-\frac{3 \widehat{\varrho}_{2}\left(\varepsilon_{1}, \varepsilon_{2}\right)}{4 \pi} \frac{1}{m}\right)+\mathcal{O}\left(m^{\frac{1}{2}-\frac{3 \delta}{2}}\right),
$$

where

$$
\begin{aligned}
\widehat{\varrho}_{2}\left(\varepsilon_{1}, \varepsilon_{2}\right) & :=\widehat{\varrho}_{1}\left(\varepsilon_{1}, \varepsilon_{2}\right) \ln (24 \pi)+(-1)^{1+\varepsilon_{2}} \operatorname{Re}(a) \ln (2+\sqrt{3})+\frac{\pi}{2}-\frac{1}{2} \arg \left(\frac{\widehat{g}_{11}\left(\varepsilon_{1}, \varepsilon_{2}\right) \widehat{g}_{12}\left(\varepsilon_{1}, \varepsilon_{2}\right)}{\widehat{g}_{21}\left(\varepsilon_{1}, \varepsilon_{2}\right) \widehat{g}_{22}\left(\varepsilon_{1}, \varepsilon_{2}\right)}\right) \\
& -\arg \left(\Gamma\left(\frac{1}{2}+\mathrm{i} \widehat{\varrho}_{1}\left(\varepsilon_{1}, \varepsilon_{2}\right)\right)\right)+\mathrm{i}\left((-1)^{1+\varepsilon_{2}} \operatorname{Im}(a) \ln (2+\sqrt{3})+\frac{1}{2} \ln \left(\left|\frac{\widehat{g}_{11}\left(\varepsilon_{1}, \varepsilon_{2}\right) \widehat{g}_{12}\left(\varepsilon_{1}, \varepsilon_{2}\right)}{\widehat{g}_{21}\left(\varepsilon_{1}, \varepsilon_{2}\right) \widehat{g}_{22}\left(\varepsilon_{1}, \varepsilon_{2}\right)}\right|\right)\right) ;
\end{aligned}
$$

furthermore, the function $u(\tau)$ has, for all large enough $m \in \mathbb{N}$, a pair of first-order zeroes, $\widehat{\tau}_{m}^{ \pm}$, accumulating at the point at infinity,

$$
\widehat{\tau}_{m}^{ \pm} \underset{m \rightarrow \infty}{=} \mathrm{e}^{\frac{\mathrm{i} \pi \varepsilon_{1}}{2}}\left(\frac{2 \pi(-1)^{\varepsilon_{2}} m}{3 \sqrt{3}(\epsilon b)^{1 / 3}}\right)^{3 / 2}\left(1-\frac{3 \widehat{\varrho}_{1}\left(\varepsilon_{1}, \varepsilon_{2}\right)}{4 \pi} \frac{\ln m}{m}-\frac{3}{4 \pi}\left(\varrho_{2}\left(\varepsilon_{1}, \varepsilon_{2}\right) \pm 2 \vartheta_{0}\right) \frac{1}{m}\right)+\mathcal{O}\left(m^{\frac{1}{2}-\frac{3 \delta}{2}}\right),
$$

where $\vartheta_{0}$ given in Equation (2.7).

Remark C.2. To present asymptotics of $u(\tau), \mathcal{H}(\tau)$, and $f(\tau)$ outside of neighborhoods of poles and zeroes, introduce the cheese-like domain, $\widehat{\mathcal{D}}_{u}$, for a solution $u(\tau)$ :

$$
\widehat{\mathcal{D}}_{u}:=\left\{\tau \in \widehat{\mathcal{D}}:\left|\widehat{\phi}(\tau)-\widehat{\phi}\left(\widehat{\tau}_{m}^{\kappa}\right)\right| \geqslant C\left|\widehat{\tau}_{m}^{\kappa}\right|^{-\delta}\right\},
$$

where the strip domain $\widehat{\mathcal{D}}$ is defined by Equation (C.9), $\widehat{\phi}(\tau)$ is given in Equation (C.6), $C>0$ is a parameter, $\kappa=\infty, \pm\left(\widehat{\tau}_{m}^{\kappa}\right.$ are the poles and zeroes introduced in Theorem C.2 $)$, and $0<\delta<1 / 39$.

Theorem C.3. Let $\varepsilon_{1}= \pm 1, \varepsilon_{2}=0, \pm 1, \epsilon b=|\epsilon b| \mathrm{e}^{\mathrm{i} \pi \varepsilon_{2}}$, and $u(\tau)$ be a solution of Equation (1.1) corresponding to the monodromy data $\left(a, s_{0}^{0}, s_{0}^{\infty}, s_{1}^{\infty}, g_{11}, g_{12}, g_{21}, g_{22}\right)$. Suppose that conditions (C.10) are valid, the branch of $\ln (\cdot)$ is chosen as in Theorem [C.2, and $\widehat{\varrho}_{1}\left(\varepsilon_{1}, \varepsilon_{2}\right)$ is defined by Equation (C.11). Then there exist $\delta, \delta_{G} \in \mathbb{R}_{+}$satisfying the inequalities

$$
0<\delta<\frac{1}{39}, \quad 0<\delta<\delta_{G}<\frac{1}{15}-\frac{8 \delta}{5},
$$

such that $u(\tau)$ has the asymptotic expansion

$$
u(\tau) \underset{\substack{\tau \rightarrow \infty \infty \\ \tau \in \widehat{\mathcal{D}}_{u}}}{=} \underbrace{}_{\substack{\mathrm{i} \varepsilon_{1} \\ \tau \in}} \frac{\mathrm{e}^{-\frac{\mathrm{i} \pi \varepsilon_{1}}{2}} \epsilon(\epsilon b)^{2 / 3}}{2}\left(\mathrm{e}^{-\frac{\mathrm{i} \pi \varepsilon_{1}}{2}} \tau\right)^{1 / 3} \frac{\sin \left(\frac{1}{2} \widehat{\beta}\left(\varepsilon_{1}, \varepsilon_{2}, \tau\right)-\vartheta_{0}\right) \sin \left(\frac{1}{2} \widehat{\beta}\left(\varepsilon_{1}, \varepsilon_{2}, \tau\right)+\vartheta_{0}\right)}{\sin ^{2}\left(\frac{1}{2} \widehat{\beta}\left(\varepsilon_{1}, \varepsilon_{2}, \tau\right)\right)},
$$

${ }^{23}$ The second condition of Equations (C.10) suggests that this branch of $\ln (\cdot)$ exists. 
where

$$
\begin{aligned}
\widehat{\beta}\left(\varepsilon_{1}, \varepsilon_{2}, \tau\right): & =\widehat{\phi}(\tau)+\widehat{\varrho}_{1}\left(\varepsilon_{1}, \varepsilon_{2}\right) \ln \widehat{\phi}(\tau)+\widehat{\varrho}_{1}\left(\varepsilon_{1}, \varepsilon_{2}\right) \ln 12+(-1)^{1+\varepsilon_{2}} \operatorname{Re}(a) \ln (2+\sqrt{3})+\frac{\pi}{2} \\
& -\frac{1}{2} \arg \left(\frac{\widehat{g}_{11}\left(\varepsilon_{1}, \varepsilon_{2}\right) \widehat{g}_{12}\left(\varepsilon_{1}, \varepsilon_{2}\right)}{\widehat{g}_{21}\left(\varepsilon_{1}, \varepsilon_{2}\right) \widehat{g}_{22}\left(\varepsilon_{1}, \varepsilon_{2}\right)}\right)-\arg \left(\Gamma\left(\frac{1}{2}+\mathrm{i} \widehat{\varrho}_{1}\left(\varepsilon_{1}, \varepsilon_{2}\right)\right)\right)+\mathrm{i}\left((-1)^{1+\varepsilon_{2}} \operatorname{Im}(a) \ln (2+\sqrt{3})\right. \\
& \left.+\frac{1}{2} \ln \left(\left|\frac{\widehat{g}_{11}\left(\varepsilon_{1}, \varepsilon_{2}\right) \widehat{g}_{12}\left(\varepsilon_{1}, \varepsilon_{2}\right)}{\widehat{g}_{21}\left(\varepsilon_{1}, \varepsilon_{2}\right) \widehat{g}_{22}\left(\varepsilon_{1}, \varepsilon_{2}\right)}\right|\right)\right)+\mathcal{O}\left(\tau^{-\delta_{G}} \ln \tau\right),
\end{aligned}
$$

with $\widehat{\phi}(\tau)$ and $\vartheta_{0}$ given, respectively, in Equations (C.6) and (2.7).

Let $\mathcal{H}(\tau)$ be the Hamiltonian function defined by Equation (1.2) corresponding to the function $u(\tau)$ given above. Then $\mathcal{H}(\tau)$ has the asymptotic expansion

$$
\begin{aligned}
& \mathcal{H}(\tau) \underset{\substack{\tau \rightarrow \infty \\
\tau \in \mathrm{e}_{u} \frac{\mathrm{i} \pi \varepsilon_{1}}{2}}}{=} \mathrm{e}^{-\frac{\mathrm{i} \pi \varepsilon_{1}}{2}}\left(3(\epsilon b)^{2 / 3}\left(\mathrm{e}^{-\frac{\mathrm{i} \pi \varepsilon_{1}}{2}} \tau\right)^{1 / 3}+(-1)^{\varepsilon_{2}} 4 \sqrt{3}(\epsilon b)^{1 / 3}\left(\mathrm{e}^{-\frac{\mathrm{i} \pi \varepsilon_{1}}{2}} \tau\right)^{-1 / 3}\left(\widehat{\varrho}_{1}\left(\varepsilon_{1}, \varepsilon_{2}\right)\right.\right. \\
& +\frac{1}{2 \sqrt{3}}\left((-1)^{1+\varepsilon_{2}} a-\frac{\mathrm{i}}{2}\right)+\frac{1}{4} \cot \left(\frac{1}{2} \widehat{\beta}\left(\varepsilon_{1}, \varepsilon_{2}, \tau\right)\right)+\frac{1}{4} \cot \left(\frac{1}{2} \widehat{\beta}\left(\varepsilon_{1}, \varepsilon_{2}, \tau\right)-\vartheta_{0}\right) \\
& \left.\left.+\mathcal{O}\left(\tau^{-\delta_{G}}\right)\right)\right) \text {. }
\end{aligned}
$$

The function $f(\tau)$ defined by Equation (1.3) has the following asymptotics:

$$
f(\tau) \underset{\substack{\tau \rightarrow \infty \mathrm{e}^{\mathrm{i} \pi \varepsilon_{1}} \\ \tau \in \widehat{\mathcal{D}}_{u}}}{=}-\frac{(-1)^{\varepsilon_{2}}(\epsilon b)^{1 / 3}}{2}\left(\mathrm{e}^{-\frac{\mathrm{i} \pi \varepsilon_{1}}{2}} \tau\right)^{2 / 3}\left(\mathrm{i}+\frac{3}{\sqrt{2} \sin \left(\frac{1}{2} \widehat{\beta}\left(\varepsilon_{1}, \varepsilon_{2}, \tau\right)\right) \sin \left(\frac{1}{2} \widehat{\beta}\left(\varepsilon_{1}, \varepsilon_{2}, \tau\right)-\vartheta_{0}\right)}\right) .
$$

Remark C.3. For real, non-zero values of $b$, singular imaginary solutions $u(\tau)$ (for imaginary $\tau$ ) of Equation (1.1) are specified by the following "singular imaginary reduction" for the monodromy dat.24:

$$
\begin{array}{cc}
s_{0}^{0}=-\overline{s_{0}^{0}}, \quad \widehat{s}_{0}^{\infty}\left(\varepsilon_{1}, \varepsilon_{2}\right)=-\overline{\widehat{s}_{1}^{\infty}\left(\varepsilon_{1}, \varepsilon_{2}\right)} \mathrm{e}^{2 \pi a}, & \widehat{g}_{11}\left(\varepsilon_{1}, \varepsilon_{2}\right)=-\overline{\widehat{g}_{22}\left(\varepsilon_{1}, \varepsilon_{2}\right)}, \\
\widehat{g}_{12}\left(\varepsilon_{1}, \varepsilon_{2}\right)=-\overline{\widehat{g}_{21}\left(\varepsilon_{1}, \varepsilon_{2}\right)}, & \operatorname{Im}(a)=0 .
\end{array}
$$

In this case, asymptotics of $\widehat{\tau}_{m}^{\infty}, \widehat{\tau}_{m}^{ \pm}, u(\tau), \mathcal{H}(\tau)$, and $f(\tau)$ are as given in Equations (C.12), (C.14), (C.15), (C.17), and (C.18), respectively, but with the changes $\widehat{\varrho}_{1}\left(\varepsilon_{1}, \varepsilon_{2}\right) \rightarrow \widehat{\varrho}_{0}\left(\varepsilon_{1}, \varepsilon_{2}\right), \widehat{\varrho}_{2}\left(\varepsilon_{1}, \varepsilon_{2}\right) \rightarrow$ $\widehat{\varrho}_{0}^{\sharp}\left(\varepsilon_{1}, \varepsilon_{2}\right)$, and $\widehat{\beta}\left(\varepsilon_{1}, \varepsilon_{2}, \tau\right) \rightarrow \widehat{\beta}_{0}\left(\varepsilon_{1}, \varepsilon_{2}, \tau\right)$, where

$$
\begin{aligned}
\widehat{\varrho}_{0}\left(\varepsilon_{1}, \varepsilon_{2}\right) & :=\frac{1}{\pi} \ln \left|\widehat{g}_{11}\left(\varepsilon_{1}, \varepsilon_{2}\right)\right|, \\
\widehat{\varrho}_{0}^{\sharp}\left(\varepsilon_{1}, \varepsilon_{2}\right): & =\widehat{\varrho}_{0}\left(\varepsilon_{1}, \varepsilon_{2}\right) \ln (24 \pi)+(-1)^{1+\varepsilon_{2}} \operatorname{Re}(a) \ln (2+\sqrt{3})-\frac{\pi}{2} \\
& -\arg \left(\widehat{g}_{11}\left(\varepsilon_{1}, \varepsilon_{2}\right) \widehat{g}_{12}\left(\varepsilon_{1}, \varepsilon_{2}\right) \Gamma\left(\frac{1}{2}+\mathrm{i} \widehat{\varrho}_{0}\left(\varepsilon_{1}, \varepsilon_{2}\right)\right)\right), \\
\widehat{\beta}_{0}\left(\varepsilon_{1}, \varepsilon_{2}, \tau\right): & =\widehat{\phi}(\tau)+\widehat{\varrho}_{0}\left(\varepsilon_{1}, \varepsilon_{2}\right) \ln \widehat{\phi}(\tau)+\widehat{\varrho}_{0}\left(\varepsilon_{1}, \varepsilon_{2}\right) \ln 12+(-1)^{1+\varepsilon_{2}} \operatorname{Re}(a) \ln (2+\sqrt{3}) \\
& -\frac{\pi}{2}-\arg \left(\widehat{g}_{11}\left(\varepsilon_{1}, \varepsilon_{2}\right) \widehat{g}_{12}\left(\varepsilon_{1}, \varepsilon_{2}\right) \Gamma\left(\frac{1}{2}+\mathrm{i} \widehat{\varrho}_{0}\left(\varepsilon_{1}, \varepsilon_{2}\right)\right)\right)+\mathcal{O}\left(\tau^{-\delta_{G}} \ln \tau\right) .
\end{aligned}
$$

\footnotetext{
${ }^{24}$ There exist regular imaginary solutions (cf. Part I [1], Appendix) which are specified by another imaginary reduction.
} 


\section{Bibliography}

[1] A. V. Kitaev and A. H. Vartanian, "Connection formulae for asymptotics of solutions of the degenerate third Painlevé equation: I", Inverse Problems, Vol. 20, No. 4, pp. 1165-1206, 2004.

[2] A. R. Its and V. Yu. Novokshenov, The Isomonodromic Deformation Method in the Theory of Painlevé Equations, Lecture Notes in Mathematics, Vol. 1191, Springer-Verlag, Berlin, 1986.

[3] Y. Ohyama, H. Kawamuko, H. Sakai, and K. Okamoto, "Studies on the Painlevé equations. V. Third Painlevé equations of special type $P_{\mathrm{III}}\left(D_{7}\right)$ and $P_{\mathrm{III}}\left(D_{8}\right)$ ", J. Math. Sci. Univ. Tokyo, Vol. 13, No. 2, pp. 145-204, 2006.

[4] H. Wakako and Y. Takei, "Exact WKB analysis for the degenerate third Painlevé equation of type $\left(D_{8}\right)$ ", Proc. Japan Acad. Ser. A Math. Sci., Vol. 83, No. 5, pp. 63-68, 2007.

[5] M. Dunajski and P. Plansangkate, "Strominger-Yau-Zaslow Geometry, Affine Spheres and Painlevé III", Comm. Math. Phys., Vol. 290, No. 3, pp. 997-1024, 2009.

[6] A. S. Fokas, A. R. Its, A. A. Kapaev, and V. Yu. Novokshenov, Painlevé Transcendents. The Riemann-Hilbert Approach, Mathematical Surveys and Monographs, Vol. 128, AMS, Providence, 2006.

[7] P. A. Deift and X. Zhou, "Asymptotics for the Painlevé II Equation", Comm. Pure Appl. Math., Vol. 48, No. 3, pp. 277-337, 1995.

[8] W. Wasow, Linear Turning Point Theory, Applied Mathematical Sciences, Vol. 54, SpringerVerlag, New York, 1985.

[9] M. V. Fedoryuk, Asymptotic Analysis, Springer-Verlag, New York, 1993.

[10] A. V. Kitaev, "The justification of asymptotic formulas that can be obtained by the method of isomonodromic deformations", Zap. Nauchn. Sem. Leningrad Otdel. Mat. Inst. Steklov. (LOMI), Vol. 179, pp. 101-109, 1989 (Engl. Transl.: J. Soviet Math., Vol. 57, No. 3, pp. 3131$3135,1991)$.

[11] I. S. Gradshteyn and I. M. Ryzhik, 5th edn., Tables of Integrals, Series, and Products, A. Jeffrey, ed., Academic Press, San Diego, 1994.

[12] A. R. Its, "Isomonodromic solutions of equations of zero curvature", Izv. Akad. Nauk SSSR Ser. Mat., Vol. 49, No. 3, pp. 530-565, 1985 (Engl. Transl.: Math. USSR-Izv., Vol. 26, No. 3, pp 497-529, 1986).

[13] A. Erdélyi, W. Magnus, F. Oberhettinger, and F. G. Tricomi, Higher Transcendental Functions, Vol. II, Robert E. Krieger Publishing Co., Inc., Melbourne, Fla., 1981.

[14] A. R. Its, A. S. Fokas, and A. A. Kapaev, "On the asymptotic analysis of the Painlevé equations via the isomonodromy method", Nonlinearity, Vol. 7, No. 5, pp. 1291-1325, 1994. 